\title{
Thetafunktionen und konjugationsinvariante Funktionen auf Paaren von Matrizen
}

\author{
Dissertation \\ zur Erlangung des Doktorgrades \\ der Mathematisch-Naturwissenschaftlichen Fakultäten \\ der Georg-August-Universität zu Göttingen
}

vorgelegt von

Annika Eickhoff-Schachtebeck

aus Bremen

Göttingen 2008 
D7

Referent: Prof. Dr. Viktor Pidstrygach

Koreferent: Prof. Dr. Ulrich Stuhler

Tag der mündlichen Prüfung: 30. September 2008 


\section{Inhaltsverzeichnis}

$\begin{array}{ll}\text { Einleitung } & 5\end{array}$

1 Allgemeines 11

1.1 Rationale Regelflächen . . . . . . . . . . . . . . . . . . . . 11

1.2 Die Spektralkurve in $\widehat{R} \ldots \ldots \ldots \ldots$. . . . . . . . . . . . . . . . . . . . . . . . . . . . 15

1.3 Thetafunktionen . . . . . . . . . . . . . . . . . 16

1.4 Der Satz von Beauville . . . . . . . . . . . . . . . . . 20

1.5 Ein fester Punkt im Thetadivisor . . . . . . . . . . . . . 21

2 Grundlegende Berechnungen zur Spektralkurve $S \quad 23$

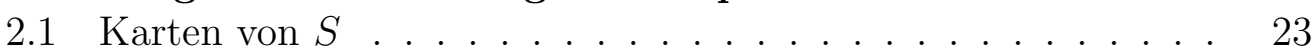

2.2 Der Tangentialraum der Jakobischen . . . . . . . . . . . . . . . 24

2.3 Beschreibung von $H^{i}(S, \mathcal{O}(n)) \ldots \ldots \ldots$

2.4 Lax-Paar-Bedingung . . . . . . . . . . . . . . . . 30

2.5 Isospektrale Flüsse durch den Thetadivisor . . . . . . . . . . . 34

3 Painlevé-Analysis $\quad 35$

3.1 Grundlegende Prinzipien . . . . . . . . . . . . . . . 36

3.2 Painlevé-Analysis . . . . . . . . . . . . . . . . . . . . . . . . . . 38

3.3 Berechnung der indizierenden Menge . . . . . . . . . . . . 43

3.4 Die Kowalevski-Matrix . . . . . . . . . . . . . . . . . . . . . . 48

3.5 Bestimmung weiterer Koeffizienten . . . . . . . . . . . 51

4 Fortsetzungen von holomorphen Schnitten $\quad 55$

4.1 Definition des Vektorbündels $V \ldots \ldots$. . . . . . . 56

4.2 Holomorphe Fortsetzungen . . . . . . . . . . . . . 57

4.3 Fortsetzungen endlicher Ordnung . . . . . . . . . . . . 58

4.4 Yoneda-Erweiterungen über $\mathbb{P}^{1} \ldots \ldots \ldots$. . . . . . . . . . . . 59

4.5 Eigenschaften von $E^{l}(n) \ldots \ldots \ldots$. . . . . . . . . 61

4.6 Kanonische Erweiterungen . . . . . . . . . . . . . . . 62

4.7 Kanonische Erweiterungen in $H^{0}\left(\mathbb{P}^{1}, E^{l}(n)\right) \ldots \ldots 7$

$4.8 E^{l}(n)$ und spezielle Fortsetzungen . . . . . . . . . . . . . 71

4.9 Yoneda-Erweiterungen über $S \ldots \ldots 73$ 
4.10 Globale Schnitte von $F^{l}(n) \ldots \ldots \ldots$. . . . . . . 75

4.11 Genaue Beschreibung von $V_{0}$. . . . . . . . . . . . . . 78

4.12 Konkrete Erweiterungen . . . . . . . . . . . . . . . . 80

5 Das asymptotische Verhalten im festen Punkt $\mathcal{O}(m) \quad 87$

5.1 Definition des Kernbündels . . . . . . . . . . . . . . . . 88

5.2 Berechnung von $K_{0} \ldots \ldots \ldots \ldots$

5.3 Erweiterungen von Elementen aus $K_{0} \ldots \ldots \ldots$. . . . . . . . 92

5.4 Berechnung der Polordnungen und Residuen . . . . . . . . . . 94

6 Beschreibung mittels Thetafunktionen $\quad 97$

$\begin{array}{lll}7 & \text { Ausblick } & 105\end{array}$ 


\section{Einleitung}

In dieser Arbeit beschäftigen wir uns mit der Darstellung konjugationsinvarianter Funktionen auf dem Raum $\mathrm{gl}_{k} \times \mathrm{gl}_{k}$ der Paare von Matrizen mittels Thetafunktionen. Dadurch stellen wir eine Verbindung zwischen zwei klassischen Gebieten der Mathematik her: den Äquivalenzklassen von Paaren von Matrizen und den Thetafunktionen.

Zwei Paare von komplexwertigen $k \times k$-Matrizen $(C, D)$ und $\left(C^{\prime}, D^{\prime}\right)$ heißen äquivalent, wenn es eine invertierbare Matrix $X \in \mathrm{GL}_{k}$ mit $X C X^{-1}=C^{\prime}$ und $X D X^{-1}=D^{\prime}$ gibt. Jede Äquivalenzklasse $(A, B) \in\left(\mathrm{gl}_{k} \times \mathrm{gl}_{k}\right) / \mathrm{GL}_{k}$ definiert eine algebraische Kurve vom Grad $k$, die sogenannte Spektralkurve, durch

$$
S_{A, B}=\left\{\left(\nu_{1}: \nu_{2}: \nu_{3}\right) \in \mathbb{P}^{2} \mid \operatorname{det}\left(\nu_{1} \mathbb{1}_{k}+\nu_{2} A+\nu_{3} B\right)=0\right\} .
$$

Die Menge $M_{S}$ aller Äquivalenzklassen von Paaren von Matrizen mit fester Spektralkurve $S$ stimmt gerade mit der affinen Jakobischen $J(S) \backslash \Theta$ überein ([E03]). Jede holomorphe Funktion $f: M_{S} \rightarrow \mathbb{C}$ entspricht folglich einer meromorphen Funktion $\widehat{f}: J(S) \rightarrow \mathbb{C}$, wobei die Polstellen von $\widehat{f}$ nur im Thetadivisor $\Theta \subset J(S)$ auftreten:

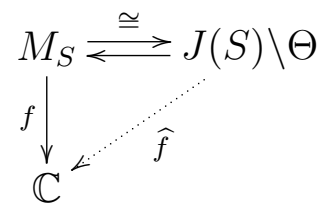

Die Menge aller meromorphen Funktionen auf $J(S)$ mit Polstellen in $\Theta$ ist bekannt. Jede solche Funktion lässt sich mittels Thetafunktionen ausdrücken ([M83]). Dadurch erhalten wir eine Verbindung zwischen Thetafunktionen und Äquivalenzklassen von Paaren von Matrizen.

Paare von Matrizen spielen in vielen Bereichen der Mathematik eine Rolle. Zum Beispiel ist die Menge aller $k$-dimensionalen Darstellungen der nicht-

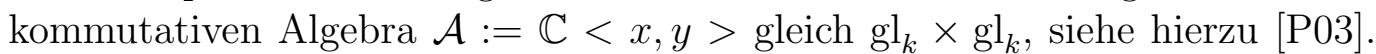
Bezeichnet $\mathcal{A}_{2}$ die Gruppe der affinen Transformationen von $\mathbb{C}^{2}$ und $\mathcal{E}$ die Gruppe der elementaren Transformationen

$$
\left\{(x, y) \mapsto\left(\lambda_{1} x+\lambda_{2}, \nu y+p(x)\right) \mid \lambda_{1}, \lambda_{2}, \nu \in \mathbb{C}, p \in \mathbb{C}[t]\right\},
$$


so wirkt das amalgamierte Produkt $\mathcal{G}:=\mathcal{A}_{2} *_{\Gamma} \mathcal{E}$ mit $\Gamma=\mathcal{A}_{2} \cap \mathcal{E}$ auf gl $\mathrm{gl}_{k} \times \mathrm{gl}_{k}$. Diese Wirkung ist durch die Wirkung der Untergruppen $\mathcal{A}_{2}$ und $\mathcal{E}$ auf $\mathrm{gl}_{k} \times \mathrm{gl}_{k}$ durch

$$
(A, B) \mapsto\left(\lambda_{1} A+\lambda_{2} B+\lambda_{3} \mathbb{1}_{k}, \nu_{1} A+\nu_{2} B+\nu 3 \mathbb{1}_{k}\right)
$$

bzw.

$$
(A, B) \mapsto\left(\lambda_{1} A+\lambda_{2} \mathbb{1}_{k}, \nu B+p(A)\right)
$$

definiert. Die Wirkung ist konjugationsinvariant und erhält bis auf skalare Vielfache den Kommutator $\mu(A, B):=[A, B]$ zweier Matrizen $A$ und $B$. Nach Artamkin ([A89], [P03]) ist der $\mathcal{G}$-Orbit $\mathcal{O}_{A, B}$ durch ein allgemeines Paar $(A, B)$ dicht in $\mu^{-1}(C)$ mit $C=[A, B]$. Sind $N_{A, B}:=\mathcal{O}_{A, B} / \mathrm{GL}_{k}$ und $N_{C}:=\left\{(A, B) \in \mathrm{gl}_{k} \times \mathrm{gl}_{k} \mid \exists \lambda \in \mathbb{C}: \mu(A, B)=\lambda C\right\} / \mathrm{GL}_{k}$, so können wir den Schnitt $M_{S} \cap N_{A, B}$ beschreiben, indem wir die Mengen der auf $N_{C}$ bzw. $M_{S}$ konstanten Funktionen betrachten und diese mittels Thetafunktionen ausdrücken.

In der Theorie der vollständig integrablen Systeme ist das Zusammenspiel von Algebraischer Geometrie und Dynamischen Systemen wohlbekannt. Viele integrable Systeme (für Beispiele siehe [BBT03]) lassen sich in der Lax-Paar-Form $\dot{C}=[C, D]$ schreiben, wobei hier $C=C(z, \zeta)$ und $D=D(z, \zeta)$ Matrixpolynome in einem Spektralparameter $\zeta$ sind. Lösungen $(C, D)$ dieser Lax-PaarGleichung definieren die gleiche Spektralkurve $S_{C, D}$ und werden durch Flüsse auf der affinen Jakobischen $J\left(S_{C, D}\right) \backslash \Theta \mathrm{zu} S_{C, D}$ beschrieben.

Wir interessieren uns für Paare von Matrizen und damit für Matrixpolynome $A+\zeta B$ vom Grad eins in $\zeta$. Anders als im Fall von Lax-Paaren ist keine Differentialgleichung gegeben, deren Lösungen wir bestimmen wollen, sondern wir suchen eine Beschreibung aller Funktionen auf $M_{S}$ durch Thetafunktionen. Trotzdem können wir auch hier die Theorie der integrablen Systeme und der Painlevé-Analysis ([V08]) nutzen. Hier liegen potentielle weitere Anwendungsgebiete dieser Arbeit. Unter Verwendung der Painlevé-Analysis können wir Untervarietäten des Thetadivisors durch Familien von formalen Laurentreihen mit Koeffizienten in $\mathrm{gl}_{k} \times \mathrm{gl}_{k} / \mathrm{GL}_{k}$ ausdrücken. Mithilfe dieser Arbeit ergibt sich dadurch unter Umständen ein neuer Zugang zur BrillNoether-Theorie, in der es darum geht, Untervarietäten von $\operatorname{Pic}^{d}(S)$ der Art $W_{d}^{r}(S)=\left\{L \in \operatorname{Pic}^{d}(S) \mid h^{0}(S, L) \geq r+1\right\}$ zu beschreiben.

Die Arbeit ist wie folgt aufgebaut:

In Kapitel 1 fassen wir Grundlagen zusammen und legen Notationen fest. In Abschnitt 1.1 geben wir bekannte Resultate zu rationalen Regelflächen an. Diese werden in Kapitel 2 bei der Beschreibung der Kohomologieklassen $H^{i}(S, \mathcal{O}(n)), n \in \mathbb{N}$, benötigt. In Abschnitt 1.2 beschreiben wir die Spektralkurve $S$ als Nullstellenmenge $(\psi)_{0}$ eines Schnittes $\psi \in H^{0}\left(R, \pi_{R}^{*} \mathcal{O}_{\mathbb{P}^{1}}(k)\right)$ im Totalraum $R$ des Geradenbündels $\mathcal{O}_{\mathbb{P}^{1}}(1)$. Bezeichnet $\eta \in H^{0}\left(R, \pi_{R}^{*} \mathcal{O}_{\mathbb{P}^{1}}(1)\right)$ 
den tautologischen Schnitt, so ist $\psi$ von der Form

$$
\psi=\operatorname{det}\left(\eta \mathbb{1}_{k}+A+\zeta B\right)=\eta^{k}+a_{1} \eta^{k-1}+\ldots+a_{k}
$$

mit $a_{i} \in H^{0}\left(\mathbb{P}^{1}, \mathcal{O}(i)\right)$. Diese Beschreibung der Spektralkurve verwenden wir in der gesamten Arbeit. In Abschnitt 1.3 fassen wir Grundlagen über Thetafunktionen zusammen und stellen eine Beschreibung aller meromorphen Funktionen auf Jakobischen mit Polstellen im Thetadivisor vor. In Abschnitt 1.4 gehen wir auf die Identifizierung von $M_{S}$ mit $J(S) \backslash \Theta$ ein. Schließlich geben wir in Abschnitt 1.5 für $m:=\frac{1}{2}(k-1)$ mit ungeradem $k$ einen festen Punkt $\mathcal{O}(m)$ im Thetadivisor $\Theta \subset J(S)$ für eine glatte Spektralkurve $S$ an.

Im zweiten Kapitel berechnen wir Basen von $H^{1}(S, \mathcal{O})$ und $H^{0}(S, \mathcal{O}(n))$ mit $n \in \mathbb{N}$. Dazu wurden die Beweisideen vom Fall der Nahmgleichungen (siehe [Hi83]) auf Spektralkurven zu Äquivalenzklassen von Paaren von Matrizen übertragen. Außerdem beschreiben wir den Raum $H^{1}(S, \mathcal{O}(m))$. In Abschnitt 2.4 gehen wir auf die wohlbekannten Lax-Paar-Bedingungen an Äquivalenzklassen von Paaren von Matrizen ein, die einem linearen Fluss in $J(S) \backslash \Theta$ entsprechen. Jede Richtung $\beta \in H^{1}(S, \mathcal{O})$ definiert hierbei eine andere LaxPaar-Bedingung. Schließlich zeigen wir in Abschnitt 2.5, dass lineare Flüsse in der Jakobischen durch Punkte des Thetadivisors diesen sofort verlassen.

Kapitel 3 ist ein Hauptteil dieser Arbeit und weitgehend unabhängig von den beiden vorhergehenden Kapiteln. Wir geben in Abschnitt 3.1 eine kurze Einführung in die Painlevé-Analysis. Diese führen wir in den folgenden Abschnitten für die in Abschnitt 2.4 bewiesenen Lax-Paar-Gleichungen $\dot{C}=$ $[C, D]$ durch, wobei gilt $C(z)=A(z)+\zeta B(z)$. In Abschnitt 3.2 zeigen wir, dass für jede Richtung $\beta$ im zugehörigen Fluss $(A(z), B(z))$ die Matrix $B$ konstant ist, falls $A+\zeta B$ die Lax-Paar-Bedingung für $\beta$ erfüllt. In Abschnitt 3.3 wählen wir eine feste Richtung $\beta$ und berechnen die indizierende Menge zur zugehörigen Lax-Paar-Gleichung. In Abschnitt 3.4 bestimmen wir die sogenannte Kowalevski-Matrix, mit deren Hilfe weitere Koeffizienten formaler Laurentlösungen von $\dot{C}=[C, D]$ bestimmt werden können. Indem wir dies für ein ausgewähltes Element aus der indizierenden Menge in Abschnitt 3.5 durchführen, können wir zeigen, dass es sich bei der so berechneten Familie von formalen Laurentlösungen um eine Hauptbalance handelt. Durch Fixierung der Spektralkurve $S$ stellt sie das asymptotische Verhalten von Äquivalenzklassen von Paaren von Matrizen im allgemeinen Punkt des Thetadivisors in $J(S)$ entlang $\beta$ dar.

Zwei weitere Hauptteile dieser Arbeit sind Kapitel 4 und 5. Bevor wir diese im Detail beschreiben, wollen wir sie kurz motivieren: Mithilfe der Ergebnisse aus Kapitel 3 können wir in Kapitel 6 bereits jede konjugationsinvariante Funktion $f$ auf Paaren von Matrizen bis auf einen konstanten Term mittels 
Thetafunktionen als meromorphe Funktion $\widehat{f}: J(S) \rightarrow \mathbb{C}$ ausdrücken. Um diesen konstanten Term berechnen zu können, untersuchen wir das Verhalten von $f$ bzw. $\widehat{f}$ im konkreten Punkt $\mathcal{O}(m)$ des Thetadivisors. Zu einem linearen Fluss $L^{z}(m)$ durch $\mathcal{O}(m)$ definiert durch die Richtung $\beta \in H^{1}(S, \mathcal{O})$ bestimmen wir das asymptotische Verhalten der Urbilder in $M_{S}$. Hierzu müssen wir explizit auf die Identifizierung zwischen $M_{S}$ und $J(S) \backslash \Theta$ eingehen.

In Kapitel 4 beschäftigen wir uns mit holomorphen Schnitten des Vektorbündels $\mathcal{O}(m)$, die sich für $|z|$ hinreichend klein zu einer Familie von holomorphen Schnitten der Vektorbündel $L^{z}(m)$ fortsetzen lassen. Hier benötigen wir die konkreten Beschreibungen von $H^{1}(S, \mathcal{O}), H^{0}(S, \mathcal{O}(n)), n \in \mathbb{N}$, und $H^{1}(S, \mathcal{O}(m))$ aus Kapitel 2. Wir definieren in Abschnitt 4.1 ein Vektorbündel $V$, für das für allgemeines $z \neq 0$ gilt $V_{z}=H^{0}\left(S, L^{z}(m)\right)$. Wir interessieren uns folglich für $V_{0} \subset H^{0}(S, \mathcal{O}(m))$. In den Abschnitten 4.2 und 4.3 definieren wir Fortsetzungen von holomorphen Schnitten aus $H^{0}(S, \mathcal{O}(m))$ und drücken Fortsetzungen endlicher Ordnung $l$ als Schnitte eines Vektorbündels $F^{l}(m)$ über $S$ aus. Hierbei sind einige spezielle Fortsetzungen durch Schnitte eines Vektorbündels $E^{l}(n)$ über $\mathbb{P}^{1}$ mit passendem $n \in \mathbb{N}$ definiert, darum geht es in den Abschnitten 4.4 bis 4.8. In Abschnitt 4.9 stellen wir eine Beziehung zwischen den zurückgezogenen Bündeln $E^{l}(n)$ über $S$ und dem Bündel $F^{l}(m)$ her. Schließlich geben wir in Abschnitt 4.10 eine Basis von $H^{0}\left(S, F^{l}(m)\right)$ für $l<m$ an, mithilfe der wir in Abschnitt 4.11 eine genaue Beschreibung von $V_{0}$ erhalten. In Abschnitt 4.12 führen wir einen Algorithmus ein, mit dem wir zu jedem Schnitt aus $V_{0}$ Fortsetzungen zu Schnitten von $L^{z}(m)$ beliebiger Ordnung konstruieren können.

In Kapitel 5 definieren wir ein Vektorbündel $K$ über $\mathbb{C}$ vom Rang $k$, dessen Faser für allgemeines $z \neq 0$ gleich dem Kern der Multiplikationsabbildung

$$
\operatorname{mult}_{z}: H^{0}(S, \mathcal{O}(1)) \otimes H^{0}\left(S, L^{z}(m)\right) \rightarrow H^{0}\left(S, L^{z}(m+1)\right)
$$

ist. In Abschnitt 5.2 bestimmen wir $K_{0}$, d.h. die Schnitte aus dem Raum $H^{0}(S, \mathcal{O}(1)) \otimes V_{0} \subset H^{0}(S, \mathcal{O}(1)) \otimes H^{0}(S, \mathcal{O}(m))$, die sich für hinreichend kleines $|z|>0 \mathrm{zu}$ Elementen aus $K_{z}$ fortsetzen lassen. Durch solche konkreten Fortsetzungen, die in Abschnitt 5.3 mithilfe der Berechnungen aus Kapitel 4 ermittelt werden, können wir in Abschnitt 5.4 die Polordnungen und Residuen der zugehörigen Paare von Matrizen $A(z), B(z)$ bestimmen.

In Kapitel 6 geben wir schließlich als ein zentrales Ergebnis einen Algorithmus zur Beschreibung von konjugationsinvarianten Funktionen auf Paaren von Matrizen mittels Thetafunktionen an. Mithilfe der in Kapitel 3 berechneten Hauptbalance können wir Laurententwicklungen von konjugationsinvarianten Funktionen $f$ auf Paaren von Matrizen in allgemeinen Punkten des Thetadivisors in Richtung $\beta$ bestimmen. Dadurch stellen wir $f$ bis auf einen konstanten 
Term mittels Thetafunktionen dar. Zur Berechnung dieses konstanten Terms verwenden wir die Resultate aus den Kapiteln 4 und 5. Beispielhaft stellen wir die Funktion $\operatorname{tr}\left([A, B]^{2}\right)$ mittels Thetafunktionen dar.

Ein Ausblick schließt die Arbeit ab.

\section{Danksagung}

An dieser Stelle möchte ich mich bei Herrn Prof. Dr. Pidstrygach für die interessante Themenstellung, die hilfreichen Diskussionen und die gute Betreuung bedanken. Weiterhin danke ich dem Graduiertenkolleg „Gruppen und Geometrie" für die finanzielle Unterstützung. Dank geht auch an meine Kollegen Michael Miesener und Sven Porst für interessante Diskussionen und Anmerkungen zu dieser Arbeit. Andreas-Stephan Elsenhans und Michael Schachtebeck haben mühevoll nach Rechtschreib- und Kommafehler gesucht und mich in Layoutfragen unterstützt, vielen Dank auch hierfür. 



\section{Kapitel 1}

\section{Allgemeines}

In diesem Kapitel fassen wir bekannte und für unsere Berechnungen nützliche Aspekte von algebraischen Kurven und rationalen Regelflächen zusammen und legen Notationen fest. Weitergehende Details findet man z.B. in [GH78], [H77] und [F98].

In Abschnitt 1.3 wiederholen wir Eigenschaften von Thetafunktionen auf Jakobischen, bevor wir allgemein meromorphe Funktionen auf Jakobischen mit Polstellen höchstens in einem fest gewählten Riemannschen Thetadivisor beschreiben. Hier richten wir uns nach [GH78] und [M83].

In Abschnitt 1.4 wiederholen wir den Satz von Beauville, der die Isomorphie zwischen Äquivalenzklassen von Paaren von Matrizen mit fester Spektralkurve und der zugehörigen Jakobischen ohne dem Thetadivisor beweist. Details findet man z.B. in [Hi83] oder in [E03].

In Abschnitt 1.5 geben wir einen festen Punkt im Thetadivisor der Jakobischen einer Spektralkurve an.

Wir setzen grundlegende Begriffe der Algebraischen Geometrie, wie man sie z.B. in [GH78] Kapitel 0 und 1 findet, voraus.

\subsection{Rationale Regelflächen}

Sei $R$ der Totalraum des tautologischen Geradenbündels $\mathcal{O}_{\mathbb{P}^{1}}(1)$ über $\mathbb{P}^{1}$ und $R \stackrel{\pi_{R}}{\rightarrow} \mathbb{P}^{1}$ die kanonische Projektion.

Die Kompaktifizierung $\widehat{R}=\mathbb{P}\left(\mathcal{O}_{\mathbb{P}^{1}}(1) \oplus \mathcal{O}\right)$ von $R$ ist eine rationale Regelfläche. Hierin ist die Faser $R_{\left(\zeta_{0}: \zeta_{1}\right)} \subset \widehat{R}$ von $R$ für $\left(\zeta_{0}: \zeta_{1}\right) \in \mathbb{P}^{1}$ gegeben durch

$$
\left\{(v: 1) \mid v \in\left(\mathcal{O}_{\mathbb{P}^{1}}(1)\right)_{\left(\zeta_{0}: \zeta_{1}\right)}, 1 \in\left(\mathcal{O}_{\mathbb{P}^{1}}\right)_{\left(\zeta_{0}: \zeta_{1}\right)}\right\} .
$$




\section{Divisoren in $\widehat{R}$}

Sei $\pi_{\widehat{R}}: \widehat{R} \rightarrow \mathbb{P}^{1}$ die kanonische Projektion und $\mathcal{O}_{\widehat{R}}(1)$ das relative tautologische Bündel über $\widehat{R}$. Sei $\mathcal{O}_{\widehat{R}}(1,0):=\pi_{\widehat{R}}^{*} \mathcal{O}_{\mathbb{P}^{1}}(1), \mathcal{O}_{\widehat{R}}(0,1):=\mathcal{O}_{\widehat{R}}(1)$ und damit $\mathcal{O}_{\widehat{R}}(1,1):=\pi_{\widehat{R}}^{*} \mathcal{O}_{\mathbb{P}^{1}}(1) \otimes \mathcal{O}_{\widehat{R}}(1)$.

Wir beschreiben im Folgenden einige für spätere Berechnungen wichtige Divisoren in $\widehat{R}$ genauer. Dabei nutzen wir die allgemeine Tatsache, dass jeder Bündelhomomorphismus

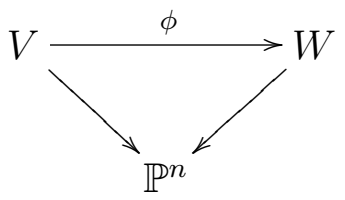

eindeutig einem Schnitt $s_{\phi} \in H^{0}\left(\mathbb{P}(V), \pi_{\mathbb{P}(V)}^{*} W \otimes \mathcal{O}_{\mathbb{P}(V)}(1)\right)$ entspricht, wobei für jedes $p \in \mathbb{P}^{n}$ gilt: $\left(s_{\phi}\right)_{0} \cap\left(\left.\mathbb{P}(V)\right|_{p}\right)=\mathbb{P}\left(\operatorname{ker}\left(\phi_{p}\right)\right)$. Hierbei ist die Abbildung $\pi_{\mathbb{P}(V)}: \mathbb{P}(V) \rightarrow \mathbb{P}^{n}$ die kanonische Projektion und $\mathcal{O}_{\mathbb{P}(V)}(1)$ das relative tautologische Geradenbündel über $\mathbb{P}(V)$.

1. Es bezeichne $E_{0}$ den Nullschnitt in $\widehat{R}$. Dieser Divisor ist durch den mittels des Bündelhomomorphismus

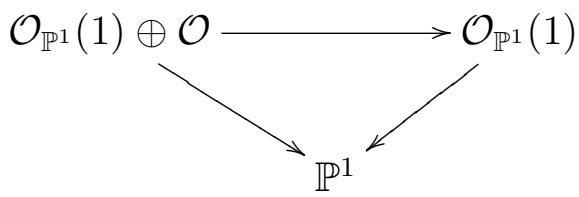

definierten Schnitt $s \in H^{0}\left(\widehat{R}, \mathcal{O}_{\widehat{R}}(1,1)\right)$ definiert. Es gilt $E_{0}=(s)_{0}$ und $(s)_{0}=\left\{(0: w) \mid 0 \in\left(\mathcal{O}_{\mathbb{P}^{1}}(1)\right)_{\left(\zeta_{0}: \zeta_{1}\right)}, 0 \neq w \in\left(\mathcal{O}_{\mathbb{P}^{1}}\right)_{\left(\zeta_{0}: \zeta_{1}\right)},\left(\zeta_{0}: \zeta_{1}\right) \in \mathbb{P}^{1}\right\}$.

Das Geradenbündel $\left[E_{0}\right]$ ist gleich $\mathcal{O}_{\widehat{R}}(1,1)$.

2. Ist $\sigma$ ein holomorpher Schnitt von $\mathcal{O}_{\mathbb{P}^{1}}(1)$, so definieren wir einen Divisor $E_{\sigma} \subset \widehat{R}$ durch $E_{\sigma}=\left\{(\sigma: 1) \mid \sigma\left(\zeta_{0}: \zeta_{1}\right) \in\left(\mathcal{O}_{\mathbb{P}^{1}}(1)\right)_{\left(\zeta_{0}: \zeta_{1}\right)}, 1 \in\left(\mathcal{O}_{\mathbb{P}^{1}}\right)_{\left(\zeta_{0}: \zeta_{1}\right)}\right\}$ mit jeweils $\left(\zeta_{0}: \zeta_{1}\right) \in \mathbb{P}^{1}$. Die Divisoren $E_{0}$ und $E_{\sigma}$ sind äquivalent, und für die zugehörigen Geradenbündel gilt $\left[E_{\sigma}\right]=\left[E_{0}\right]=\mathcal{O}_{\widehat{R}}(1,1)$.

3. Bezeichne $C$ eine Faser in $\widehat{R} \stackrel{\pi_{\widehat{R}}}{\longrightarrow} \mathbb{P}^{1}$. $C$ ist dann gleich $(t)_{0}$ für einen Schnitt $t \in H^{0}\left(\widehat{R}, \pi_{\widehat{R}}^{*} \mathcal{O}_{\mathbb{P}^{1}}(1)\right)$. Das Geradenbündel $[C]$ ist gleich $\mathcal{O}_{\widehat{R}}(1,0)$.

Die Divisoren $C, E_{0}$ und $E_{\sigma}$ in $\widehat{R}$ skizzieren wir in Abbildung 1.1. 
4. Sei $E_{\infty}$ die Nullstellenmenge des durch den Bündelhomomorphismus

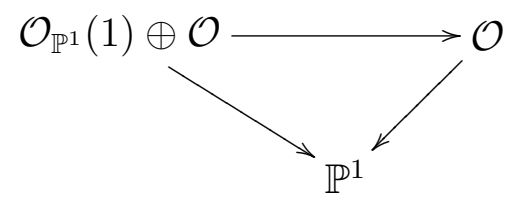

definierten Schnittes $q \in H^{0}\left(\widehat{R}, \mathcal{O}_{\widehat{R}}(0,1)\right)$. Dann ist das Geradenbündel $\left[E_{\infty}\right]$ gleich $\mathcal{O}_{\widehat{R}}(0,1)$. Für $\sigma \in H^{0}\left(\mathbb{P}^{1}, \mathcal{O}_{\mathbb{P}^{1}}(1)\right)$ definiert der Schnitt $(\sigma, 0)$ von $\mathcal{O}_{\mathbb{P}^{1}}(1) \oplus \mathcal{O}$ außerhalb der Nullstellen von $\sigma$ eine Kurve in $\widehat{R}$, der Abschluß dieser Kurve ist dann $E_{\infty}$.

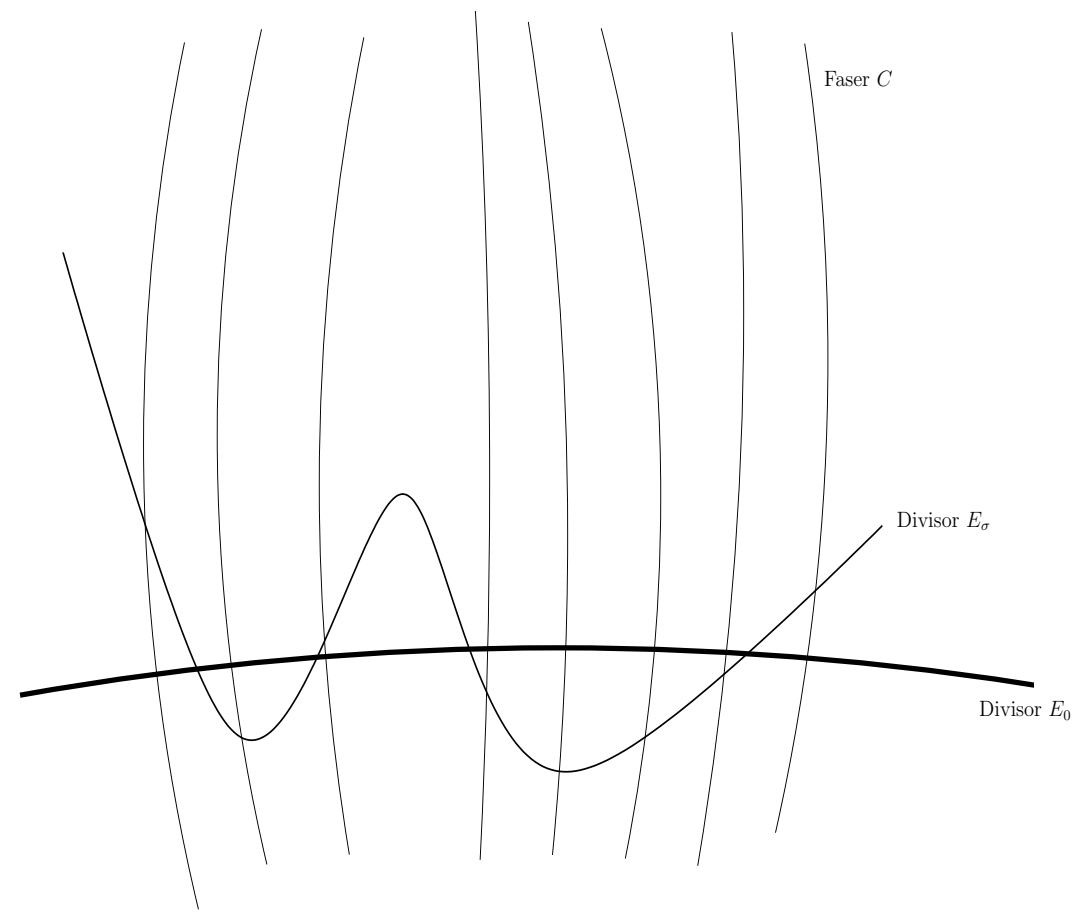

Abbildung 1.1: Beispiel für eine allgemeine Regelfläche $\mathbb{P}(\mathcal{O}(d) \oplus \mathcal{O})$ mit $d \in \mathbb{N}$. In diesem Fall schneiden sich $E_{0}$ und $E_{\sigma}$ in genau $d$ Punkten.

Für die Schnittzahlen gilt:

$$
\begin{aligned}
E_{0} \cdot E_{0} & =1 \\
E_{0} \cdot E_{\infty} & =0 \\
E_{0} \cdot C & =E_{\sigma} \cdot C=E_{\infty} \cdot C=1 \\
C \cdot C & =0 .
\end{aligned}
$$


Da $\widehat{R}$ eine rationale Regelfläche ist, wird $\operatorname{Pic}(\widehat{R})$ durch die Geradenbündel $\mathcal{O}_{\widehat{R}}(1,1)=\left[E_{0}\right]$ und $\mathcal{O}_{\widehat{R}}(1,0)=[C]$ erzeugt. Genauso erzeugen die Geradenbündel $\mathcal{O}_{\widehat{R}}(1,0)=[C]$ und $\mathcal{O}_{\widehat{R}}(0,1)=\left[E_{\infty}\right]$ die Picardgruppe $\operatorname{Pic}(\widehat{R})$ (siehe [GH78] S. 518). Insbesondere gibt es ganze Zahlen $a$ und $b$ mit $E_{\infty}=$ $a E_{0}+b C$. Mit den Schnittzahlen (1.1)-(1.4) folgt $a=1$ und $b=-1$, d.h.

$$
E_{\infty}=E_{0}-C
$$

und damit

$$
E_{\infty} \cdot E_{\infty}=-1
$$

Außerdem folgt mit der Adjunktionsformel ([GH78] S. 519) für den kanonischen Divisor $K_{\widehat{R}}$ :

$$
\begin{aligned}
& 0=g(C)=\frac{C \cdot C+C \cdot K_{\widehat{R}}}{2}+1 \Rightarrow C \cdot K_{\widehat{R}}=-2 \\
& 0=g\left(E_{0}\right)=\frac{E_{0} \cdot E_{0}+E_{0} \cdot K_{\widehat{R}}}{2}+1 \Rightarrow E_{0} \cdot K_{\widehat{R}}=-2-1
\end{aligned}
$$

und damit

$$
K_{\widehat{R}}=-2 E_{0}-C
$$

\section{Verschwindungssatz von Kodaira}

Zur Berechnung von Kohomologieklassen ist der Verschwindungssatz von Kodaira für ample Geradenbündel auf algebraischen Flächen nützlich:

Satz 1.1. ([F98] S. 22) Sei D ein ampler Divisor auf einer glatten algebraischen Fläche $X$. Dann gilt $H^{i}(X,[-D])=0$ für $i=0,1$. Außerdem gilt dual $H^{i}\left(X,\left[K_{X}+D\right]\right)=0$ für $i=1,2$.

Für die amplen Geradenbündel auf der rationalen Regelfläche $\widehat{R}$ gibt es eine einfache Beschreibung:

Satz 1.2. ([H'ry] S. 365) (Kriterium von Nakai-Moishezon) Ein Divisor D auf der Fläche $X$ ist genau dann ampel, wenn $D^{2}>0$ und $D \cdot F>0$ für alle irreduziblen Kurven $F$ in $X$ gilt.

Für die oben definierten Divisoren $E_{\infty}$ und $C$ gilt:

Lemma 1.3. ([F98] S. 116) Für ganze Zahlen $a, b \in \mathbb{Z}$ ist das Geradenbündel $a E_{\infty}+b C$ über der rationale Regelfläche $\widehat{R}$ genau dann ampel, wenn $b>a>0$ gilt. 


\subsection{Die Spektralkurve in $\widehat{R}$}

Wir betrachten die Menge aller Äquivalenzklassen von Paaren von Matrizen $\left(\mathrm{gl}_{k} \times \mathrm{gl}_{k}\right) / \mathrm{GL}_{k}$ mit festem $k \in \mathbb{N}$.

Ist $(A, B) \in\left(\mathrm{gl}_{k} \times \mathrm{gl}_{k}\right)$, so bezeichnet das Polynom $A+\zeta B$ einen Schnitt aus $H^{0}\left(\mathbb{P}^{1},\left(\mathbb{C}^{k} \otimes\left(\mathbb{C}^{k}\right)^{\vee}\right) \otimes \mathcal{O}_{\mathbb{P}^{1}}(1)\right)$. Sei $\eta \in H^{0}\left(R, \pi_{R}^{*} \mathcal{O}_{\mathbb{P}^{1}}(1)\right)$ der tautologische Schnitt. Bezeichnet $A+\zeta B$ ebenfalls den Schnitt $\pi_{R}^{*}(A+\zeta B)$ in dem Raum $H^{0}\left(R,\left(\mathbb{C}^{k} \otimes\left(\mathbb{C}^{k}\right)^{\vee}\right) \otimes \pi_{R}^{*} \mathcal{O}_{\mathbb{P}^{1}}(1)\right)$, so ist $\psi:=\operatorname{det}\left((A+\zeta B)+\eta \mathbb{1}_{k}\right)$ von der Form

$$
\psi=\eta^{k}+a_{1}(\zeta) \eta^{k-1}+\ldots+a_{k}(\zeta) \in H^{0}\left(R, \pi_{R}^{*} \mathcal{O}_{\mathbb{P}^{1}}(k)\right)
$$

mit $a_{i}(\zeta) \in \pi_{R}^{*} H^{0}\left(\mathbb{P}^{1}, \mathcal{O}(i)\right)$. Insbesondere ist $a_{1}(\zeta)=\operatorname{tr}(A+\zeta B)=\operatorname{tr} A+\zeta \operatorname{tr} B$ und $a_{k}(\zeta)=\operatorname{det}(A+\zeta B)$.

$\eta \in H^{0}\left(R, \pi_{R}^{*} \mathcal{O}_{\mathbb{P}^{1}}(1)\right)$ besitzt eine kanonische Fortsetzung zu einem Schnitt $\widehat{\eta} \in H^{0}\left(\widehat{R},\left[E_{0}\right]\right)$. Mit $\widehat{\xi} \in H^{0}\left(\widehat{R},\left[E_{\infty}\right]\right)$ gibt es dann eine Fortsetzung von $\psi \in H^{0}\left(R, \pi_{R}^{*} \mathcal{O}_{\mathbb{P}^{1}}(k)\right) \mathrm{zu}$

$$
\widehat{\psi}=\widehat{\eta}^{k}+a_{1} \widehat{\eta}^{k-1} \widehat{\xi}+a_{2} \widehat{\eta}^{k-2} \widehat{\xi}^{2}+\ldots+a_{k} \widehat{\xi}^{k} \in H^{0}\left(\widehat{R},\left[k E_{0}\right]\right)
$$

Geometrisch ist die Konstruktion die folgende: Wir betrachten den Bündelhomomorphismus

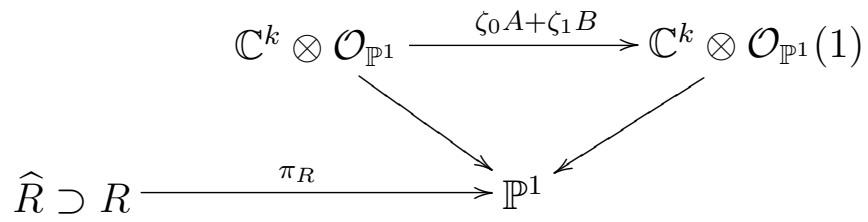

Sei $h:=\pi_{R}^{*}\left(\zeta_{0} A+\zeta_{1} B\right)+\eta \mathbb{1}_{k}$, also

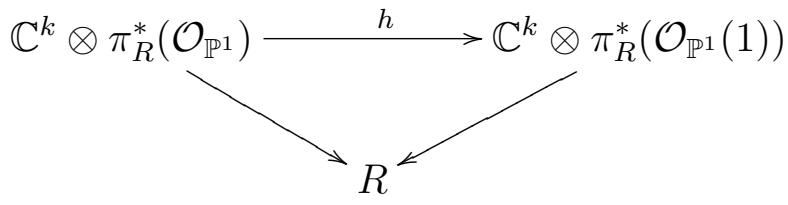

und dementsprechend $\widehat{h}:=\widehat{\xi} \pi_{\widehat{R}}^{*}\left(\zeta_{0} A+\zeta_{1} B\right)+\widehat{\eta} \mathbb{1}_{k}$ :

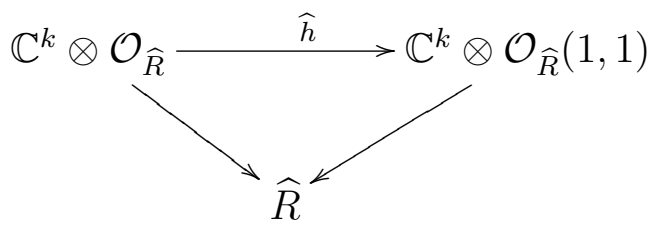


Dann ist $\widehat{\psi}=\operatorname{det}\left(\widehat{\xi} \pi^{*}\left(\zeta_{0} A+\zeta_{1} B\right)+\widehat{\eta} \mathbb{1}\right) \in H^{0}\left(\widehat{R},\left[k E_{0}\right]\right)=H^{0}\left(\widehat{R}, \mathcal{O}_{\widehat{R}}(k, k)\right)$ gleich dem Schnitt $\sigma_{\Lambda^{k} \widehat{h}}$ zum Bündelhomomorphismus

$$
\Lambda^{k} \widehat{h}: \Lambda^{k} \mathbb{C}^{k} \otimes \mathcal{O}_{\widehat{R}}=\mathcal{O}_{\widehat{R}} \rightarrow \Lambda^{k} \mathbb{C}^{k} \otimes \mathcal{O}_{\widehat{R}}(k, k)=\mathcal{O}_{\widehat{R}}(k, k) .
$$

Definition und Satz 1.4. Die Spektralkurve $S$ zur Äquivalenzklasse des Paares $(A, B) \in g l_{k} \times g l_{k}$ sei der Divisor $S:=S_{(A, B)}:=(\widehat{\psi})_{0} \subset \widehat{R}$ in der rationalen Regelfläche $\widehat{R}$. S ist eine algebraische Kurve vom Grad $k$, die vollständig in $R$ enthalten ist. Sie hängt nur von der Äquivalenzklasse von $(A, B) a b$.

Bemerkung 1.5. Die Schnitte des Geradenbündels $\pi_{R}^{*} \mathcal{O}_{\mathbb{P}^{1}}(1)$ definieren eine Einbettung $\iota: R \rightarrow \mathbb{P}^{2}$. Hierbei liegt der Punkt $p:=(0: 0: 1) \in \mathbb{P}^{2}$ nicht im Bild $\iota(R)$. Die rationale Regelfläche $\widehat{R}$ entspricht der Aufblasung von $\mathbb{P}^{2}$ im Punkt p. Das Bild von $S$ unter der Abbildung $\iota$ ist gleich der Spektralkurve $\Sigma=\left\{\left(\nu_{1}: \nu_{2}: \mu\right) \in \mathbb{P}^{2} \mid \operatorname{det}\left(\mu \mathbb{1}+\nu_{1} A+\nu_{2} B\right)=0\right\} \subset \mathbb{P}^{2}$. Wir betrachten in dieser Arbeit stets die in $R$ bzw. der rationalen Regelfläche $\widehat{R}$ eingebettete Kurve $S$, da dies insbesondere die konkreten Berechnungen von Erweiterungen von Schnitten von $L^{z}(m)$ in Kapitel 4 erlaubt.

Das Geschlecht von $S$ läßt sich für nichtsinguläre $S$ mit der Adjunktionsformel ermitteln:

$$
\begin{aligned}
g(S) & =\frac{S \cdot S+S \cdot K_{\widehat{R}}}{2}+1 \\
& =\frac{\left(k E_{0}\right) \cdot\left(k E_{0}\right)+\left(k E_{0}\right) \cdot\left(-2 E_{0}-C\right)}{2}+1 \\
& =\frac{1}{2}(k-1)(k-2) .
\end{aligned}
$$

\subsection{Thetafunktionen}

\section{Geradenbündel definiert durch Multiplikatoren}

Sei $T^{r}:=\mathbb{C}^{r} / \Lambda$ ein komplexer $r$-dimensionaler Torus mit Gitter $\Lambda$ und Projektion $\pi_{T^{r}}: \mathbb{C}^{r} \rightarrow T^{r}$. Sei $\mathcal{L}$ ein beliebiges Geradenbündel über $T^{r}$. Dann ist das zurückgezogene Geradenbündel $\pi_{T^{r}}^{*} \mathcal{L}$ über $\mathbb{C}^{r}$ trivial. Sei $\phi: \pi_{T^{r}}^{*} \mathcal{L} \rightarrow \mathbb{C}^{r} \times \mathbb{C}$ eine globale Trivialsisierung. Da für $z \in \mathbb{C}^{r}$ und $\lambda \in \Lambda$ die Fasern $\left.\pi_{T^{r}}^{*} \mathcal{L}\right|_{z}$ und $\left.\pi_{T^{r}}^{*} \mathcal{L}\right|_{z+\lambda}$ übereinstimmen, definiert

$$
z \times \mathbb{C} \stackrel{\left.\phi_{z}:=\left.\phi\right|_{\left(\pi_{T}^{*}\right.} ^{*} \mathcal{L}\right) z}{\longleftarrow}\left(\pi_{T^{r}}^{*} \mathcal{L}\right)_{z}=\left(\pi_{T^{r}}^{*} \mathcal{L}\right)_{z+\lambda} \stackrel{\phi_{z+\lambda}}{\longrightarrow}(z+\lambda) \times \mathbb{C}
$$


einen Automorphismus $e_{\lambda}(z):=\phi_{z+\lambda} \circ \phi_{z}^{-1}: \mathbb{C} \rightarrow \mathbb{C}$ mit $w \mapsto e_{\lambda}(z) \cdot w$ und damit eine Menge von Multiplikatoren $\left\{e_{\lambda} \in \mathcal{O}^{*}\left(\mathbb{C}^{r}\right) \mid \lambda \in \Lambda\right\}$, die

$$
e_{\lambda^{\prime}}(z+\lambda) e_{\lambda}(z)=e_{\lambda}\left(z+\lambda^{\prime}\right) e_{\lambda^{\prime}}(z)=e_{\lambda+\lambda^{\prime}}(z)
$$

erfüllen. Umgekehrt definieren Multiplikatoren $\left\{e_{\lambda}\right\}_{\lambda \in \Lambda}$, für die (1.6) gilt, eindeutig ein Geradenbündel $\mathcal{L} \rightarrow T^{r}$ mit diesen Multiplikatoren: $\mathcal{L}$ sei der Quotientenraum von $\mathbb{C}^{r} \times \mathbb{C}$ unter der Identifizierung $(z, w) \sim\left(z+\lambda, e_{\lambda}(z) \cdot w\right)$. Holomorphe Schnitte des Geradenbündels $\mathcal{L}$ sind dann definiert durch holomorphe Funktionen $\vartheta: \mathbb{C}^{r} \rightarrow \mathbb{C}$, die $\vartheta(z+\lambda)=e_{\lambda}(z) \vartheta(z)$ für alle $z \in \mathbb{C}^{r}$ und $\lambda \in \Lambda$ erfüllen (Details siehe z.B. [GH78] S. 307 ff.).

\section{Riemannsche Thetafunktionen}

Jede Jakobische $J(S)$ einer kompakten Riemannschen Fläche $S$ besitzt eine Periodenmatrix $\Pi$ der Gestalt $\Pi=\left(\mathbb{1}_{g}, Z\right)$ mit $Z^{t}=Z$ und $\operatorname{Im}(Z)$ positiv definit. Damit ist jede Jakobische eine Abelsche Varietät, die eine Hauptpolarisierung, d.h. ein positives Geradenbündel $\mathcal{L}$ mit $c_{1}(\mathcal{L})=[\omega]$ und $\omega$ von der Form $\sum_{l=1}^{g} d x_{l} \wedge d x_{g+l}$, besitzt (Details zu Abelschen Varietäten und Polarisierungen siehe zum Beispiel [GH78] S. 300 ff.).

Angenommen ein komplexer Torus $T^{r}=\mathbb{C}^{r} / \Lambda$ ist eine Abelsche Varietät mit Polarisierung $\omega=\sum_{l=1}^{r} \delta_{l} d x_{l} \wedge d x_{r+l}, \delta_{l} \mid \delta_{l+1}$ mit einer geeigneten reellen Basis $x_{1}, \ldots x_{2 r}$ von $\mathbb{C}^{r} \cong \mathbb{R}^{2 r}$. Sei $\lambda_{1}, \ldots, \lambda_{2 r}$ die ganzzahlige Basis von $\Lambda$ mit $d x_{l}\left(\lambda_{j}\right)=\delta_{l j}$, und $z_{1}, \ldots, z_{r}$ lineare komplexe Koordinaten von $\mathbb{C}^{r}$ definiert durch $d z_{j}\left(\lambda_{l}\right)=\delta_{j} \delta_{l j}$. Dann gilt:

Satz 1.6. [GH78] Das Geradenbündel $\mathcal{L}$, definiert durch die Multiplikatoren $e_{\lambda_{j}}(z)=1$ und $e_{\lambda_{r+j}}(z)=e^{-2 \pi i z_{j}}$ für $j=1, \ldots, r$ hat $c_{1}(\mathcal{L})=[\omega]$. Alle Geradenbündel mit fester Chernklasse sind bis auf Translation eindeutig bestimmt.

Aufgrund der Definition des Geradenbündels $\mathcal{L}$ durch die Multiplikatoren $e_{\lambda_{j}}(z)=1$ und $e_{\lambda_{r+j}}(z)=e^{-2 \pi i z_{j}}$ für $j=1, \ldots, r$ sind Schnitte des Geradenbündels $\mathcal{L}$ eindeutig definiert durch Thetafunktionen, d.h. holomorphe Funktionen $\vartheta: \mathbb{C}^{r} \rightarrow \mathbb{C}$, die $\vartheta\left(z+\lambda_{j}\right)=\vartheta(z)$ und $\vartheta\left(z+\lambda_{j+r}\right)=e^{-2 \pi i z_{j}} \vartheta(z)$ für $j=1, \ldots, r$ erfüllen. Es gilt

Satz 1.7. [GH78] Sei $\mathcal{L} \rightarrow T^{r}$ ein positives Geradenbündel mit Elementarteiler $\delta_{1}, \ldots, \delta_{r}$ der Polarisierung $c_{1}(\mathcal{L})$ von $T^{r}$. Dann gilt: $\operatorname{dim} H^{0}\left(T^{r}, \mathcal{O}(\mathcal{L})\right)=$ $\prod_{l=1}^{r} \delta_{l}$

Ist insbesondere $c_{1}(\mathcal{L})$, wie im Fall für die Jakobische $T^{g}=J(S)$ einer algebraischen Kurve $S$, eine Hauptpolarisierung von $T^{g}$ mit Periodenmatrix $(1, Z)$, 
so wird der eindimensionale Raum $H^{0}\left(T^{g}, \mathcal{O}(\mathcal{L})\right)$ erzeugt durch den Schnitt $\tilde{\vartheta}$, der definiert ist durch die holomorphe Funktion

$$
\vartheta: \mathbb{C}^{g} \rightarrow \mathbb{C}, \quad \vartheta(z):=\vartheta(z, Z)=\sum_{l \in \mathbb{Z}^{g}} e^{\pi i<l, Z l>} \cdot e^{2 \pi i<l, z>}
$$

Dies ist die sogenannte Riemannsche Thetafunktion. Multiplikatoren von $\mathcal{L}$ sind hier $e_{\lambda_{j}}=1$ und $e_{\lambda_{j+g}}(z)=e^{-2 \pi i\left(z_{j}+\frac{z_{j j}}{2}\right)}$. Der zugehörige Riemannsche Thetadivisor $\Theta=(\tilde{\vartheta})_{0}=\left((\vartheta)_{0}\right.$ modulo $\left.\Lambda\right)$ ist bis auf Translation eindeutig durch die Hauptpolarisierung definiert (Details zu diesem Abschnitt siehe [GH78] S. 310 ff.).

\section{Thetadivisoren auf der Jakobischen}

Sei $\Theta$ der Riemannsche Thetadivisor in $J(S)$. Die Jakobische $J(S)$ ist via der Abel-Jakobi-Abbildung $u_{d}: \operatorname{Pic}^{d}(S) \rightarrow J(S)$ isomorph zu $J^{d}(S):=\operatorname{Pic}^{d}(S)$, wobei der Isomorphismus für $d \neq 0$ von der Wahl eines Basispunktes in $S$ abhängt. Wir bezeichnen im Folgenden mit $\Theta \subset J^{d}(S)$ das Urbild des Thetadivisors $\Theta \subset J(S)$ unter der Abel-Jacobi-Abbildung $u_{d}$. Bezeichnen wir mit $W_{g-1}(S) \subset J^{g-1}(S)$ den Divisor in $J^{g-1}(S)$ aller Geradenbündel vom Grad $g-1$ über $S$, die nichttriviale globale holomorphe Schnitte besitzen, so gilt der Satz von Riemann:

Satz 1.8. [ACGH85] Es gibt einen Punkt $\kappa \in J(S)$, so dass $W_{g-1}(S)=\Theta-\kappa$ gilt.

Hierbei hängt die Riemannsche Konstante $\kappa$ ebenfalls von einem Basispunkt $p \in S$ bzw. einem ausgewählten Punkt in $J^{g-1}(S)$ ab.

Außerdem gilt

Satz 1.9. (Riemannscher Singularitätensatz[ACGH85]) Ist S eine glatte Kurve vom Geschlecht $g$, so gilt mult $_{L+\kappa}(\Theta)=h^{0}(S, \mathcal{O}(L))$ für jedes Geradenbündel $L \in W_{g-1} \subset J^{g-1}(S)$.

Wir bezeichnen zur Vereinfachung im Folgenden mit $\Theta$ ebenfalls den Divisor $W_{g-1} \subset J^{g-1}(S)$. Aus dem Kontext ist in diesen Fällen ersichtlich, ob der Riemannsche Thetadivisor $\Theta$ oder der um die Riemannsche Konstante $-\kappa$ verschobene Riemannsche Thetadivisor, d.h. $W_{g-1}$, gemeint ist. 


\section{Meromorphe Funktionen auf $J(S)$}

Wir interessieren uns für meromorphe Funktionen auf der Jakobischen $J(S)$ mit Periodenmatrix $(1, Z)$ mit Polstellen im Thetadivisor $\Theta \subset J(S)$, d.h. holomorphe Schnitte von $H^{0}\left(J(S), \mathcal{O}_{J(S)}(n \Theta)\right)$ für ein $n \in \mathbb{N}$. Das Geradenbündel $[n \Theta]$ ist durch die Multiplikatoren $e_{\lambda_{j}}=1$ und $e_{\lambda_{j+g}}=e^{-2 \pi n i\left(z_{j}+\frac{z_{j j}}{2}\right)}$ definiert. Der Raum der Schnitte $H^{0}(J(S), \mathcal{O}(n \Theta))$ ist damit isomorph zum Raum der Thetafunktionen $R_{n}^{Z}$ vom Gewicht $n$, d.h. holomorphen Funktionen $\vartheta: \mathbb{C}^{g} \rightarrow \mathbb{C}$, die $\vartheta\left(z+\lambda_{j}\right)=\vartheta(z)$ und $\vartheta\left(z+\lambda_{j+g}\right)=e^{-2 \pi n i\left(z_{j}+\frac{z_{j j}}{2}\right)} \vartheta(z)$ für $j=1, \ldots, g$ erfüllen. Für $R_{n}^{Z}$ kann man eine Basis durch sogenannte Thetafunktionen $\vartheta\left[\begin{array}{l}a \\ b\end{array}\right](z, Z)$ mit rationalen Charakteristiken $a$ und $b$ angeben (Details siehe [M83] Kapitel 2). Diese sind für $a, b \in \mathbb{Q}^{g}$ durch

$$
\vartheta\left[\begin{array}{l}
a \\
b
\end{array}\right](z, Z):=\sum_{l \in \mathbb{Z}^{g}} e^{\pi i<l+a, Z(l+a)>} \cdot e^{2 \pi i<l+a, z+b>}
$$

definiert. Es gilt

Satz 1.10. [M83] Für ein festes $n \in \mathbb{N}$ definieren für $a \in \mathbb{N}^{g}$ mit $0 \leq a_{i}<n$ die $n^{g}$ Funktionen

$$
h_{a}(z):=\vartheta\left[\begin{array}{l}
\frac{a}{n} \\
0
\end{array}\right](n z, n Z)
$$

eine Basis von $R_{n}^{Z}$

Bemerkung 1.11. Für $b \in \mathbb{N}^{g}$ mit $0 \leq b_{i}<n$ definieren die $n^{g}$ Funktionen

$$
\widetilde{h}_{b}(z):=\vartheta\left[\begin{array}{l}
0 \\
\frac{b}{n}
\end{array}\right]\left(z, \frac{1}{n} Z\right)
$$

ebenfalls eine Basis von $R_{n}^{Z}$. Ist $n=l^{2}$ für ein $l \in \mathbb{N}$, so definieren für $a, b \in \mathbb{N}^{g}$ mit $0 \leq a_{i}, b_{i}<l$ die Funktionen

$$
\widehat{h}_{a, b}(z):=\vartheta\left[\begin{array}{c}
\frac{a}{l} \\
\frac{b}{l}
\end{array}\right](l z, Z)
$$

eine dritte Basis von $R_{n}^{Z}$. Die verschiedenen Basen $h_{a}, \widetilde{h}_{b}$ und $\widehat{h}_{a, b}$ erfüllen $\widetilde{h}_{b}=\sum_{a} e^{2 \pi i n^{-1}<a, b>} h_{a}$ bzw. $\widehat{h}_{a, b}=\sum_{c \equiv a \bmod l} e^{2 \pi i l^{-1}<c, b>} h_{c}$.

Mit der Identifizierung

$$
H^{0}(J(S), \mathcal{O}(n \Theta)) \cong L(n \Theta)=\{f: J(S) \rightarrow \mathbb{C} \mid f \text { meromorph, }(f) \geq-n \Theta\}
$$

erhalten wir so eine Beschreibung aller meromorphen Funktionen $f$ auf $J(S)$ mit Polstellen im Thetadivisor: eine solche meromorphe Funktion $f$ ist gleich $\frac{g}{\vartheta^{n}}$ für ein $g \in R_{n}^{Z}, n \in \mathbb{N}$. 
Beispiel 1.12. Für $n=2$ ist für eine feste Riemannsche Thetafunktion $\vartheta: \mathbb{C}^{g} \rightarrow \mathbb{C}$ zum Gitter $\Lambda=\left(\mathbb{1}_{g} Z\right)$ die Funktion

$$
\frac{d^{2}}{d z_{a} d z_{b}} \log \vartheta=\frac{\vartheta \frac{d^{2} \vartheta}{d z_{a} d z_{b}}-\left(\frac{d \vartheta}{d z_{a}} \frac{d \vartheta}{d z_{b}}\right)}{\vartheta^{2}}
$$

mit $1 \leq a, b \leq g$ eine meromorphe Funktion auf $\mathbb{C}^{g} / \Lambda$ mit Polen im Thetadivisor. Hierbei ist der Zähler $\mathrm{z}(z)$ der Funktion $\frac{d^{2}}{d z_{a} d z_{b}} \log \vartheta$ eine quasiperiodische Funktion mit $\mathbf{z}\left(z+\lambda_{j}\right)=\mathbf{z}(z)$ und $\mathbf{z}\left(z+\lambda_{j+g}\right)=e^{2 \cdot\left(-2 \pi i\left(z_{j}+\frac{z_{j j}}{2}\right)\right.} \mathbf{z}(z)$ für $1 \leq j \leq g$, d.h. ein Element aus $R_{2}^{Z}$, und kann deshalb mittels Funktionen $h_{a}$ dargestellt werden.

Weitere Beispiele findet man in [M83] Kapitel 2.3.

\subsection{Der Satz von Beauville}

Sei $(A, B) \in\left(\mathrm{gl}_{k} \times \mathrm{gl}_{k}\right) / G L_{k}$ fest gewählt mit nichtsingulärer Spektralkurve $S:=S_{(A, B)}$. Diese ist eine Invariante zu Äquivalenzklassen von Paaren von Matrizen. Sie ist jedoch keine vollständige Invariante, d.h. zu $(A, B)$ gibt es nicht-äquivalente Paare $(C, D) \in \mathrm{gl}_{k} \times \mathrm{gl}_{k}$ mit der gleichen Spektralkurve $S$.

Sei $M_{S} \subset\left(\mathrm{gl}_{k} \times \mathrm{gl}_{k}\right) / G L_{k}$ der Raum aller Äquivalenzklassen von Paaren von Matrizen mit fester nichtsingulärer Spektralkurve $S$. Dann gilt

Satz 1.13. ([B90]) $M_{S}$ kann mit der Jakobischen ohne dem Thetadivisor, $J^{k+g-1}(S) \backslash \Theta$, identifiziert werden.

Ein ausführlicher Beweis wird in [E03] gegeben.

Die Identifizierung erfolgt darüber, dass jeder Äquivalenzklasse $(C, D)$ von Paaren von Matrizen ein Eigenvektorbündel $L_{C, D} \in J^{k+g-1} \backslash \Theta$ vom Grad $k+g-1$ zugeordnet wird. Eine Faser des Eigenvektorbündels über einem allgemeinen Punkt $(\eta, \zeta) \in S$ entspricht hierbei dem Dualraum des Eigenraumes von $C+\zeta D$.

Umgekehrt ist für jedes Geradenbündel $L \in J^{k+g-1}(S) \backslash \Theta$ der Raum $H^{0}(S, L)$ k-dimensional. Wir verwenden in dieser Arbeit die Konstruktion einer Äquivalenzklasse von Paaren von Matrizen zu $L$ durch

Satz 1.14. ([Hi83]) Sei mult : $H^{0}\left(S, \pi_{S}^{*} \mathcal{O}_{\mathbb{P}^{1}}(1)\right) \otimes H^{0}(S, L) \rightarrow H^{0}(S, L(1))$ die Multiplikationsabbildung. Dann ist der Homomorphismus $h: \operatorname{Ker}(\operatorname{mult}) \rightarrow$ $H^{0}(S, L)$, der $\eta \otimes s_{0}+1 \otimes s_{1}+\zeta \otimes s_{2}$ mit $s_{0}, s_{1}$ und $s_{2}$ aus $H^{0}(S, L)$ auf $s_{0}$ abbildet, ein Isomorphismus. 
Dadurch sind eindeutig Endomorphismen $\widetilde{C}$ und $\widetilde{D}$ von $H^{0}(S, L)$ definiert, für die $\operatorname{mult}\left(\eta \otimes s_{0}+1 \otimes \widetilde{C} s_{0}+\zeta \otimes \widetilde{D} s_{0}\right)=0$ mit $s_{0} \in H^{0}(S, L)$ gilt. Die Wahl einer Basis von $H^{0}(S, L)$ liefert das gesuchte Matrixpaar $(C, D)$ mit Spektralkurve $S$.

\subsection{Ein fester Punkt im Thetadivisor}

Wir nehmen in der gesamten Arbeit an, dass $k>1$ ungerade ist. Dann hat für eine glatte Spektralkurve $S$ das Geradenbündel $\pi_{S}^{*} \mathcal{O}\left(\frac{1}{2}(k-1)\right)$ den Grad $\frac{1}{2} k(k-1)=k+g-1$. Da für $k \geq 3$ das Geradenbündel $\mathcal{O}_{\mathbb{P}^{1}}\left(\frac{1}{2}(k-1)\right) \otimes$ $\mathcal{O}_{\mathbb{P}^{1}}(-1)=\mathcal{O}_{\mathbb{P}^{1}}\left(\frac{1}{2}(k-3)\right)$ über $\mathbb{P}^{1}$ nichttriviale globale holomorphe Schnitte hat, ist die Dimension des Raumes der globalen holomorphen Schnitte des zurückgezogenen Geradenbündels, $h^{0}\left(S, \pi_{S}^{*} \mathcal{O}_{\mathbb{P}^{1}}\left(\frac{1}{2}(k-3)\right)\right)$, größer oder gleich eins. Damit ist $\pi_{S}^{*} \mathcal{O}\left(\frac{1}{2}(k-1)\right) \otimes \pi_{S}^{*} \mathcal{O}(-1) \in W_{g-1} \subset J^{g-1}(S)$ nach dem Riemannschen Singularitätensatz (Satz 1.9) ein singulärer Punkt des Thetadivisors $\Theta=W_{g-1}$, falls $k>3$. Aufgrund der Adjunktionsformel gilt für das kanonische Bündel $K_{S}$ der Kurve $S$ die Gleichung

$$
K_{S}=\left.\left(K_{\widehat{R}} \otimes[S]\right)\right|_{S},
$$

und mit $S=(\widehat{\psi})_{0}$ mit $\widehat{\psi} \in H^{0}\left(\widehat{R},\left[k E_{0}\right]\right)$ und $K_{\widehat{R}}=-2 E_{0}-C$ (siehe $\left.(1.5)\right)$ folgt

$$
K_{S}=\left.\left(\left[-2 E_{0}-C+k E_{0}\right]\right)\right|_{S}=\left.\left(\left[(k-2) E_{\infty}+(k-3) C\right]\right)\right|_{S}=\pi_{S}^{*} \mathcal{O}(k-3) .
$$

Damit ist der Punkt $\pi_{S}^{*} \mathcal{O}\left(\frac{1}{2}(k-1)\right) \otimes \pi_{S}^{*} \mathcal{O}(-1)=\pi_{S}^{*} \mathcal{O}\left(\frac{1}{2}(k-3)\right)$ des Thetadivisors $W_{g-1} \subset J^{g-1}(S)$ eine Thetacharakterisik,

$$
\pi_{S}^{*} \mathcal{O}\left(\frac{1}{2}(k-3)\right) \otimes \pi_{S}^{*} \mathcal{O}\left(\frac{1}{2}(k-3)\right)=K_{S}
$$

Wir wählen diesen Punkt als Ursprung $N:=\pi_{S}^{*} \mathcal{O}\left(\frac{1}{2}(k-3)\right)$ von $J^{g-1}(S)$, d.h. wir identifizieren $J^{g-1}(S)$ mit $J^{0}(S)$ via $L \mapsto L \otimes N^{-1}$, und erhalten so einen Isomorphismus $J^{g-1}(S) \simeq J(S)$.

Definition 1.15. Mit den Konventionen $k>1, k \in \mathbb{N}$ und $k$ ungerade sei ab sofort stets

$$
m:=\frac{1}{2}(k-1)
$$




\section{Kapitel 2}

\section{Grundlegende Berechnungen zur Spektralkurve $S$}

Sei $k \in \mathbb{N}, k$ ungerade und größer als 1 . Sei $(A, B) \in\left(\mathrm{gl}_{k} \times \mathrm{gl}_{k}\right), m=\frac{1}{2}(k-1)$ und $S:=S_{(A, B)}=(\psi)_{0}$ eine glatte Spektralkurve. Das folgende Kapitel besteht aus zwei Teilen. In Abschnitt 2.2 und 2.3 beschreiben wir $H^{1}(S, \mathcal{O})$ und $H^{0}(S, \mathcal{O}(n))$ für beliebige natürliche Zahlen $n<k$ sowie $H^{1}(S, \mathcal{O}(m))$ genauer: Jedes Element $\beta \in H^{1}(S, \mathcal{O})$ kann eindeutig dargestellt werden als $\beta=\sum_{i=2}^{k-1} \eta^{i} \pi^{*} c_{i}$ mit $c_{i} \in H^{1}\left(\mathbb{P}^{1}, \mathcal{O}_{\mathbb{P}^{1}}(-i)\right)$. Für $n<k$ kann jeder Schnitt $s \in$ $H^{0}(S, \mathcal{O}(n))$ eindeutig in der Form $s=\sum_{i=0}^{n} \eta^{i} \pi^{*} c_{i}$ mit $c_{i} \in H^{0}\left(\mathbb{P}^{1}, \mathcal{O}_{\mathbb{P}^{1}}(n-i)\right)$ geschrieben werden. Jedes Element aus $H^{1}(S, \mathcal{O}(m))$ kann eindeutig dargestellt werden als $\sum_{i=2}^{m} \eta^{m+i} \pi^{*} c_{i}$ mit $c_{i} \in H^{1}\left(\mathbb{P}^{1}, \mathcal{O}_{\mathbb{P}^{1}}(-i)\right)$. Hierbei bezeichne $\eta \in H^{0}(S, \mathcal{O}(1))$ jeweils den tautologischen Schnitt.

Im zweiten Teil, den Abschnitten 2.4 und 2.5, gehen wir zunächst auf die Lax-Paar-Bedingung ein. Diese wird durch die Wahl einer Richtung $\beta$ im Tangentialraum $H^{1}(S, \mathcal{O})$ in einem Punkt der Jakobischen festgelegt und gibt eine Bedingung an Äquivalenzklassen von Paaren von Matrizen in $M_{S}$ definiert durch lineare Flüsse in $J^{k+g-1}(S) \backslash \Theta$. In Abschnitt 2.5 zeigen wir, dass alle linearen Flüsse in der Jakobischen, die in Punkten des Thetadivisors starten, diesen sofort verlassen.

\section{$2.1 \quad$ Karten von $S$}

Seien $U_{0}:=\left\{\left(\zeta_{0}: \zeta_{1}\right) \in \mathbb{P}^{1} \mid \zeta_{0} \neq 0\right\}$ und $U_{1}:=\left\{\left(\zeta_{0}: \zeta_{1}\right) \in \mathbb{P}^{1} \mid \zeta_{1} \neq 0\right\}$ die Standardüberdeckungen von $\mathbb{P}^{1}$ mit Karten $\zeta: U_{0} \rightarrow \mathbb{C},\left(\zeta_{0}: \zeta_{1}\right) \mapsto \frac{\zeta_{1}}{\zeta_{0}}$ und $\zeta^{\prime}: U_{1} \rightarrow \mathbb{C},\left(\zeta_{0}: \zeta_{1}\right) \mapsto \frac{\zeta_{0}}{\zeta_{1}}$. Über $U_{0} \cap U_{1}$ gilt $\zeta^{\prime}=\frac{1}{\zeta}$. 
Über $\mathbb{P}^{1}$ sind Vektorbündel und ihre Schnitte mit diesen Karten darstellbar. Für $n \in \mathbb{Z}$ ist das Geradenbündel $\mathcal{O}_{\mathbb{P}^{1}}(n)$ durch die Übergangsfunktion $g_{U_{0} U_{1}}=\zeta^{n}$ über $U_{0} \cap U_{1}$ definiert. Globale holomorphe Schnitte aus dem Raum $H^{0}\left(\mathbb{P}^{1}, \mathcal{O}_{\mathbb{P}^{1}}(n)\right)$ sind eindeutig gegeben durch ihre Repräsentanten, d.h. holomorphe Funktionen $\sum_{i=0}^{n} a_{i} \zeta^{i}$ über $U_{0}$ (und damit über $U_{1}$ durch holomorphe Funktionen $\left.\sum_{i=0}^{n} a_{n-i} \zeta^{-i}=\sum_{i=0}^{n} a_{n-i}\left(\zeta^{\prime}\right)^{i}\right)$. Eine kanonische Basis von $H^{0}\left(\mathbb{P}^{1}, \mathcal{O}(n)\right)$ ist definiert durch die holomorphen Funktionen $\zeta^{j}$ mit $0 \leq j \leq n$ über $U_{0}$.

Genauso können wir $H^{1}\left(\mathbb{P}^{1}, \mathcal{O}(-n)\right)$ beschreiben durch holomorphe Funktionen der Form $\sum_{i=1}^{n-1} a_{i} \zeta^{-i}$ über $U_{0} \cap U_{1}$. Eine kanonische Basis von dem Raum $H^{1}\left(\mathbb{P}^{1}, \mathcal{O}(-n)\right)$ ist definiert durch ihre Repräsentanten $\zeta^{j}$ mit $-n+1 \leq j \leq-1$ über $U_{0} \cap U_{1}$.

Auf ähnliche Weise können wir Schnitte von Geradenbündeln über dem Totalraum $R$ des Geradenbündels $\mathcal{O}_{\mathbb{P}^{1}}(1)$ beschreiben. Seien $\widetilde{U_{0}}:=\pi_{R}^{-1}\left(U_{0}\right)$ und $\widetilde{U_{1}}:=\pi_{R}^{-1}\left(U_{1}\right)$. Der tautologische Schnitt $\eta \in H^{0}(R, \mathcal{O}(1))$ ist dann definiert durch holomorphe Funktionen $\eta: \widetilde{U_{0}} \rightarrow \mathbb{C}$ und $\eta^{\prime}: \widetilde{U_{1}} \rightarrow \mathbb{C}$, die über $\widetilde{U_{0}} \cap \widetilde{U_{1}}$ erfüllen $\eta=\zeta \eta^{\prime}$. Wir erhalten so Karten $\left(\widetilde{U_{0}},(\eta, \zeta)\right)$ bzw. $\left(\widetilde{U_{1}},\left(\eta^{\prime}, \zeta^{\prime}\right)\right)$ von $R$. Mittels Einschränken auf $S \subset R$ erhalten wir schließlich mit $U:=\widetilde{U_{0}} \cap S$ und $U^{\prime}:=\widetilde{U_{1}} \cap S$ Karten für $S$.

\subsection{Der Tangentialraum der Jakobischen}

Die Jakobische $J(S) \simeq H^{1}(S, \mathcal{O}) / H_{1}(S, \mathbb{Z})$ der Spektralkurve $S$ ist ein $g$ dimensionaler Torus. Wir beschreiben im folgenen Satz den Tangentialraum $H^{1}(S, \mathcal{O})$ an die Jakobische in einem Punkt $p \in J(S)$. Die Beweisideen aus ([Hi83], Proposition 3.1) für den Fall von Spektralkurven zu Nahm-Gleichungen werden hier auf unseren Fall übertragen.

Satz 2.1. Jedes Element $\beta \in H^{1}(S, \mathcal{O})$ kann eindeutig dargestellt werden als

$$
\beta=\sum_{i=2}^{k-1} \eta^{i} \pi^{*} c_{i}
$$

mit $c_{i} \in H^{1}\left(\mathbb{P}^{1}, \mathcal{O}_{\mathbb{P}^{1}}(-i)\right)$. Dabei ist $\eta \in H^{0}(S, \mathcal{O}(1))$ der tautologische Schnitt.

\section{Beweis:}

Wir zeigen zunächst $H^{1}\left(\widehat{R},(k-1) E_{\infty}\right) \cong H^{1}(S, \mathcal{O})$. Hierzu betrachten wir die exakte Sequenz

$$
0 \rightarrow \mathcal{O}_{\widehat{R}}\left((k-1) E_{\infty}-k E_{0}\right) \stackrel{\widehat{\psi}}{\rightarrow} \mathcal{O}_{\widehat{R}}\left((k-1) E_{\infty}\right) \rightarrow \mathcal{O}_{S}\left((k-1) E_{\infty}\right) \rightarrow 0
$$


sowie die zugehörige lange exakte Sequenz. Da $E_{0} \cdot E_{\infty}=0$ und $S=(\widehat{\psi})_{0}$ mit $\widehat{\psi} \in H^{0}\left(\widehat{R},\left[k E_{0}\right]\right)$ gilt, ist das Bündel $\mathcal{O}_{S}\left((k-1) E_{\infty}\right)$ trivial, und obige Sequenz hat die Form

$$
0 \rightarrow \mathcal{O}_{\widehat{R}}\left((k-1) E_{\infty}-k E_{0}\right) \rightarrow \mathcal{O}_{\widehat{R}}\left((k-1) E_{\infty}\right) \rightarrow \mathcal{O}_{S} \rightarrow 0 .
$$

Es gilt $(k-1) E_{\infty}-k E_{0}=(k-1) E_{\infty}-k\left(E_{\infty}+C\right)=-E_{\infty}-k C$. Nach Lemma 1.3 ist das Bündel $E_{\infty}+k C$ ampel. Damit folgt nach dem Verschwindungssatz von Kodaira (Satz 1.1):

$$
H^{0}\left(\widehat{R},(k-1) E_{\infty}-k E_{0}\right)=H^{0}\left(\widehat{R},-E_{\infty}-k C\right)=0
$$

und

$$
H^{1}\left(\widehat{R},(k-1) E_{\infty}-k E_{0}\right)=H^{1}\left(\widehat{R},-E_{\infty}-k C\right)=0 .
$$

$\widehat{R}$ ist eine rationale Regelfläche, die Eulercharakteristik $\chi\left(\mathcal{O}_{\widehat{R}}\right)$ ist gleich $1-h^{1}\left(\widehat{R}, \mathcal{O}_{\widehat{R}}\right)+h^{2}\left(\widehat{R}, \mathcal{O}_{\widehat{R}}\right)=1$. Mit dem Satz von Riemann-Roch folgt mit $K_{\widehat{R}}=-2 E_{0}-C=-2 E_{\infty}-3 C$, siehe (1.5),

$$
\begin{aligned}
h^{2}\left(\widehat{R},(k-1) E_{\infty}-k E_{0}\right) & =\chi\left(\mathcal{O}_{\widehat{R}}\right)+\frac{1}{2}\left(\left((k-1) E_{\infty}-k E_{0}\right) .\right. \\
& \left.\quad\left((k-1) E_{\infty}-k E_{0}+2 E_{\infty}+3 C\right)\right) \\
& =1+\frac{1}{2}\left(-(k-1)^{2}-2(k-1)+3(k-1)+k^{2}-3 k\right) \\
& =0 .
\end{aligned}
$$

Somit ist die Einschränkungsabbildung $H^{1}\left(\widehat{R},(k-1) E_{\infty}\right) \rightarrow H^{1}(S, \mathcal{O})$ ein Isomorphismus. Damit ist der erste Teil, $H^{1}\left(\widehat{R},(k-1) E_{\infty}\right) \cong H^{1}(S, \mathcal{O})$, gezeigt.

Sei nun wie in Abschnitt $1.2 \widehat{\xi} \in H^{0}\left(\widehat{R},\left[E_{\infty}\right]\right)$, und sei $\widehat{\eta} \in H^{0}\left(\widehat{R},\left[E_{0}\right]\right)$ die kanonische Fortsetzung vom tautologischen Schnitt $\eta \in H^{0}\left(R, \pi_{R} \mathcal{O}_{\mathbb{P}^{1}}(1)\right)$. Wir definieren

$$
A:=\left\{a=\sum_{i=2}^{k-1} \widehat{\eta}^{i} \widehat{\xi}^{k-1-i} \pi^{*} c_{i} \mid c_{i} \in H^{1}\left(\mathbb{P}^{1}, \mathcal{O}_{\mathbb{P}^{1}}(-i)\right)\right\} \subset H^{1}\left(\widehat{R},\left[(k-1) E_{\infty}\right]\right)
$$

und zeigen $\operatorname{dim} A=h^{1}(S, \mathcal{O})$.

Die Erzeuger des Raumes $A$ sind durch Basen der Räume $H^{1}\left(\mathbb{P}^{1}, \mathcal{O}_{\mathbb{P}^{1}}(-i)\right)$ gegeben. Wir weisen die lineare Unabhängigkeit der Erzeuger von $A$ nach. Angenommen $a:=\sum_{i=2}^{k-1} \widehat{\eta}^{i} \widehat{\xi}^{k-1-i} \pi^{*} c_{i}=0$. Dann gilt insbesondere $\left.a\right|_{E_{\infty}}=0$, und da $\left.\widehat{\xi}\right|_{E_{\infty}}=0$ gilt, folgt $\left.\left(\widehat{\eta}^{k-1} \pi^{*} c_{k-1}\right)\right|_{E_{\infty}}=0 \Rightarrow c_{k-1}=0$. Wir erhalten

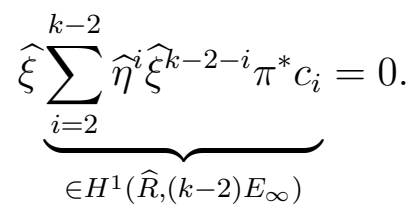


Aus der exakten Sequenz

$$
0 \rightarrow \mathcal{O}_{\widehat{R}}\left((l-1) E_{\infty}\right) \stackrel{\widehat{\xi}}{\rightarrow} \mathcal{O}_{\widehat{R}}\left(l E_{\infty}\right) \rightarrow \underbrace{\mathcal{O}_{E_{\infty}} l\left(E_{0}-C\right)}_{=\mathcal{O}_{E_{\infty}}(-l)} \rightarrow 0, \quad l \in \mathbb{Z},
$$

und der zugehörigen langen exakten Sequenz folgt mit $H^{0}\left(\mathbb{P}^{1}, \mathcal{O}_{\mathbb{P}^{1}}(-l)\right)=0$ für alle $l>0$ die Injektivitiät der Abbildung

$$
\widehat{\xi}: H^{1}\left(\widehat{R},(l-1) E_{\infty}\right) \rightarrow H^{1}\left(\widehat{R}, l E_{\infty}\right)
$$

und damit induktiv $c_{i}=0$ für alle $i=2, \ldots, k-1$. Somit bilden die Basiselemente von $H^{1}\left(\mathbb{P}^{1}, \mathcal{O}_{\mathbb{P}^{1}}(-i)\right)$ eine Basis des Raumes $A$. Es folgt

$$
\begin{aligned}
\operatorname{dim} A & =\sum_{i=2}^{k-1} h^{1}\left(\mathbb{P}^{1}, \mathcal{O}_{\mathbb{P}^{1}}(-i)\right) \\
& =\sum_{i=2}^{k-1}(i-1) \\
& =\frac{1}{2}(k-1)(k-2) \\
& =\operatorname{dim} H^{1}(S, \mathcal{O}) .
\end{aligned}
$$

Folgerung 2.2. Jedes Element $\beta \in H^{1}(S, \mathcal{O})$ kann eindeutig dargestellt werden als

$$
\beta(\eta, \zeta)=\sum_{i=2}^{k-1} \eta^{i} \frac{1}{\zeta^{i}}\left(\sum_{l=1}^{i-1} a_{i l} \zeta^{l}\right)
$$

mit komplexen Zahlen $a_{i l}$.

\subsection{Beschreibung von $H^{i}(S, \mathcal{O}(n))$}

Lemma 2.3. Jeder Schnitt von $H^{0}\left(\widehat{R}, n E_{0}\right), n \in \mathbb{N}$, ist von der Form

$$
s=\sum_{i=0}^{n} \widehat{\eta}^{i} \widehat{\xi}^{n-i} \pi^{*} c_{i}
$$

mit $c_{i} \in H^{0}\left(\mathbb{P}^{1}, \mathcal{O}_{\mathbb{P}^{1}}(n-i)\right)$. 
Beweis: Sei $l \in \mathbb{N}, l \leq n+1$. Wir betrachten die exakte Sequenz von Garben

$$
0 \rightarrow \mathcal{O}_{\widehat{R}}\left((n-l) E_{\infty}+n C\right) \rightarrow \mathcal{O}_{\widehat{R}}\left((n-l+1) E_{\infty}+n C\right) \rightarrow \mathcal{O}_{E_{\infty}}(l-1) \rightarrow 0
$$

und die zugehörige lange exakte Sequenz.

Da das Bündel $(n-l+2) E_{\infty}+(n+3) C$ ampel ist (siehe Lemma 1.3), folgt mit dem Verschwindungssatz von Kodaira (Satz 1.1) $H^{1}\left(\widehat{R},(n-l+2) E_{\infty}+\right.$ $\left.(n+3) C+K_{\widehat{R}}\right)=H^{1}\left(\widehat{R},(n-l) E_{\infty}+n C\right)=0$. Damit folgt

$$
H^{0}\left(\widehat{R},(n-l+1) E_{\infty}+n C\right) \cong H^{0}\left(\mathbb{P}^{1}, \mathcal{O}(l-1)\right) \oplus H^{0}\left(\widehat{R},(n-l) E_{\infty}+n C\right)
$$

und induktiv

$$
\begin{aligned}
H^{0}\left(\widehat{R}, n E_{0}\right) & =H^{0}\left(\widehat{R}, n\left(E_{\infty}+C\right)\right) \\
& =H^{0}\left(\widehat{R},(n-1+1) E_{\infty}+C\right) \\
& =H^{0}\left(\widehat{R},(n-1) E_{\infty}+n C\right) \oplus H^{0}\left(\mathbb{P}^{1}, \mathcal{O}\right) \\
& =\ldots \\
& =\bigoplus_{i=0}^{n} H^{0}\left(\mathbb{P}^{1}, \mathcal{O}_{\mathbb{P}^{1}}(n-i)\right) .
\end{aligned}
$$

Satz 2.4. Für $n<k$ kann jeder Schnitt $s \in H^{0}(S, \mathcal{O}(n))$ eindeutig in der Form

$$
s=\sum_{i=0}^{n} \eta^{i} \pi^{*} c_{i}
$$

mit $c_{i} \in H^{0}\left(\mathbb{P}^{1}, \mathcal{O}_{\mathbb{P}^{1}}(n-i)\right)$ und dem tautologischen Schnitt $\eta \in H^{0}(S, \mathcal{O}(1))$ geschrieben werden.

Beweis: (siehe auch [Hi83] Proposition 4.5)

Wir zeigen, dass die Einschränkungsabbildung $H^{0}\left(\widehat{R}, n E_{0}\right) \rightarrow H^{0}(S, \mathcal{O}(n))$ ein Isomorphismus ist, aus Lemma 2.3 folgt dann die Behauptung. Hierzu betrachten wir die exakte Sequenz

$$
0 \rightarrow \mathcal{O}_{\widehat{R}}\left((n-k) E_{0}\right) \stackrel{\psi}{\rightarrow} \mathcal{O}_{\widehat{R}}\left(n E_{0}\right) \rightarrow \mathcal{O}_{S}(n) \rightarrow 0
$$

und die zugehörige lange exakte Sequenz.

Wegen der Voraussetzung $n-k<0$ ist $H^{0}\left(\widehat{R},(n-k) E_{0}\right)=0$. Es bleibt also $H^{1}\left(\widehat{R},(n-k) E_{0}\right)=0$ zu zeigen. Hierzu betrachten wir für $l \in \mathbb{N}$ die exakte Sequenz

$$
0 \rightarrow \mathcal{O}_{\widehat{R}}\left(-l E_{0}\right) \rightarrow \mathcal{O}_{\widehat{R}}\left((1-l) E_{0}\right) \rightarrow \mathcal{O}_{E_{0}}(1-l) \rightarrow 0
$$


Aufgrund der Rationalität von $\widehat{R}$ ist $H^{1}(\widehat{R}, \mathcal{O})=0$. Für $l=1$ erhalten wir insgesamt die lange exakte Sequenz

$$
0 \rightarrow 0 \rightarrow H^{0}(\widehat{R}, \mathcal{O}) \stackrel{\sim}{\rightarrow} H^{0}\left(E_{0}, \mathcal{O}\right) \rightarrow H^{1}\left(\widehat{R},-E_{0}\right) \rightarrow 0 .
$$

Da $H^{0}(\widehat{R}, \mathcal{O}) \cong H^{0}\left(E_{0}, \mathcal{O}\right)$ gilt, folgt $H^{1}\left(\widehat{R},-E_{0}\right)=0$.

Für $l>1$ ist $H^{0}\left(E_{0}, \mathcal{O}_{E_{0}}(1-l)\right)=0$ und damit die Abbildung

$$
H^{1}\left(\widehat{R},-l E_{0}\right) \rightarrow H^{1}\left(\widehat{R},(1-l) E_{0}\right)
$$

injektiv. Somit wird $H^{1}\left(\widehat{R},-l E_{0}\right)$ für $l>1$ injektiv in $H^{1}\left(\widehat{R},-E_{0}\right)$ abgebildet. Wegen $H^{1}\left(\widehat{R},-E_{0}\right)=0$ folgt für $l:=k-n \geq 1$, dass $H^{1}\left(\widehat{R},-l E_{0}\right)=$ $H^{1}\left(\widehat{R},(n-k) E_{0}\right)=0$ gilt und damit die Behauptung.

Bemerkung 2.5. Wir hätten die globalen holomorphen Schnitte von $\mathcal{O}(n)$ über $S$ auch über die Karten $(U,(\eta, \zeta))$ und $\left(U^{\prime},\left(\eta^{\prime}, \zeta^{\prime}\right)\right)$ berechnen können. Ein Schnitt von $\mathcal{O}_{S}(n)$ ist gegeben durch holomorphe Funktionen $s=\sum_{i, j>0} d_{i j} \eta^{i} \zeta^{j}$ über $U, s^{\prime}=\sum_{i, j>0} d_{i j}^{\prime}\left(\eta^{\prime}\right)^{i}\left(\zeta^{\prime}\right)^{j}$ über $U^{\prime}$, mit $s=\zeta^{n} s^{\prime}$ über $U \cap U^{\prime}$, da $\zeta^{n}=g_{U U^{\prime}}$ die Übergangsfunktion des Bündels $\mathcal{O}_{S}(n)=\pi^{*} \mathcal{O}_{\mathbb{P}^{1}}(n)$ über $S$ ist. Ein Koeffzientenvergleich ergibt die Aussage des Satzes. Die Einschränkung $n<k$ folgt aus der Tatsache, dass über $S$ gilt $\eta^{k}+a_{1} \eta^{k-1}+\ldots+a_{k}=0$.

Für spätere Berechnungen ist eine genauere Beschreibung von $H^{1}(S, \mathcal{O}(m))$ wichtig. Mit dem Satz von Riemann-Roch

$$
\begin{aligned}
h^{0}(S, \mathcal{O}(m))-h^{1}(S, \mathcal{O}(m)) & =1-g+k m \\
& =1-\frac{1}{2}(k-1)(k-2)+\frac{1}{2} k(k-1) \\
& =k
\end{aligned}
$$

folgt aus Satz 2.4

$$
\begin{aligned}
h^{1}(S, \mathcal{O}(m)) & =h^{0}(S, \mathcal{O}(m))-k \\
& =\sum_{i=0}^{\frac{1}{2}(k-1)}\left(\frac{1}{2}(k-1)-i+1\right)-k \\
& =\left(\frac{1}{2}(k-1)+1\right)^{2}-\left(\sum_{i=0}^{\frac{1}{2}(k-1)} i\right)-k \\
& =\frac{1}{8}(k-1)^{2}-\frac{1}{4}(k-1) \\
& =\frac{1}{8}(k-1)(k-3) \\
& =\frac{1}{2} m(m-1) .
\end{aligned}
$$


Damit zeigen wir nun

Satz 2.6. Jedes Element aus $H^{1}(S, \mathcal{O}(m))$ kann eindeutig dargestellt werden als

$$
\sum_{i=2}^{m} \eta^{m+i} \pi^{*} c_{i}
$$

mit $c_{i} \in H^{1}\left(\mathbb{P}^{1}, \mathcal{O}_{\mathbb{P}^{1}}(-i)\right)$.

Beweis: (siehe auch [Hi83] Proposition 5.2)

Wir zeigen zunächst, dass die Einschränkungsabbildung $H^{1}\left(\widehat{R}, m\left(E_{\infty}+E_{0}\right)\right) \rightarrow$ $H^{1}(S, \mathcal{O}(m))$ ein Isomorphismus ist. Dazu betrachten wir die exakte Sequenz

$$
0 \rightarrow \mathcal{O}_{\widehat{R}}\left(m E_{\infty}+(m-k) E_{0}\right) \rightarrow \mathcal{O}_{\widehat{R}}\left(m\left(E_{\infty}+E_{0}\right)\right) \rightarrow \mathcal{O}_{S}(m) \rightarrow 0
$$

sowie die zugehörige lange exakte Sequenz. Es gilt $m E_{\infty}+(m-k) E_{0}=m E_{\infty}+$ $(m-k)\left(E_{\infty}+C\right)=(2 m-k) E_{\infty}+(m-k) C$. Das Bündel $-\left(m E_{\infty}+(m-k) E_{0}\right)=$ $(k-2 m) E_{\infty}+(k-m) C$ ist nach Lemma 1.3 wegen $k-m>k-2 m>0$ ampel. Damit folgt mit dem Verschwindungssatz von Kodaira (Satz 1.1)

$$
H^{0}\left(\widehat{R}, m E_{\infty}+(m-k) E_{0}\right)=H^{1}\left(\widehat{R}, m E_{\infty}+(m-k) E_{0}\right)=0 .
$$

Mit dem Satz von Riemann-Roch und $K_{\widehat{R}}=-2 E_{0}-C$ erhalten wir

$$
\begin{aligned}
h^{2}\left(\widehat{R}, m E_{\infty}+(m-k) E_{0}\right)= & \chi\left(\mathcal{O}_{\widehat{R}}\right)+\frac{1}{2}\left(m E_{\infty}+(m-k) E_{0}\right) \\
& \cdot\left(m E_{\infty}+(m-k) E_{0}+2 E_{0}+C\right) \\
= & 1+\frac{1}{2}\left(-m^{2}+m+(m-k)^{2}+2(m-k)+m-k\right) \\
= & 1+\frac{1}{2}\left(k^{2}-2 m k+4 m-3 k\right) \\
= & 1+\frac{1}{2}\left(k^{2}-(k-1) k+2 k-2-3 k\right) \\
= & 0 .
\end{aligned}
$$

Somit ist die Einschränkungsabbildung $H^{1}\left(\widehat{R}, m\left(E_{\infty}+E_{0}\right)\right) \rightarrow H^{1}(S, \mathcal{O}(m))$ ein Isomorphismus.

Wir zeigen nun, dass mit $\widehat{\eta} \in H^{0}\left(\widehat{R},\left[E_{0}\right]\right)$ und $\widehat{\xi} \in H^{0}\left(\widehat{R},\left[E_{\infty}\right]\right)$ die Dimension des Unterraumes

$$
A:=\left\{a=\sum_{i=2}^{m} \widehat{\eta}^{m+i} \widehat{\xi}^{m-i} \pi^{*} c_{i} \mid c_{i} \in H^{1}\left(\mathbb{P}^{1}, \mathcal{O}_{\mathbb{P}^{1}}(-i)\right)\right\}
$$


von $H^{1}\left(\widehat{R}, m\left(E_{\infty}+E_{0}\right)\right)$ gleich $h^{1}(S, \mathcal{O}(m))=\frac{1}{2} m(m-1)$ ist, und damit die Behauptung.

Die Erzeuger des Raumes $A$ sind durch Basen der Räume $H^{1}\left(\mathbb{P}^{1}, \mathcal{O}_{\mathbb{P}^{1}}(-i)\right)$ gegeben. Wir weisen die lineare Unabhängigkeit der Erzeuger nach. Angenommen $a=\sum_{i=2}^{m} \widehat{\eta}^{m+i} \widehat{\xi}^{m-i} \pi^{*} c_{i}=0$. Dann ist insbesondere auch $\left.a\right|_{E_{\infty}}=0$, d.h. es folgt $c_{m}=0$. Wir erhalten

$$
\underbrace{\widehat{\xi} \sum_{i=2}^{m-1} \widehat{\eta}^{m+i} \widehat{\xi}^{m-i-1} \pi^{*} c_{i}}_{\in H^{1}\left(\widehat{R},(m-1) E_{\infty}+m E_{0}\right)}=0 .
$$

Mit der exakten Sequenz

$0 \rightarrow \mathcal{O}_{\widehat{R}}\left((l-1) E_{\infty}+m E_{0}\right) \stackrel{\widehat{\xi}}{\rightarrow} \mathcal{O}_{\widehat{R}}\left(l E_{\infty}+m E_{0}\right) \rightarrow \underbrace{\mathcal{O}_{E_{\infty}}\left(l\left(E_{0}-C\right)+m E_{0}\right)}_{=\mathcal{O}_{E_{\infty}}(-l)} \rightarrow 0$

folgt mit $H^{0}\left(\mathbb{P}^{1}, \mathcal{O}_{\mathbb{P}^{1}}(-l)\right)=0$ für alle $l>0$ die Injektivitiät der Abbildung $\widehat{\xi}: H^{1}\left(\widehat{R},(l-1) E_{\infty}+m E_{0}\right) \rightarrow H^{1}\left(\widehat{R}, l E_{\infty}+m E_{0}\right)$ und damit induktiv $c_{i}=0$ für alle $i=2, \ldots, m$. Wir erhalten

$$
\begin{aligned}
\operatorname{dim} A & =\sum_{i=2}^{m} h^{1}\left(\mathbb{P}^{1}, \mathcal{O}_{\mathbb{P}^{1}}(-i)\right) \\
& =\sum_{i=2}^{m}(i-1) \\
& =\frac{1}{2} m(m-1) \\
& =h^{1}(S, \mathcal{O}(m)) .
\end{aligned}
$$

\subsection{Lax-Paar-Bedingung}

Wir betrachten in diesem Abschnitt nur lineare Flüsse in $J^{k+g-1}(S) \backslash \Theta$. Sei $\beta \in H^{1}(S, \mathcal{O})$ und $\mathrm{L} \in J^{k+g-1} \backslash \Theta$ ein Geradenbündel mit Übergangsfunktion $l:=l_{U U^{\prime}}$. Die Übergangsfunktionen $l \exp (z \beta)$ definieren eine Familie $L^{z} \otimes \mathbf{L}$ von Geradenbündeln über $S$ vom Grad $k+g-1$ mit $L^{z} \otimes \mathrm{L} \in J^{k+g-1}(S) \backslash \Theta$ für allgemeines $z \in \mathbb{C}$. Sei $M \rightarrow \mathbb{C} \times S$ das Geradenbündel definiert durch $\left.M\right|_{(z, w)}=$ $\left.\left(L^{z} \otimes \mathrm{L}\right)\right|_{w},(z, w) \in \mathbb{C} \times S$. Die direkte Bildgarbe $\pi_{*} M$ mit $\pi: \mathbb{C} \times S \rightarrow \mathbb{C}$ ist 
über $\mathbb{C}$ torsionsfrei, aufgrund des Struktursatzes für endlich erzeugte Moduln also eine lokal freie Garbe. Somit definiert $\pi_{*} M$ ein Vektorbündel $V$ über $\mathbb{C}$ vom Rang $k$ mit Faser $V_{z}=H^{0}\left(S, L^{z} \otimes \mathrm{L}\right)$ für allgemeines $z$, d.h. für $z$ mit $L^{z} \otimes \mathrm{L} \notin \Theta$.

Wir zeigen

Satz 2.7. Für jeden linearen Fluß $L^{z} \otimes \mathbf{L}$ in der Jakobischen $J^{k+g-1}(S) \backslash \Theta$ gibt es in einer hinreichend kleinen Umgebung $\mathcal{U}$ von $0 \in \mathbb{C}$ eine Familie von Repräsentanten $(A(z), B(z))$ der Urbilder von $L^{z} \otimes \mathrm{L}$ in $M_{S} \subset\left(g l_{k} \times g l_{k}\right) / G L_{k}$, die die Lax-Paar-Bedingung $\frac{d}{d z} \mathcal{C}=[\mathcal{C}, D]$ mit $\mathcal{C}=\mathcal{C}(z)=A(z)+\zeta B(z)$ und $D=\beta(\mathcal{C}(z), \zeta)^{+}$erfüllen. Hierbei bezeichne $\beta(\mathcal{C}(z), \zeta)^{+}$den polynomialen Anteil von $\beta(\mathcal{C}(z), \zeta)$ in $\zeta$.

Beweis: ([AHH90]) Sei $\widehat{\mathcal{U}}$ eine Umgebung von $0 \in \mathbb{C}$, in der für alle $z \in \widehat{\mathcal{U}}$ das Geradenbündel $L^{z} \otimes \mathrm{L}$ nicht im Thetadivisor $\Theta \subset J^{k+g-1}(S)$ liegt. Die Urbilder von $L^{z} \otimes \mathrm{L}$ in $M_{S} \subset\left(\mathrm{gl}_{k} \times \mathrm{gl}_{k}\right) / \mathrm{GL}_{k}$ sind nach Satz 1.14, für $z \in \widehat{\mathcal{U}}$ definiert durch die Endomorphismen $\widehat{A(z)}, \widehat{B(z)}$ von $V_{z}=H^{0}\left(S, L^{z} \otimes \mathrm{L}\right)$ mit $(\eta+\widetilde{A(z)}+\zeta \widetilde{B(z)}) \sigma(z)=0$ für alle $\sigma(z) \in V_{z}$. Hierbei ergibt für festes $z$ die Wahl einer Basis der Faser $V_{z}$ ein Paar von Matrizen $(A(z), B(z))$ mit Spektralkurve $S$. Die Matrizen sind nur abhängig von der Wahl der Basis, d.h. modulo Konjugation mit Matrizen $X(z) \in \mathrm{GL}_{k}$ eindeutig bestimmt.

Wir konstruieren im Folgenden eine Rahmung des Vektorbündels $V \rightarrow \mathbb{C}$, d.h. eine Familie von Basen $\sigma_{1}(z), \ldots \sigma_{k}(z)$ von $V_{z}$, die holomorph von $z$ abhängt und für die der Fluß $(A(z), B(z)) \in \mathrm{gl}_{k} \times \mathrm{gl}_{k}$ die Lax-Paar-Bedingung $\frac{d}{d z} \mathcal{C}=[\mathcal{C}, D]$ erfüllt. Diese spezielle Rahmung definieren wir durch kovariant konstante Schnitte bezüglich eines noch zu definierenden Zusammenhangs des Vektorbündels $V \rightarrow \mathbb{C}$.

Sei $\sigma$ ein Schnitt von $V$ über $\widehat{\mathcal{U}}$. Jedes Element $\sigma(z) \in V_{z}=H^{0}\left(S, L^{z} \otimes \mathbf{L}\right)$ wird durch holomorphe Funktionen $s(z): U \rightarrow \mathbb{C}$ und $s^{\prime}(z): U^{\prime} \rightarrow \mathbb{C}$ mit $s(z)=l \exp (z \beta(\eta, \zeta)) s^{\prime}(z)$ über $U \cap U^{\prime}$ definiert. Ableiten nach $z$ ergibt über $U \cap U^{\prime}$ :

$$
\begin{aligned}
\frac{\partial s}{\partial z} & =\beta(\eta, \zeta) l \exp (z \beta(\eta, \zeta)) s^{\prime}(z)+l \exp (z \beta(\eta, \zeta)) \frac{\partial s^{\prime}}{\partial z} \\
& =\beta(\eta, \zeta) s(z)+l \exp (z \beta(\eta, \zeta)) \frac{\partial s^{\prime}}{\partial z} \\
& =\left.\beta(-(\widetilde{A}+\zeta \widetilde{B}), \zeta) \sigma\right|_{U \cap U^{\prime}}+l \exp (z \beta(\eta, \zeta)) \frac{\partial s^{\prime}}{\partial z}
\end{aligned}
$$

da $\eta \sigma=-(\widetilde{A}+\zeta \widetilde{B}) \sigma$ gilt. Sei $\beta(-(\widetilde{A}+\zeta \widetilde{B}), \zeta)^{+}$der polynomiale Anteil von $\beta(-(\widetilde{A}+\zeta \widetilde{B}), \zeta)$ abhängig von $\zeta$, entsprechend $\beta(-(\widetilde{A}+\zeta \widetilde{B}), \zeta)^{-}$der Laurent- 
Hauptteil von $\beta(-(\widetilde{A}+\zeta \widetilde{B}), \zeta)$ abhängig von $\zeta$. Dann gilt über $U \cap U^{\prime}$ :

$$
\begin{aligned}
\frac{\partial s}{\partial z}-\beta(-(\widetilde{A}+\zeta \widetilde{B}), \zeta)^{+} s & =\beta(-(\widetilde{A}+\zeta \widetilde{B}), \zeta)^{-} s+l \exp (z \beta(\eta, \zeta)) \frac{\partial s^{\prime}}{\partial z} \\
& =\beta(-(\widetilde{A}+\zeta \widetilde{B}), \zeta)^{-} l \exp (z \beta(\eta, \zeta)) s^{\prime} \\
& +l \exp (z \beta(\eta, \zeta)) \frac{\partial s^{\prime}}{\partial z} \\
& =l \exp (z \beta(\eta, \zeta))\left(\frac{\partial s^{\prime}}{\partial z}+\beta(-(\widetilde{A}+\zeta \widetilde{B}), \zeta)^{-} s^{\prime}\right) .
\end{aligned}
$$

Hierbei ist $\frac{\partial s}{\partial z}-\beta(-(\widetilde{A}+\zeta \widetilde{B}), \zeta)^{+} s$ holomorph in $\zeta$ und $\frac{\partial s^{\prime}}{\partial z}+\beta(-(\widetilde{A}+\zeta \widetilde{B}), \zeta)^{-} s^{\prime}$ holomorph in $\zeta^{-1}$. Wir erhalten also einen wohldefinierten Zusammenhang $\nabla_{z}$ von $V$ über $\widehat{\mathcal{U}}$ mit

$$
\left.\nabla_{z} \sigma\right|_{U}=\frac{\partial s}{\partial z}-\left.\beta(-(\widetilde{A}+\zeta \widetilde{B}), \zeta)^{+} s\right|_{U}
$$

bzw.

$$
\left.\nabla_{z} \sigma\right|_{U^{\prime}}=\frac{\partial s^{\prime}}{\partial z}+\left.\beta(-(\widetilde{A}+\zeta \widetilde{B}), \zeta)^{-} s^{\prime}\right|_{U^{\prime}}
$$

Es gibt nun eine (evtl. kleinere) Umgebung $\mathcal{U} \subset \widehat{\mathcal{U}}$ von 0 und Schnitte $\sigma_{1}, \ldots, \sigma_{k} \in \Gamma\left(\mathcal{U},\left.V\right|_{\mathcal{U}}\right)$, so dass für alle $z \in \mathcal{U}$ die Elemente $\sigma_{1}(z), \ldots, \sigma_{k}(z)$ eine Basis von $V_{z}$ bilden und kovariant konstant in $\mathcal{U}$ sind. Sei $\widetilde{\mathcal{C}}:=\widetilde{A}+\zeta \widetilde{B}$ und $\mathcal{C}=A+\zeta B$ die Matrixdarstellung von $\widetilde{\mathcal{C}}$ bezüglich der Basis $\sigma_{1}, \ldots, \sigma_{k}$.

Sei $\sigma \in \Gamma\left(\mathcal{U},\left.V\right|_{\mathcal{U}}\right)$ ein kovariant konstanter Schnitt. Wegen $(\eta+\mathcal{C}) \sigma=0$ folgt dann $(\eta+\mathcal{C}) \frac{\partial \sigma}{\partial z}+\frac{\partial \mathcal{C}}{\partial z} \sigma=0$. Da $\sigma$ kovariant konstant in $\mathcal{U}$ ist, gilt $\frac{\partial \sigma}{\partial z}-\beta(-\mathcal{C}, \zeta)^{+} \sigma=0$ über $U$. Wir erhalten über $U$ :

$$
\begin{aligned}
0 & =(\eta+\mathcal{C}) \beta^{+}(-\mathcal{C}, \zeta) \sigma+\frac{\partial \mathcal{C}}{\partial z} \sigma \\
& =\beta^{+}(-\mathcal{C}, \zeta) \eta \sigma+\mathcal{C} \beta^{+}(-\mathcal{C}, \zeta) \sigma+\frac{\partial \mathcal{C}}{\partial z} \sigma \\
& =-\beta^{+}(-\mathcal{C}, \zeta) \mathcal{C} \sigma+\mathcal{C} \beta^{+}(-\mathcal{C}, \zeta) \sigma+\frac{\partial \mathcal{C}}{\partial z} \sigma \\
& =\left(\left[\mathcal{C}, \beta^{+}(-\mathcal{C}, \zeta)\right]+\frac{\partial \mathcal{C}}{\partial z}\right) \sigma .
\end{aligned}
$$

Wir zeigen nun $\frac{\partial \mathcal{C}}{\partial z}=-\left[\mathcal{C}, \beta^{+}(-\mathcal{C}, \zeta)\right]$ :

Sei $z_{0} \in \mathcal{U}$ fest. Sei $C \subset R$ eine Faser, so dass der Schnitt $S \cap C$ aus $k$ verschiedenen Punkten $\left\{x_{1}, \ldots, x_{k}\right\}$ besteht. Dies entspricht $k$ verschiedenen Eigenwerten zur festen Matrix $\left(\zeta_{0} A+\zeta_{1} B\right)$ mit festem $\left(\zeta_{0}: \zeta_{1}\right) \in \mathbb{P}^{1}$, wobei 
wir ohne Einschränkung $\left(\zeta_{0}: \zeta_{1}\right) \in U_{0}$ annehmen. $C$ ist der Divisor eines Schnittes $\delta \in H^{0}\left(R, \pi_{S}^{*} \mathcal{O}_{\mathbb{P}^{1}}(1)\right)$. Aus der exakten Sequenz

$$
0 \rightarrow \mathcal{O}_{S}\left(L^{z_{0}} \otimes \mathbf{L}\right) \otimes \pi_{S}^{*} \mathcal{O}_{\mathbb{P}^{1}}(-1) \stackrel{\delta}{\rightarrow} \mathcal{O}_{S}\left(L^{z_{0}} \otimes \mathbf{L}\right) \rightarrow \mathcal{O}_{S \cap C} \rightarrow 0
$$

erhalten wir mit der Voraussetzung $L^{z_{0}} \otimes \mathbf{L} \notin \Theta \subset J^{k+g-1}(S)$ und damit $H^{0}\left(S, L^{z_{0}} \otimes \mathrm{L}(-1)\right)=0$ und $H^{1}\left(S, L^{z_{0}} \otimes \mathrm{L}(-1)\right)=0$, dass die Einschränkungsabbildung

$$
H^{0}\left(S, L^{z_{0}} \otimes L\right) \rightarrow H^{0}(S \cap C, \mathcal{O})=H^{0}\left(\left\{x_{1}, \ldots, x_{k}\right\}, \mathcal{O}\right)
$$

ein Isomorphismus ist. Damit gibt es also eine Basis $b_{1}, \ldots, b_{k}$ von $H^{0}\left(S, L^{z_{0}} \otimes\right.$ $L)$ mit $b_{i}\left(x_{j}\right)=0$ falls $i \neq j$ und $b_{i}\left(x_{j}\right) \neq 0$ falls $i=j$. Bezeichne $M$ die Matrix $\left(\left[\mathcal{C}, \beta^{+}\right]+\frac{\partial \mathcal{C}}{\partial z}\right)$. Dann folgt $\sum_{j=1}^{k} M_{i j} b_{j}\left(x_{l}\right)=0$ für alle $l, i$ und damit $M_{i j}=0$ für alle $i, j$. Damit erhalten wir $\frac{\partial \mathcal{C}}{\partial z}=-\left[\mathcal{C}, \beta^{+}(-\mathcal{C}, \zeta)\right]$.

Bemerkung 2.8. Verwenden wir statt einer Rahmung $\sigma_{1}(z), \ldots, \sigma_{k}(z)$ von kovariant konstanten Schnitten eine beliebige Rahmung $\sigma_{1}^{\prime}(z), \ldots \sigma_{k}^{\prime}(z)$ über $\mathcal{U}$, so gilt - falls $\mathcal{C}$ die Matrixdarstellung von $\widetilde{\mathcal{C}}$ bezüglich der $\sigma_{i}^{\prime}$ und $N_{z}$ die Matrixdarstellung von $\nabla_{z}$ bezüglich der $\sigma_{i}^{\prime}$ ist - lokal

$$
\begin{aligned}
0 & =(\eta+\mathcal{C}) \beta^{+}(-\mathcal{C}, \zeta) \sigma_{i}^{\prime}+\frac{\partial \mathcal{C}}{\partial z} \sigma_{i}^{\prime}+(\eta+\mathcal{C}) N_{z} \sigma_{i}^{\prime} \\
& =\beta^{+}(-\mathcal{C}, \zeta) \eta \sigma_{i}^{\prime}+\mathcal{C} \beta^{+}(-\mathcal{C}, \zeta) \sigma_{i}^{\prime}+\frac{\partial \mathcal{C}}{\partial z} \sigma_{i}^{\prime}+(\eta+\mathcal{C}) N_{z} \sigma_{i}^{\prime} \\
& =-\beta^{+}(-\mathcal{C}, \zeta) \mathcal{C} \sigma_{i}^{\prime}+\mathcal{C} \beta^{+}(-\mathcal{C}, \zeta) \sigma_{i}^{\prime}+\left(\frac{\partial \mathcal{C}}{\partial z}+\left[\mathcal{C}, N_{z}\right]\right) \sigma_{i}^{\prime} \\
& =\left(\left[\mathcal{C}, \beta^{+}(-\mathcal{C}, \zeta)\right]+\frac{\partial \mathcal{C}}{\partial z}+\left[\mathcal{C}, N_{z}\right]\right) \sigma_{i}^{\prime} .
\end{aligned}
$$

Bemerkung 2.9. Die Anwendung von Resultaten dieser Arbeit auf Hitchins Spektralkurve zu den Nahm-Gleichungen ist eine Untersuchung wert: Ist (wie in Kapitel 3) $\beta=\frac{\eta^{2}}{\zeta}$ und erfüllt $\mathcal{C}(z):=A(z)+\zeta B(z)$ die Lax-Paar-Bedingung $\dot{\mathcal{C}}=\left[\mathcal{C}, \beta(\mathcal{C}, \zeta)^{+}\right]$, so beschreibt $\mathcal{C}(z)^{2}$ Hitchins Spektralkurve ([Hi83]) zu den Nahmgleichungen, d.h. $\mathcal{C}(z)^{2}$ erfüllt die Lax-Paar-Bedingung

$$
\left(C_{0}+\zeta C_{1}+\zeta^{2} C_{2}\right)^{\prime}=\left[C_{0}+\zeta C_{1}+\zeta^{2} C_{2}, C_{1}+\zeta C_{2}\right]
$$

mit $C_{0}:=A^{2}, C_{1}:=A B+B A$ und $C_{2}:=B^{2}$. 


\subsection{Isospektrale Flüsse durch den Thetadivi- sor}

Wir haben bisher isospektrale Flüsse in $J^{k+g-1}(S) \backslash \Theta$ betrachtet. Für die spätere Darstellung von Funktionen mithilfe von Thetafunktionen ist gerade das Verhalten in Punkten des Thetadivisors interessant. In diesem Abschnitt geht es darum, dass jeder Fluss $L^{z} \otimes L \in J^{k+g-1}(S)$ mit $L^{z}$ definiert durch ein $\beta \in H^{1}(S, \mathcal{O})$ und $\mathbf{L} \in \Theta$ den Thetadivisor sofort verlässt, d.h. $L^{z} \otimes \mathbf{L} \notin \Theta$ für $z \neq 0,|z|$ hinreichend klein.

Der Tangentialraum $T_{p} \Theta$ in einem Punkt $p \in \Theta$ ist durch die Nullstellenmenge von

$$
\sum_{i=1}^{g} \frac{\partial \vartheta}{\partial z_{i}}(p)\left(z_{i}-z_{i}(p)\right)
$$

gegeben. Hierbei bezeichnet $\vartheta$ die Riemannsche Thetafunktion. Die Gaussabbildung $G: \Theta_{\text {glatt }} \rightarrow \mathbb{P}^{g-1}$, die durch

$$
p \mapsto\left(\frac{\partial \vartheta}{\partial z_{1}}(p): \ldots: \frac{\partial \vartheta}{\partial z_{g}}(p)\right)
$$

definiert ist, bildet einen glatten Punkt des Thetadivisors auf seinen zugehörigen Tangentialraum ab. Da das Bild der Gaussabbildung in keiner Hyperebene enthalten ist (siehe [BL04] Proposition 4.4.1), ist der Schnitt aller in den Ursprung verschobenen Tangentialräume an nichtsingulären Punkten von $\Theta$ leer, d.h. jedes beliebige lineare Vektorfeld $V$ von $J^{g-1}(S)$ schneidet den Thetadivisor $\Theta \in J^{g-1}(S)$ im allgemeinen Punkt transversal. In diesen Punkten $L$ folgt sofort, dass $L^{z} \otimes \mathbf{L}$ für $z \neq 0,|z|$ hinreichend klein, nicht mehr in $\Theta$ liegt.

Angenommen, für einen Startpunkt $L \in \Theta$ verläuft der lineare Fluss $L^{z} \otimes \mathbf{L}$ für $z \in \mathcal{U}$ mit einer Umgebung $\mathcal{U}$ von $0 \in \mathbb{C}$ im Thetadivisor. Dann enthält der Thetadivisor den gesamten Fluss $L^{z} \otimes \mathrm{L}$. Verschieben wir $\Theta$ entlang dieses Flusses $2^{2 g}$ mal so, dass jeweils der verschobene Divisor durch eine Thetacharakteristik verläuft, so erhalten wir $2^{2 g}$ Geradenbündel, die eine Einbettung der Jakobischen in den projektiven Raum definieren. Jedoch liegt nach Konstruktion die Gerade $g$ in der Basismenge für jedes der neuen Geradenbündel. Dies ist ein Widerspruch dazu, dass sie eine Einbettung definieren und damit die Schnitte nicht in allen Punkten gleichzeitig verschwinden können. 


\section{Kapitel 3}

\section{Painlevé-Analysis}

Sei wie bisher $k$ eine natürliche ungerade Zahl größer als eins und $(A, B)$ ein Element von $\left(\mathrm{gl}_{k} \times \mathrm{gl}_{k}\right) / G L_{k}$ mit glatter Spektralkurve $S:=S_{(A, B)}$, und sei $M_{S}$ die Menge aller Äquivalenzklassen von Paaren von Matrizen mit Spektralkurve $S$. Mit dem Satz von Beauville (Satz 1.13) wissen wir, dass $M_{S}$ mit der Jakobischen von $S$ ohne dem Thetadivisor identifiziert werden kann, $M_{S} \cong J(S) \backslash \Theta$. Diese Identifizierung nutzen wir, um Funktionen auf $M_{S}$ mittels Thetafunktionen auszudrücken:

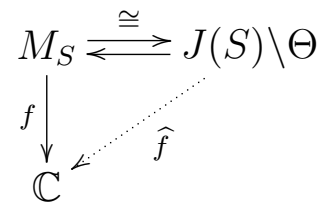

Eine holomorphe Funktion $f: M_{S} \rightarrow \mathbb{C}$ entspricht einer meromorphen Funktion $\widehat{f}: J(S) \rightarrow \mathbb{C}$, die genau in $\Theta$ Polstellen hat. Die Menge aller meromorphen Funktionen mit Polstellen im Thetadivisor ist in Abschnitt 1.3 beschrieben. Jede solche Funktion $\widehat{f}$ läßt sich durch Thetafunktionen mittels $\widehat{f}=\frac{g}{\vartheta^{n}}$ mit $g \in R_{n}^{Z}, n \in \mathbb{N}$, darstellen, siehe hierzu Satz 1.10. Zur exakten Bestimmung von $\widehat{f}$ muss die Art der Polstellen in Punkten von $\Theta$ untersucht werden. Auf den genauen Algorithmus gehen wir in Kapitel 6 ein. Wir nutzen dabei die Tatsache, dass das Verhalten von $\widehat{f}$ in einem allgemeinen Punkt $p$ des Thetadivisors eindeutig durch das Verhalten von $\widehat{f}$ entlang eines zu $\Theta$ transversalen linearen Flusses in $J(S)$ durch $p$ bestimmt ist. Dies folgt aus

Satz 3.1. [AMV04] Sei $\mathcal{V}$ ein holomorphes Vektorfeld auf der Jakobischen $J(S)$ definiert durch $\beta \in H^{1}(S, \mathcal{O})$. Dann gibt es für jeden allgemeinen Punkt $p \in \Theta$ (d.h. für jeden glatten Punkt $p \in \Theta$, in dem $\mathcal{V}$ transversal zu $\Theta$ ist) eine Koordinatenumgebung $p \in U \subset J(S)$ mit Koordinaten $\left(t, x_{2}, \ldots, x_{g}\right)$ und 
eine Umgebung $p \in V \subset \Theta$ von $p$ mit Koordinaten $\left(x_{2}, \ldots, x_{g}\right)$ in $\Theta$, so dass $\mathcal{V}=\frac{\partial}{\partial t}$ über $U$ gilt.

Wir bestimmen in diesem Kapitel das asymptotische Verhalten einer Familie von Äquivalenzklassen von Paaren $(A(z), B(z))$, für die die zugehörigen Geradenbündel entlang eines festen isospektralen Flusses in der Jakobischen definiert durch eine Richtung $\beta \in H^{1}(S, \mathcal{O})$ gegen einen allgemeinen Punkt im Thetadivisor laufen. Hierzu verwenden wir Painlevé-Analysis.

\subsection{Grundlegende Prinzipien}

Wir fassen zunächst für uns wichtige Begriffe der Painlevé-Analysis zusammen. Details findet man zum Beispiel in [AMV04] und in [V08]. Sei

$$
\dot{x}=f(x)
$$

eine Differentialgleichung in $\mathbb{C}^{n}$, wobei die Komponenten $f_{i}$ von $f=\left(f_{1}, \ldots, f_{n}\right)$ Polynome in $x_{1}, \ldots, x_{n}$ seien.

Definition 3.2. ([AMV04] Definition 6.6) Ein n-Tupel von Laurentreihen $x_{i}(z)=\frac{1}{z^{r_{i}}} \sum_{k=0}^{\infty} x_{i}^{(k)} z^{k}$ mit $i=1, \ldots, n$ und ganzen Zahlen $r_{i} \in \mathbb{Z}$ heißt formale Laurentlösung von $\dot{x}=f(x)$, falls die $x_{i}(z)$ eingesetzt in die Differentialgleichung (3.1) eine formale Gleichheit von Reihen ergeben. Gibt es ein $\epsilon>0$, so dass die formalen Laurentreihen $\left(x_{1}(z), \ldots, x_{n}(z)\right)$ für $0<|z|<\epsilon$ konvergieren, so sprechen wir von konvergenten Laurentlösungen. Ist $x_{i}^{(0)} \neq 0$, so hat $x_{i}(z)$ für positives $r_{i}$ einen Pol der Ordnung $r_{i}$ bzw. für negatives $r_{i}$ eine Nullstelle der Ordnung $\left|r_{i}\right|$. Ist $r:=\max \left\{r_{i}\right\}>0$, so sprechen wir von einer strikten Laurentlösung mit Polordnung $r$.

Für jeden beliebigen Startpunkt $p \in \mathbb{C}^{n}$ existiert nach dem Satz von PicardLindelöf für gewöhnliche Differentialgleichungen eine eindeutige Lösung von (3.1). Diese definiert eine konvergente Taylorreihe und damit eine konvergente (nicht strikte) Laurentlösung. Die Menge all dieser Taylorlösungen wird durch die Startpunkte $p \in \mathbb{C}^{n}$ parametrisiert. Analog zu den Taylorlösungen können wir auch strikte Laurentlösungen von (3.1) zu Familien zusammenfassen. Diese Familien werden nicht mehr durch Teilmengen von $\mathbb{C}^{n}$, sondern durch (in unserem Fall algebraische) Mengen $\left(\nsubseteq \mathbb{C}^{n}\right)$ parametrisiert.

Definition 3.3. ([AMV04] Definition 6.7) Eine Familie $x(z)$ von strikten formalen Laurentlösungen von (3.1), die durch eine irreduzible Komponente einer algebraischen Menge parametrisiert wird, heißt Balance. Die Dimension der irreduziblen Komponente bestimmt hierbei die Anzahl der Parameter, 
von denen die formalen Laurentlösungen $x(z)$ abhängen. Ist diese Dimension gleich $n-1$, so sprechen wir von einer Hauptbalance, ansonsten von einer niedrigeren Balance.

Wir haben zu Beginn dieses Abschnittes eine nichtsinguläre Spektralkurve fixiert. Eine Richtung $\beta \in H^{1}(S, \mathcal{O})$ definiert ein lineares Vektorfeld auf der Jakobischen $J(S)$ und damit die Lax-Paar-Gleichung $\dot{\mathcal{C}}=[\mathcal{C}, D]$ (siehe Satz 2.7), wobei diese eine Differentialgleichung auf ganz $\left(\mathrm{gl}_{k} \times \mathrm{gl}_{k}\right) / \mathrm{GL}_{k}$ definiert. Statt einer festen Spektralkurve betrachten wir nun eine Familie von Spektralkurven und damit eine Familie von Jakobischen, auf die sich das durch $\beta$ definierte Vektorfeld fortsetzen läßt.

Satz 3.4. ([AMV04] Theorem 6.9) Sei $F: \mathbb{C}^{n} \rightarrow \mathbb{C}^{s}$ eine polynomiale $A b$ bildung, so dass für einen allgemeinen Punkt $c \in \mathbb{C}^{s}$ ein Isomorphismus $j_{c}: F^{-1}(c) \rightarrow T_{c}^{r} \backslash D_{c}$ mit einer irreduziblen Abelschen Varietät $T_{c}^{r}$ der Dimension $r=n-s$ und einer analytischen Hyperfläche $D_{c}$ von $T_{c}^{r}$ existiert. Ist $\mathcal{V}$ ein lineares Vektorfeld auf $\mathbb{C}^{n}$, welches sich für allgemeines $c$ auf ein lineares Vektorfeld auf ganz $T_{c}^{r}$ fortsetzen lässt, so gibt es für ein allgemeines $c_{0} \in \mathbb{C}^{s}$

(a) eine Umgebung $U \subset \mathbb{C}^{s}$ von $c_{0}$,

(b) eine nichtsinguläre algebraische Varietät $M(U)$,

(c) eine surjektive Abbildung $\pi: M(U) \rightarrow U$,

(d) eine Einbettung $\iota: F^{-1}(U) \rightarrow M(U)$ und

(e) eine analytische Hyperfläche $D$ von $M(U)$,

so dass

1. das Diagramm

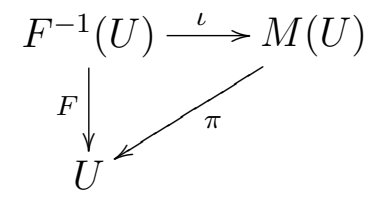

kommutiert,

2. $\iota: F^{-1}(U) \rightarrow M(U) \backslash D$ ein Isomorphismus ist und 
3. es für jedes $c \in U$ einen Isomorphismus $\overline{\iota_{c}}: \pi^{-1}(c) \rightarrow T_{c}^{r}$ gibt, so dass

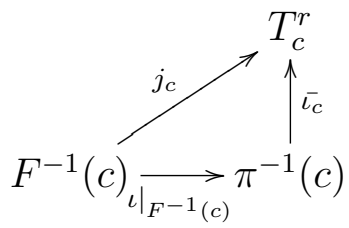

kommutiert.

$M(U)$ besitzt ein holomorphes Vektorfeld $\overline{\mathcal{V}}$, welches ı-bezogen zur Einschränkung von $\mathcal{V}$ auf $F^{-1}(U)$ ist.

Bemerkung 3.5. [AMV04] Satz 3.4 gilt auch mit der schwächeren Voraussetzung, dass $T_{c}^{r}$ für einen allgemeinen Punkt $c \in \mathbb{C}^{s}$ eine (unter Umständen nicht mehr irreduzible) Abelsche Varietät ist und Integralkurven zu $\mathcal{V}$, die in $D_{c}$ starten, den Divisor $D_{c}$ sofort verlassen.

Haben wir für eine feste, nichtsinguläre Spektralkurve $S_{0}$ eine Richtung $\beta \in$ $H^{1}\left(S_{0}, \mathcal{O}\right)$ gewählt, so definiert diese ein Vektorfeld auf der universellen Jakobischen und damit, via der Lax-Paar-Gleichung $\dot{\mathcal{C}}=\left[\mathcal{C}, \beta(\mathcal{C}, \zeta)^{+}\right], \mathcal{C}=A+\zeta B$, eine Differentialgleichung bzw. ein Vektorfeld auf der Menge der Äquivalenzklassen von Paaren von Matrizen. In unserem Fall ist $F:\left(\mathrm{gl}_{k} \times \mathrm{gl}_{k}\right) / \mathrm{GL}_{k} \rightarrow$ $\mathbb{C}^{\frac{1}{2} k(k+3)}$ definiert durch die Koeffizienten des charakteristischen Polynoms, das Vektorfeld $\mathcal{V}$ definiert durch die Lax-Paar-Bedingung, $M(U)$ die universelle Jakobische und für jeden festen Punkt $c \in \mathbb{C}^{\frac{1}{2} k(k+3)}$ und damit $F^{-1}(c)=M_{S}$ mit einer festen Spektralkurve $S$ und damit fester Jakobischer $J(S)$, der Divisor $D_{c}$ gleich dem Thetadivisor $\Theta \in J(S)$. Wir werden bei der Beschreibung der Balancen einer gegebenen Lax-Paar-Gleichung den folgenden Satz für homogene Vektorfelder benutzen. Homogene Vektorfelder sind Vektorfelder, deren Differentialgleichungen nur aus homogenen Polynomen vom gleichen Grad bestehen. Eine homogene Laurentlösung eines homogenen Vektorfeldes sei von der Form $x_{i}(z)=\frac{1}{z} \sum_{l=0}^{\infty} x_{i}^{(l)} z^{l}$ mit $x^{(0)} \neq 0$.

Satz 3.6. ([AMV04] Theorem 7.25) Sei $V$ ein homogenes polynomiales Vektorfeld auf $\mathbb{C}^{n}$. Ist $x(z)$ eine homogene Balance $z u V$, so ist $x(z)$ für $z \neq 0$ und $|z|$ hinreichend klein konvergent.

\subsection{Painlevé-Analysis}

In diesem Abschnitt untersuchen wir die Menge aller strikten homogenen Laurentlösungen zu einer Lax-Paar-Gleichung $\dot{\mathcal{C}}=\left[\mathcal{C}, \beta(\mathcal{C}, \zeta)^{+}\right]$mit einem 
$\beta \in H^{1}(S, \mathcal{O})$ und $\mathcal{C}=A+\zeta B$. Wir zeigen zunächst, dass für jede Wahl von $\beta$ die Lösung $B$ konstant sein muss. Da wir in Abschnitt 2.5 gezeigt haben, dass jedes Vektorfeld von $J^{g-1}(S)$ den Thetadivisor in allgemeinen Punkten transversal schneidet, können wir, um später Satz 3.1 anzuwenden, ohne Einschränkung eine feste Richtung $\beta=\frac{\eta^{2}}{\zeta} \in H^{1}(S, \mathcal{O})$ wählen. Für deren zugehörige Lax-Paar-Gleichung bestimmen wir dann strikte formale Laurentlösungen. Diese entsprechen dem asymptotischen Verhalten von Äquivalenzklassen von Paaren von Matrizen in Punkten des Thetadivisors, wie der folgende Satz zeigt:

Satz 3.7. Jede strikte homogene Laurentlösung zu $\dot{\mathcal{C}}=\left[\mathcal{C}, \beta(\mathcal{C}, \zeta)^{+}\right]$mit $\mathcal{C}=$ $A+\zeta B$ beschreibt das asymptotische Verhalten von Äquivalenzklassen von Paaren von Matrizen entlang $\beta$, deren Bild in der Jakobischen $J(S)$ einer festen Spektralkurve $S$ gegen einen Punkt des Thetadivisors läuft.

Beweis: Die Lax-Paar-Gleichung $\dot{\mathcal{C}}=\left[\mathcal{C}, \beta(\mathcal{C}, \zeta)^{+}\right]$ist homogen. Nach Satz 3.6 konvergiert jede strikte homogene Laurentlösung $(A(z), B(z))$ für $z \neq 0,|z|$ hinreichend klein. Die $(A(z), B(z))$ definieren jeweils die gleiche Spektralkurve $S=S_{A(z), B(z)}$. Jedes solche $(A(z), B(z))$ definiert damit einen Punkt $L(z)$ in der Jakobischen der Spektralkurve $S$. Da nach Definition der Differentialgleichung $\dot{\mathcal{C}}=\left[\mathcal{C}, \beta(\mathcal{C}, \zeta)^{+}\right]$jeder Fluss $(A(z), B(z)), z \neq 0$, in $\left(\mathrm{gl}_{k} \times \mathrm{gl}_{k}\right) / \mathrm{GL}_{k}$ einem Fluss in $J(S) \backslash \Theta$ zum Vektorfeld definiert durch $\beta$ entspricht und dieser in $J(S)$ eindeutig ist, muss dieser für $z=0$ durch den Thetadivisor laufen (ansonsten gäbe es ein Paar $\left(A_{0}, B_{0}\right) \in\left(\mathrm{gl}_{k} \times \mathrm{gl}_{k}\right) / \mathrm{GL}_{k}$ mit $\lim _{z \rightarrow 0}((A(z), B(z))=$ $\left(A_{0}, B_{0}\right)$, ein Widerspruch zur angenommenen Striktheit).

Satz 3.8. Für jede Laurentlösung $\mathcal{C}(z):=A(z)+\zeta B(z)$ der Lax-Paar-Gleichung $\dot{\mathcal{C}}=[\mathcal{C}, D]$ mit $D=\beta(\mathcal{C}, \zeta)^{+}$und beliebigem $\beta \in H^{1}(S, \mathcal{O})$ ist $B(z)=B$ konstant.

Beweis: Wir stellen $\beta \in H^{1}(S, \mathcal{O})$ wie in Folgerung 2.2 dar als Summe $\sum_{n=2}^{k-1} \eta^{n}\left(\sum_{l=1}^{n-1} a_{n l} \zeta^{l-n}\right)$. Sei $\mathcal{C}(z)=A(z)+\zeta B(z)$ eine Lösung der Lax-PaarGleichung $\mathcal{C}=[\mathcal{C}, D]$. Bezeichne $\left(A^{n-i}: B^{i}\right)$ die Summe aller Wörter bestehend aus $n-i$ mal $A$ und $i$ mal $B$. Z.B. ist $\left(A^{3}: B^{2}\right)=A^{3} B^{2}+A^{2} B A B+$ $A^{2} B^{2} A+A B A^{2} B+A B A B A+A B^{2} A^{2}+B A^{3} B+B A^{2} B A+B A B A^{2}+B^{2} A^{3}$.

Dann ist $A\left(A^{n-i}: B^{i}\right)+B\left(A^{n-i+1}: B^{i-1}\right)=\left(A^{n-i+1}: B^{i}\right)$ sowie 
$\left(A^{n-i}: B^{i}\right) A+\left(A^{n-i+1}: B^{i-1}\right) B=\left(A^{n-i+1}: B^{i}\right)$, und es gilt

$$
\begin{aligned}
D= & \beta(\mathcal{C}, \zeta)^{+}=\left(\sum_{n=2}^{k-1}(A+\zeta B)^{n}\left(\sum_{l=1}^{n-1} a_{n l} \frac{1}{\zeta^{n}} \zeta^{l}\right)\right)^{+} \\
= & \left(\sum_{n=2}^{k-1}\left(\sum_{i=0}^{n}\left(A^{n-i}: B^{i}\right) \zeta^{i}\right)\left(\sum_{l=1}^{n-1} a_{n l} \zeta^{l-n}\right)\right)^{+} \\
= & \zeta^{0} \cdot\left(\sum_{n=2}^{k-1} \sum_{l=1}^{n-1} a_{n l}\left(A^{l}: B^{n-l}\right)\right)+\zeta^{1} \cdot\left(\sum_{n=2}^{k-1} \sum_{l=1}^{n-1} a_{n l}\left(A^{l-1}: B^{n+1-l}\right)\right) \\
& + \text { höhere Potenzen von } \zeta .
\end{aligned}
$$

Damit gilt für den Koeffizienten vor $\zeta$ des Kommutators von $[\mathcal{C}, D]$ :

$$
\begin{aligned}
\dot{B}= & A \cdot\left(\sum_{n=2}^{k-1} \sum_{l=1}^{n-1} a_{n l}\left(A^{l-1}: B^{n+1-l}\right)\right)+B \cdot\left(\sum_{n=2}^{k-1} \sum_{l=1}^{n-1} a_{n l}\left(A^{l}: B^{n-l}\right)\right) \\
& -\left(\sum_{n=2}^{k-1} \sum_{l=1}^{n-1} a_{n l}\left(A^{l-1}: B^{n+1-l}\right)\right) \cdot A-\left(\sum_{n=2}^{k-1} \sum_{l=1}^{n-1} a_{n l}\left(A^{l}: B^{n-l}\right)\right) \cdot B \\
= & \sum_{n=2}^{k-1} \sum_{l=1}^{n-1} a_{n l}\left(A\left(A^{l-1}: B^{n+1-l}\right)+B\left(A^{l}: B^{n-l}\right)\right. \\
= & 0 .
\end{aligned}
$$

Satz 3.9. Allgemein definiert eine Richtung $\beta=\sum_{n=2}^{k-1} \eta^{n}\left(\sum_{l=1}^{n-1} a_{n l} \zeta^{l-n}\right)$ in $H^{1}(S, \mathcal{O})$ auf der Jakobischen via der Lax-Paar-Gleichung die folgende Differentialgleichung auf der Menge der Äquivalenzklassen von Paaren von Matrizen:

$$
\begin{aligned}
& \dot{A}=\sum_{n=2}^{k-1} \sum_{l=1}^{n-1} a_{n l}\left(A\left(A^{l}: B^{n-l}\right)-\left(A^{l}: B^{n-l}\right) A\right) \\
& \dot{B}=0 .
\end{aligned}
$$


Beweis: Wir müssen nur noch (3.2) nachweisen. Allgemein gilt

$$
\begin{aligned}
D & =\beta(C, \zeta)^{+}=\left(\sum_{n=2}^{k-1}(A+\zeta B)^{n}\left(\sum_{l=1}^{n-1} a_{n l} \frac{1}{\zeta^{n}} \zeta^{l}\right)\right)^{+} \\
& =\left(\sum_{n=2}^{k-1}\left(\sum_{i=0}^{n}\left(A^{n-i}: B^{i}\right) \zeta^{i}\right)\left(\sum_{l=1}^{n-1} a_{n l} \zeta^{l-n}\right)\right)^{+} \\
& =\sum_{n=2}^{k-1}\left(\sum_{j=0}^{n-1} \zeta^{j}\left(\sum_{l=j, l \geq 1}^{n-1} a_{n l}\left(A^{l-j}: B^{n+j-l}\right)\right)\right) \\
& =\sum_{j=0}^{k-2} \zeta^{j}\left(\sum_{n=j+1, n \geq 2}^{k-1}\left(\sum_{l=j, l \geq 1}^{n-1} a_{n l}\left(A^{l-j}: B^{n+j-l}\right)\right)\right)
\end{aligned}
$$

und damit

$$
\begin{aligned}
\dot{\mathcal{C}}= & (A+\zeta B)^{\prime} \\
= & {[\mathcal{C}, D] } \\
= & {\left[A+\zeta B, \sum_{j=0}^{k-2} \zeta^{j}\left(\sum_{n=j+1, n \geq 2}^{k-1}\left(\sum_{l=j, l \geq 1}^{n-1} a_{n l}\left(A^{l-j}: B^{n+j-l}\right)\right)\right)\right] } \\
= & \zeta^{0} \sum_{n=2}^{k-1} \sum_{l=1, l \geq 1}^{n-1} a_{n l}\left(A\left(A^{l}: B^{n-l}\right)-\left(A^{l}: B^{n-l}\right) A\right) \\
& +\sum_{j=1}^{k-2} \zeta^{j}\left(\sum_{n=j+1, n \geq 2}^{k-1} \sum_{l=j, l \geq 1}^{n-1} a_{n l}\left(A\left(A^{l-j}: B^{n+j-l}\right)-\left(A^{l-j}: B^{n+j-l}\right) A\right)\right. \\
& \left.+\sum_{n=j, n \geq 2}^{k-1} \sum_{l=j-1, l \geq 1}^{n-1} a_{n l}\left(B\left(A^{l-j+1}: B^{n+j-1-l}\right)-\left(A^{l-j+1}: B^{n+j-1-l}\right) B\right)\right) \\
& +\zeta^{k-1}\left(a_{k-1, k-2}\left(B\left(A^{0}: B^{k-1}\right)-\left(A^{0}: B^{k-1}\right) B\right)\right) \\
& k-1 \\
= & \sum_{n=2}^{n-1} \sum_{l=1}^{n-1} a_{n l}\left(A\left(A^{l}: B^{n-l}\right)-\left(A^{l}: B^{n-l}\right) A\right),
\end{aligned}
$$


da für $j \geq 1$ gilt:

$$
\begin{aligned}
& \sum_{n=j+1, n \geq 2}^{k-1} \sum_{l=j, l \geq 1}^{n-1} a_{n l}\left(\left(A\left(A^{l-j}: B^{n+j-l}\right)-\left(A^{l-j}: B^{n+j-l}\right) A\right)\right) \\
& +\sum_{n=j, n \geq 2}^{k-1} \sum_{l=j-1, l \geq 1}^{n-1} a_{n l}\left(B\left(A^{l-j+1}: B^{n+j-1-l}\right)-\left(A^{l-j+1}: B^{n+j-1-l}\right) B\right) \\
& =\sum_{n=j+1, n \geq 2}^{k-1} \sum_{l=j}^{n-1}\left(a _ { n l } \left(A\left(A^{l-j}: B^{n+j-l}\right)-\left(A^{l-j}: B^{n+j-l}\right) A\right.\right. \\
& =0 .
\end{aligned}
$$

Aufgrund von Satz 3.1 genügt es später, das asymptotische Verhalten von $(A(z), B(z))$ entlang einer fest gewählten Richtung $\beta$ zu betrachten. Wir fixieren ab sofort $\beta=\frac{\eta^{2}}{\zeta}$.

Folgerung 3.10. Für $\beta=\frac{\eta^{2}}{\zeta}$ hat die Differentialgleichung (3.2)-(3.3) die Gestalt

$$
\begin{aligned}
& \dot{A}=\left[A^{2}, B\right] \\
& \dot{B}=0 .
\end{aligned}
$$

Strikte formale homogene Laurentlösungen von (3.4)-(3.5) haben Pole erster Ordnung.

Für formale Laurentlösungen

$$
A=\frac{1}{z} \sum_{l=0}^{\infty} A^{(l)} z^{l}=\sum_{l=0}^{\infty} A^{(l)} z^{l-1}
$$

von (3.4)-(3.5) folgt

$$
\begin{aligned}
\dot{A} & =\sum_{l=0}^{\infty}(l-1) A^{(l)} z^{l-2} \\
& =(A(A: B)-(A: B) A) \\
& =\left[A^{2}, B\right] \\
& =\sum_{l=0}^{\infty}\left(\sum_{q=0}^{l}\left(A^{(q)} A^{(l-q)} B-B A^{(q)} A^{(l-q)}\right)\right) z^{l-2},
\end{aligned}
$$


und damit

$$
\begin{aligned}
-A^{(0)} & =\left[\left(A^{(0)}\right)^{2}, B\right], \\
0 & =\left[A^{(0)} A^{(1)}, B\right]+\left[A^{(1)} A^{(0)}, B\right]
\end{aligned}
$$

und allgemein

$$
(l-1) A^{(l)}=\sum_{q=0}^{l}\left[A^{(q)} A^{(l-q)}, B\right] .
$$

\subsection{Berechnung der indizierenden Menge}

Gesucht sind alle $k \times k$-Matrizen $A^{(0)}$ und $B$, die

$$
-A^{(0)}=\left[\left(A^{(0)}\right)^{2}, B\right]
$$

erfüllen. Die Lösungsmenge wird indizierende Menge genannt.

Offensichtlich sind die Lösungen invariant unter Konjugation, d.h. wir können ohne Einschränkung annehmen, dass $A^{(0)}$ in Jordanscher Normalform vorliegt. Gesucht sind also $a_{i j}:=a_{i j}^{(0)} \in \mathbb{C}, 1 \leq i, j \leq k$ mit

$$
\begin{aligned}
a_{i j}= & \sum_{c=1}^{k} \sum_{d=1}^{k}\left(-a_{i d} a_{d c} b_{c j}+b_{i d} a_{d c} a_{c j}\right) \\
= & a_{j j}^{2} b_{i j}+a_{j j} a_{j-1, j} b_{i, j-1}+a_{j-1 j} a_{j-1, j-1} b_{i, j-1}+a_{j-1, j} a_{j-2, j-1} b_{i, j-2} \\
& -a_{i i}^{2} b_{i j}-a_{i i} a_{i, i+1} b_{i+1, j}-a_{i, i+1} a_{i+1, i+1} b_{i+1, j}-a_{i, i+1} a_{i+1, i+2} b_{i+2, j} .
\end{aligned}
$$

Allgemein gilt

Satz 3.11. Liegt $A^{(0)}$ in Jordanscher Normalform vor, so sind alle Diagonaleinträge von $A^{(0)}$ gleich null.

Beweis: Mit (3.10) erhalten wir sofort, dass, falls $a_{l-1, l}=a_{l, l+1}=0$ gilt, auch $a_{l l}=0$ folgt. Insbesondere gilt $A^{(0)}=0$, falls $A^{(0)}$ Diagonalgestalt hat. In diesem Fall liegt keine strikte formale Laurentreihe vor.

Wir betrachten nun Jordanblöcke mit $J>0$ Einsen, sei also $a_{l, l+1}=1$ für $l=n, \ldots, n+J-1$ für ein $1 \leq n \leq k-J$ und $a_{n-1, n}=a_{n+J, n+J+1}=0$. Der Fall $J=1$ ist trivial, da $a_{n, n}=a=-2 a b_{n+1, n}$ und $a_{n+1, n+1}=a=2 a b_{n+1, n}$ gilt. Sei also $J>1$. Dann ist $a:=a_{n n}=\ldots=a_{n+J, n+J}$, und wir erhalten mit 
$(3.10)$

$$
\begin{aligned}
a_{n, n}= & a=-2 a b_{n+1, n}-b_{n+2, n} \\
a_{n+1, n+1}= & a=2 a b_{n+1, n}-2 a b_{n+2, n+1}-b_{n+3, n+1} \\
\vdots & \\
a_{n+i, n+i}=a=2 a b_{n+i, n+i-1}+b_{n+i, n+i-2}-2 a b_{n+i+1, n+i} & -b_{n+i+2, n+i}, \quad 2 \leq i \leq J-2, \\
\vdots & \\
a_{n+J-1, n+J-1}=a & =2 a b_{n+J-1, n+J-2}+b_{n+J-1, n+J-3}-2 a b_{n+J, n+J-1} \\
a_{n+J, n+J}= & a=2 a b_{n+J, n+J-1}+b_{n+J, n+J-2},
\end{aligned}
$$

und damit

$$
\begin{aligned}
(J+1) a=- & 2 a b_{n+1, n}-b_{n+2, n}+2 a b_{n+1, n}-2 a b_{n+2, n+1}-b_{n+3, n+1} \\
& +\sum_{i=2}^{J-2}\left(2 a b_{n+i, n+i-1}+b_{n+i, n+i-2}-2 a b_{n+i+1, n+i}-b_{n+i+2, n+i}\right) \\
& +2 a b_{n+J-1, n+J-2}+b_{n+J-1, n+J-3}-2 a b_{n+J, n+J-1} \\
& +2 a b_{n+J, n+J-1}+b_{n+J, n+J-2} \\
=0, &
\end{aligned}
$$

folglich

$$
a=0
$$

Wir zeigen nun, dass nur Jordanblöcke der Länge $2 l+1$, d.h. mit gerade vielen, $J=2 l$, Einsen, vorkommen können, $l \in \mathbb{N}$.

Satz 3.12. Liegt $A^{(0)}$ in Jordanscher Normalform vor, so besteht $A^{(0)}$ nur aus Jordanblöcken ungerader Länge, d.h. mit gerade vielen Einsen.

Beweis: Da nach Satz 3.11 alle Diagonaleinträge von $A^{(0)}$ null sind, gilt

$$
a_{i j}=a_{j-1, j} a_{j-2, j-1} b_{i, j-2}-a_{i, i+1} a_{i+1, i+2} b_{i+2, j}
$$

für $1 \leq i, j \leq k, a_{01}=a_{-10}=a_{k, k+1}=a_{k+1, k+2}=0$, und speziell für ein $n$ mit $1 \leq n<k$

$$
a_{n, n+1}=a_{n, n+1}\left(a_{n-1, n} b_{n, n-1}-a_{n+1, n+2} b_{n+2, n+1}\right) .
$$

Angenommen $A^{(0)}$ besitzt einen Jordanblock mit ungerade vielen, d.h. mit $0<J=2 l+1$ Einsen für eine natürliche Zahl $l \in \mathbb{N}$. 
Es gelte also $a_{n-1, n}=a_{n+J, n+J+1}=0$ für ein $1 \leq n \leq k-J$ und $a_{n+i, n+i+1}=1$ für $i=0, \ldots, J-1$. Insbesondere folgt aus (3.11) sofort $J>1$. Dann gilt

$$
\begin{aligned}
a_{n, n+1} & =1=-b_{n+2, n+1} \\
a_{n+1, n+2} & =1=b_{n+1, n}-b_{n+3, n+2} \\
\vdots & \\
a_{n+i, n+i+1} & =1=b_{n+i, n+i-1}-b_{n+i+2, n+i+1}, \quad 1 \leq i \leq J-2=2 l-1, \\
\vdots & \\
a_{n+2 l, n+2 l+1} & =1=b_{n+2 l, n+2 l-1},
\end{aligned}
$$

und damit

$$
\begin{aligned}
\sum_{i=0}^{l} a_{n+2 i, n+2 i+1} & =l+1 \\
& =\frac{J+1}{2} \\
& =-b_{n+2, n+1}+\sum_{i=1}^{l-1}\left(b_{n+2 i, n+2 i-1}-b_{n+2 i+2, n+2 i+1}\right)+b_{n+2 l, n+2 l-1} \\
& =0,
\end{aligned}
$$

ein Widerspruch zu $J>1$.

Lösungen von $(3.9)$ sind somit von der Form $\left(A^{(0)}, B\right)$ mit $A^{(0)}$ bestehend aus $\ell$ Jordanblöcken der Länge $2 l_{1}+1, \ldots, 2 l_{\ell}+1$ mit jeweils $2 l_{1}, \ldots, 2 l_{\ell}$ Einsen, $l_{i} \in \mathbb{N}$, d.h. mit

$$
\begin{gathered}
a_{n_{1}, n_{1}+1}=\ldots=a_{n_{1}+2 l_{1}-1, n_{1}+2 l_{1}}=1, a_{n_{1}+2 l_{1}, n_{1}+2 l_{1}+1}=0 \\
a_{n_{2}, n_{2}+1}=\ldots=a_{n_{2}+2 l_{2}-1, n_{2}+2 l_{2}}=1, a_{n_{2}+2 l_{2}, n_{2}+2 l_{2}+1}=a_{n_{2}-1, n_{2}}=0 \\
\quad \vdots \\
a_{n_{\ell}, n_{\ell}+1}=\ldots=a_{n_{\ell}+2 l_{\ell}-1, n_{\ell}+2 l_{\ell}}=1, a_{n_{\ell}+2 l_{\ell}, n_{\ell}+2 l_{\ell}+1}=a_{n_{\ell}-1, n_{\ell}}=0,
\end{gathered}
$$

wobei wir ohne Einschränkung $1=n_{1}<n_{2}<\ldots<n_{\ell}<k-1$ annehmen.

Für die konstante Matrix $B$ erhalten wir aus

$$
a_{i j}=a_{j-1, j} a_{j-2, j-1} b_{i, j-2}-a_{i, i+1} a_{i+1, i+2} b_{i+2, j}
$$

die folgenden Bedingungen: 
1. Für $i \in\left\{n_{c}, \ldots, n_{c}+2 l_{c}-2\right\}$ für ein $1 \leq c \leq \ell$ und $j \notin\left\{n_{d}+2, \ldots, n_{d}+2 l_{d}\right\}$ für alle $d \in\{1, \ldots, \ell\}$ ist

$$
a_{j-1, j} a_{j-2, j-1}=0 \text { und } a_{i, i+1} a_{i+1, i+2}=1 .
$$

Wir erhalten

- $0=-b_{i+2, j}$ falls $j \neq i+1$ und

- $1=-b_{i+2, j}$ falls $j=i+1$, und damit $i=n_{c}$.

2. Für $j \in\left\{n_{c}+2, \ldots, n_{c}+2 l_{c}\right\}$ für ein $1 \leq c \leq \ell$ und $i \notin\left\{n_{d}, \ldots, n_{c}+2 l_{d}-2\right\}$ für alle $d \in\{1, \ldots, \ell\}$ ist

$$
a_{j-1, j} a_{j-2, j-1}=1 \text { und } a_{i, i+1} a_{i+1, i+2}=0 .
$$

Es folgt

- $0=b_{i, j-2}$ falls $j \neq i+1$

- $1=b_{i, j-2}$ falls $j=i+1$ und damit $i=n_{c}+2 l_{c}-1$.

3. Für $i \in\left\{n_{c}, \ldots, n_{c}+2 l_{c}-2\right\}$ für ein $1 \leq c \leq \ell$ und $j \in\left\{n_{d}+2, \ldots, n_{d}+2 l_{d}\right\}$ für ein $1 \leq d \leq \ell$ gilt

$$
a_{j-1, j} a_{j-2, j-1}=1=a_{i, i+1} a_{i+1, i+2} .
$$

Es folgt

- $b_{i, j-2}=b_{i+2, j}$ falls $j \neq i+1$

- $1=b_{i, j-2}-b_{i+2, j}$ falls $j=i+1$.

Da für alle anderen Kombinationen von $i$ und $j$ die $a_{i j}$ verschwinden, sind alle anderen Einträge von $B$ unbestimmt.

Beispiel 3.13. 1. Besteht $A^{(0)}$ nur aus einem Jordanblock mit 2 Einsen, so sind $A^{(0)}$ und $B$ von der Form

$$
A^{(0)}=\left(\begin{array}{cccccc}
0 & 1 & 0 & 0 & \cdots & 0 \\
0 & 0 & 1 & 0 & \cdots & 0 \\
0 & \cdots & & & & 0 \\
\vdots & & & & & \vdots \\
\vdots & & & & & \vdots \\
0 & \cdots & & & & 0
\end{array}\right), B=\left(\begin{array}{cccccc}
b_{11} & & & & & \\
1 & b_{22} & & & * & \\
0 & -1 & b_{11} & 0 & \cdots & 0 \\
0 & & & & & \\
\vdots & & * & & & \\
0 & & & & & b_{k k}
\end{array}\right) .
$$

Wir werden später sehen, dass hierdurch eine Hauptbalance definiert wird. 
2. Besteht $A^{(0)}$ nur aus einem Jordanblock mit 4 Einsen, so gilt

$$
A^{(0)}=\left(\begin{array}{cccccccc}
0 & 1 & 0 & 0 & 0 & 0 & \cdots & 0 \\
0 & 0 & 1 & 0 & 0 & 0 & \cdots & 0 \\
0 & 0 & 0 & 1 & 0 & & \cdots & 0 \\
0 & 0 & 0 & 0 & 1 & 0 & \vdots & 0 \\
0 & \cdots & & & & & & 0 \\
\vdots & & & & & & & \vdots \\
\vdots & & & & & & & \vdots \\
0 & \cdots & & & & & & 0
\end{array}\right)
$$

und

$$
B=\left(\begin{array}{cccccccc}
b_{11} & b_{12} & b_{13} & & & & & \\
2 & b_{22} & b_{23} & & & * & & \\
0 & -1 & b_{11} & b_{12} & b_{13} & 0 & \cdots & 0 \\
0 & 0 & 1 & b_{22} & b_{23} & 0 & \cdots & 0 \\
0 & 0 & 0 & -2 & b_{11} & 0 & \cdots & 0 \\
\vdots & \vdots & \vdots & & & & & \\
\vdots & \vdots & \vdots & & * & & & \\
0 & 0 & 0 & & & & & b_{k k}
\end{array}\right)
$$

3. Besteht $A^{(0)}$ aus einem Jordanblock mit $k-1$ Einsen, so gilt

$$
B=\left(\begin{array}{ccccccc}
b_{11} & b_{12} & b_{13} & b_{14} & & \cdots & b_{1 k} \\
\frac{k-1}{2} & b_{22} & b_{23} & b_{24} & & \cdots & b_{2 k} \\
0 & -1 & b_{11} & b_{12} & & \cdots & b_{1, k-2} \\
0 & 0 & \frac{k-3}{2} & b_{22} & & \cdots & b_{2, k-2} \\
0 & 0 & 0 & -2 & b_{11} & \cdots & b_{1, k-4} \\
\vdots & & & & & & \\
0 & 0 & 0 & \cdots & 0 & -\frac{k-1}{2} & b_{11}
\end{array}\right)
$$

Hierbei ist die Menge aller Äquivalenzklassen von Lösungen von (3.9) durch feste $A^{(0)}$ und die Menge aller zugehörigen oben definierten $B=B\left(A^{(0)}\right)$ modulo Konjugation mit Matrizen $X$ aus dem Stabilisator von $A^{(0)}$ gegeben. Zum Beispiel besitzen Matrizen $X$ des Stabilisators von $A^{(0)}$ wie in (3.13) 
insgesamt $k^{2}-4 k+5$ freie Parameter und sind von der Form

$$
X=\left(\begin{array}{cccccc}
x_{11} & x_{12} & x_{13} & & \ldots & x_{1 k} \\
0 & x_{11} & x_{12} & 0 & \ldots & 0 \\
\vdots & 0 & x_{11} & 0 & \ldots & 0 \\
\vdots & \vdots & & & & \\
& & & * & & \\
0 & 0 & & & &
\end{array}\right)
$$

d.h. $B$ modulo $X$ besteht aus $(k-1)(k-3)+2 k-1-\left(k^{2}-4 k+5\right)=2 k-3$ freien Parametern.

\subsection{Die Kowalevski-Matrix}

Alle weiteren Koeffizienten $v\left(a^{(l)}\right):=\left(a_{11}^{(l)}, a_{12}^{(l)}, \ldots, a_{k k}^{(l)}\right)^{t}$ mit $l \geq 1$ von formalen strikten Laurentlösungen lassen sich induktiv aus der indizierenden Menge durch Lösen des linearen Gleichungssystems $K(l) v\left(a^{(l)}\right)=v\left(a^{(0)}, \ldots, a^{(l-1)}\right)$ mit einer $k^{2} \times k^{2}$-Matrix $K(l)$, der sogenannten Kowalevski-Matrix, berechnen. Die Lösungen sind nicht eindeutig. Die Anzahl der insgesamt frei wählbaren Parameter gibt an, um welche Art von Balance es sich bei der Lösungsmenge handelt.

\section{Formale Laurentlösungen komponentenweise}

Betrachten wir komponentenweise formale Laurentreihen

$$
a_{i j}=\sum_{l=0}^{\infty} a_{i j}^{(l)} z^{l-1},
$$

so folgt aus (3.8) die Bedingung

$$
\begin{aligned}
0 & =(l-1) a_{i j}^{(l)}-\sum_{q=0}^{l} \sum_{n=1}^{k}\left(\left(A^{(q)} A^{(l-q)}\right)_{i n} b_{n j}-b_{i n}\left(A^{(q)} A^{(l-q)}\right)_{n j}\right) \\
& =(l-1) a_{i j}^{(l)}-\sum_{q=0}^{l} \sum_{n=1}^{k} \sum_{m=1}^{k}\left(a_{i m}^{(q)} a_{m n}^{(l-q)} b_{n j}-b_{i n} a_{n m}^{(q)} a_{m j}^{(l-q)}\right)
\end{aligned}
$$

an die $a_{i j}^{(l)}$. Die indizierende Menge ist durch Lösungen der Gleichung

$$
a_{i j}^{(0)}+\sum_{n=1}^{k} \sum_{m=1}^{k}\left(a_{i m}^{(0)} a_{m n}^{(0)} b_{n j}-a_{n m}^{(0)} a_{m j}^{(0)} b_{i n}\right)=0
$$


gegeben. Die Koeffizienten $a_{i j}^{(l)}$ der formalen Laurentlösungen lassen sich für $l \geq 1$ aus ihnen mittels

$$
K(l)\left(\begin{array}{c}
a_{11}^{(l)} \\
a_{12}^{(l)} \\
\vdots \\
a_{i j}^{(l)} \\
\vdots \\
a_{k k}^{(l)}
\end{array}\right)=\sum_{q=1}^{l-1} \sum_{n=1}^{k} \sum_{m=1}^{k}\left(\begin{array}{c}
a_{1 m}^{(q)} a_{m n}^{(l-q)} b_{n 1}-a_{n m}^{(q)} a_{m 1}^{(l-q)} b_{1 n} \\
a_{1 m}^{(q)} a_{m n}^{(l-q)} b_{n 2}-a_{n m}^{(q)} a_{m 2}^{(l-q)} b_{1 n} \\
\vdots \\
a_{i m}^{(q)} a_{m n}^{(l-q)} b_{n j}-b_{i n} a_{n m}^{(q)} a_{m j}^{(l-q)} \\
\vdots \\
a_{k m}^{(q)} a_{m n}^{(l-q)} b_{n k}-a_{n m}^{(q)} a_{m k}^{(l-q)} b_{k n}
\end{array}\right)
$$

berechnen, wobei die Einträge $(K(l))_{(i j),(c d)}$ der $k^{2} \times k^{2}$-Matrix $K(l)$ gegeben sind durch

$$
\begin{cases}(l-1)-\sum_{n=1}^{k} a_{j n}^{(0)} b_{n j}-a_{i i}^{(0)} b_{j j}+\sum_{n=1}^{k} b_{i n} a_{n i}^{(0)}+b_{i i} a_{j j}^{(0)} & \text { falls }(i j)=(c d) \\ -\sum_{n=1}^{k} a_{d n}^{(0)} b_{n j}-a_{i i}^{(0)} b_{d j}+b_{i i} a_{d j}^{(0)} & \text { falls } i=c, j \neq d \\ -a_{i c}^{(0)} b_{j j}+\sum_{n=1}^{k} b_{i n} a_{n c}^{(0)}+b_{i c} a_{j j}^{(0)} & \text { falls } i \neq c, j=d \\ -a_{i c}^{(0)} b_{d j}+b_{i c} a_{d j}^{(0)} & \text { falls } i \neq c, j \neq d\end{cases}
$$

Definition 3.14. $K(l)=: K(l)(k)$ ist die sogenannte Kowalevski-Matrix.

Die Nullstellen der Determinante der Kowalevski-Matrix geben an, für welche $l$ die Matrizen $A^{(l)}$ freie Parameter besitzen. Für alle anderen $l$ ist $K(l)$ invertierbar, d.h. $A^{(l)}$ eindeutig durch alle $A^{(j)}$ mit $j<l$ bestimmt.

Bemerkung 3.15. Die Determinante der $k^{2} \times k^{2}$-Kowalewski-Matrix (3.15) $z u$ (3.13), d.h. zu $A^{(0)}$ bestehend aus nur einem Jordanblock mit zwei Einsen und zugehörigem B, ist das Produkt ihrer Diagonaleinträge, und es gilt

$$
\operatorname{det} K(l)=(l+1) l^{2 k-4}(l-1)^{k^{2}-4 k+6}(l-2)^{2 k-4}(l-3) .
$$

Beweis: (Induktion nach $k$ ):

- Induktionsanfang: Sei $k=3$. Dann hat $K(l)(3)$ die Gestalt

$$
\left(\begin{array}{ccccccccc}
l-2 & 0 & 0 & 0 & -1 & 0 & b_{12} & 0 & 0 \\
b_{11}-b_{22} & l & 0 & 0 & b_{11}-b_{22} & 1 & b_{13} & b_{12} & 0 \\
-b_{23} & 0 & l-1 & -b_{13} & b_{12}-b_{23} & 0 & 0 & b_{13} & b_{12} \\
0 & 0 & 0 & l-1 & 0 & 0 & b_{22}-b_{11} & -1 & 0 \\
1 & 0 & 0 & 0 & l+1 & 0 & b_{23}-b_{12} & 0 & 1 \\
0 & 1 & 0 & -b_{23} & b_{22}-b_{11} & l & -b_{13} & 0 & b_{22}-b_{11} \\
0 & 0 & 0 & 0 & 0 & 0 & l-3 & 0 & 0 \\
0 & 0 & 0 & -1 & 0 & 0 & b_{11}-b_{22} & l-1 & 0 \\
0 & 0 & 0 & 0 & -1 & 0 & -b_{23} & 0 & l-2
\end{array}\right)
$$


Ihre Determinante ist $(l+1) l^{2}(l-1)^{3}(l-2)^{2}(l-3)$.

- Induktionsschritt $(k-1 \rightarrow k)$ :

$K(l)(k)$ entsteht aus $K(l)(k-1)$ durch Hinzufügen der Zeilen $Z(1, k)$ bis $Z(k-1, k)$, der Spalten $S(1, k)$ bis $S(k-1, k)$ und der $k+k-1=$ $2 k-1$ quadratischen $k \times k$-Matrizen $M(k, c), M(i, k)$ mit $1 \leq i, c \leq$ $k$ gegeben durch $(M(k, c))_{j, d}=(K(l)(k))_{(k, j),(c, d)}$ bzw. $(M(i, k))_{j, d}=$ $(K(l)(k))_{(i, j),(k, d)}$.

Insbesondere ist $M(k, k)$ eine untere Dreiecksmatrix, da $(M(k, k))_{j, d}=$ $(K(l)(k))_{(k, j),(k, d)}$ für $d>j$ gleich null ist. Dies liegt daran, dass nach Voraussetzung $A^{(0)}$ wie in (3.13) nur aus einem Jordanblock mit zwei Einsen besteht und $b_{31}=0$ gilt. Damit ist die Determinante von $M(k, k)$ gleich dem Produkt ihrer Diagonaleinträge. Da jetzt $k>3$ gilt und damit für $i=1, \ldots, k-1$ jeweils die letzte Spalte von $M(i, k)$ gleich null ist $\left((M(i, k))_{j, k}=(K(l)(k))_{(i, j),(k, k)}=0\right)$, ist $\operatorname{det}(M(i, k))=0$ für $i=1, \ldots, k-1$. Außerdem sind in jeder Spalte $(i, k)$ sowohl die Einträge $K_{(i, j),(i, k)}(l)(k)$ für $j=1, \ldots, k-1$ über dem Diagonaleintrag, also auch die Einträge $K_{(i+1, j)(i, k)}(l)(k)$ für $j=1, \ldots, k-1$ unter dem Diagonaleintrag gleich null.

Tauschen wir also die Zeilen $Z(1, k)$ bis $Z(k-2, k)$ zu einem Block über der Zeile $(k-1, k)$ nach unten und die Spalten $(1, k)$ bis $(k-2, k) \mathrm{zu}$ einem Block neben der Spalte $(k-1, k)$ nach rechts, so verändert dies nicht die Determinante von $K(l)(k)$, und die neue Matrix $\widetilde{K(l)(k)}$ hat die Gestalt 


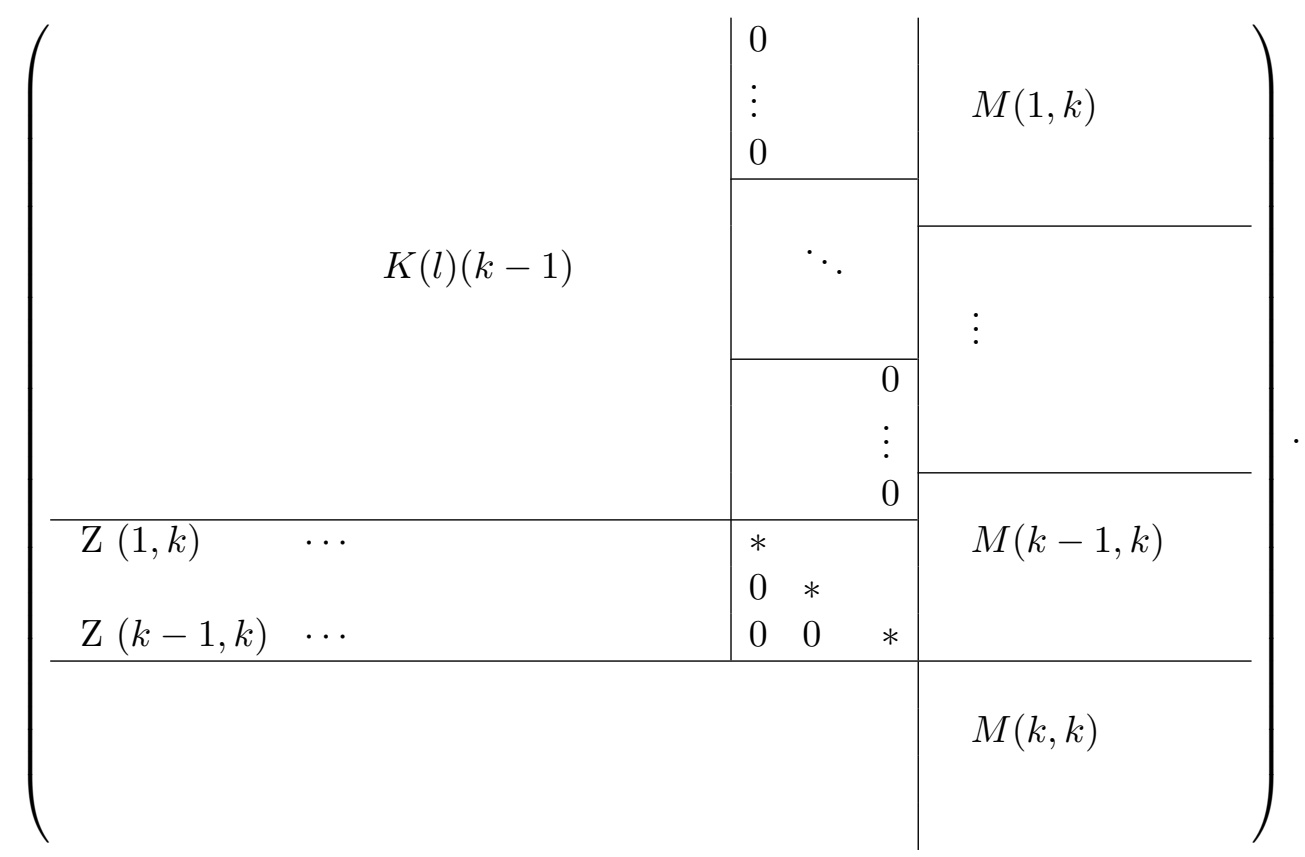

Damit folgt nach Induktionsvoraussetzung, dass die Determinante von $K(l)(k)$ gleich dem Produkt der Diagonaleinträge ist. Mit

$$
(K(l))_{(i, j)(i, j)}=l-1-\left\{\begin{array}{ll}
1 & \text { falls } j=1 \\
-1 & \text { falls } j=2 \\
0 & \text { sonst }
\end{array}+ \begin{cases}1 & \text { falls } i=2 \\
-1 & \text { falls } i=3 \\
0 & \text { sonst }\end{cases}\right.
$$

erhalten wir

$$
\operatorname{det} K(l)=(l+1) l^{2 k-4}(l-1)^{k^{2}-4 k+6}(l-2)^{2 k-4}(l-3) .
$$

\subsection{Bestimmung weiterer Koeffizienten}

Wir haben in Abschnitt 3.3 gesehen, dass für $A^{(0)}$ wie in (3.13), d.h. mit $A^{(0)}$ bestehend aus nur einem Jordanblock mit zwei Einsen, die Matrix $B$ von $2 k-3$ Parametern abhängt. Können wir zeigen, dass die zugehörigen Koeffizienten $A^{(1)}$ von $k^{2}-4 k+6$ Parametern, $A^{(2)}$ von $2 k-4$ Parametern und $A^{(3)}$ von einem Parameter abhängen, so hängt die zugehörige Balance von $2 k-3+k^{2}-4 k+6+2 k-4+1=k^{2}$ Parametern ab, ist also mit 
Satz 3.7 eine Hauptbalance (da $\operatorname{dim}\left(\mathrm{gl}_{k} \times \mathrm{gl}_{k}\right) / \mathrm{GL}_{k}=k^{2}+1$ gilt). Für $A^{(3)}$ ist die Behauptung klar, da $(l-3)$ nur mit Vielfachheit eins ein Faktor der Determinante von $K(l)$ ist. Im folgenden Abschnitt beschäftigen wir uns mit den Koeffizienten $A^{(1)}$ und $A^{(2)}$.

\section{Bestimmung von $A^{(1)}$}

Wir bestimmen zur Balance (3.13) den Koeffizienten $A^{(1)}$ der formalen Laurentreihe $A=\sum_{l=0}^{\infty} A^{(l)} z^{l-1}$. Wir erinnern daran, dass dieser nach (3.7) definiert ist durch $0=\left[A^{(0)} A^{(1)}+A^{(1)} A^{(0)}, B\right]$. Mit

$$
\left(A^{(0)} A^{(1)}\right)_{i j}= \begin{cases}0 & \text { falls } i \geq 3 \\ a_{i+1, j}^{(1)} & \text { falls } i=1,2\end{cases}
$$

und

$$
\left(A^{(1)} A^{(0)}\right)_{i j}= \begin{cases}0 & \text { falls } j=1 \text { oder } j \geq 4 \\ a_{i, j-1}^{(1)} & \text { falls } j=2,3\end{cases}
$$

erhalten wir

$$
\begin{aligned}
0 & =\left(\left[A^{(0)} A^{(1)}+A^{(1)} A^{(0)}, B\right]\right)_{i j} \\
& =\sum_{l=1}^{k}\left(\left(A^{(0)} A^{(1)}\right)_{i l} b_{l j}+\left(A^{(1)} A^{(0)}\right)_{i l} b_{l j}-b_{i l}\left(A^{(0)} A^{(1)}\right)_{l j}-b_{i l}\left(A^{(1)} A^{(0)}\right)_{l j}\right) .
\end{aligned}
$$

Damit folgt

$$
\begin{aligned}
& 0=\left(\left[A^{(0)} A^{(1)}+A^{(1)} A^{(0)}, B\right]\right)_{31}=2 a_{31}^{(1)}=0 \\
& 0=\left(\left[A^{(0)} A^{(1)}+A^{(1)} A^{(0)}, B\right]\right)_{i 1}=a_{i 1}^{(1)}=0 \quad \text { für } i>3 \\
& 0=\left(\left[A^{(0)} A^{(1)}+A^{(1)} A^{(0)}, B\right]\right)_{3 j}=a_{3 j}^{(1)}=0 \quad \text { für } j \geq 4 \\
& 0=\left(\left[A^{(0)} A^{(1)}+A^{(1)} A^{(0)}, B\right]\right)_{32}=-a_{21}^{(1)}=0 \\
& 0=\left(\left[A^{(0)} A^{(1)}+A^{(1)} A^{(0)}, B\right]\right)_{11}=a_{22}^{(1)}+a_{11}^{(1)}=0 \\
& 0=\left(\left[A^{(0)} A^{(1)}+A^{(1)} A^{(0)}, B\right]\right)_{21}=a_{32}^{(1)}=0 \\
& 0=\left(\left[A^{(0)} A^{(1)}+A^{(1)} A^{(0)}, B\right]\right)_{33}=a_{33}^{(1)}+a_{22}^{(1)}=0 \\
& 0=\left(\left[A^{(0)} A^{(1)}+A^{(1)} A^{(0)}, B\right]\right)_{2 j}=-a_{2 j}^{(1)}=0 \quad \text { für } j \geq 4 \\
& 0=\left(\left[A^{(0)} A^{(1)}+A^{(1)} A^{(0)}, B\right]\right)_{i 2}=-a_{i 2}^{(1)}=0 \quad \text { für } i \geq 3 \\
& 0=\left(\left[A^{(0)} A^{(1)}+A^{(1)} A^{(0)}, B\right]\right)_{23}=-a_{23}^{(1)}-a_{12}^{(1)}=0 .
\end{aligned}
$$


Somit ist die Matrix $A^{(1)}$ von der Form

$$
A^{(1)}=\left(\begin{array}{cccccc}
a_{11}^{(1)} & a_{12}^{(1)} & a_{13}^{(1)} & \cdots & & a_{1 k}^{(1)} \\
0 & -a_{11}^{(1)} & -a_{12}^{(1)} & 0 & \cdots & 0 \\
0 & 0 & a_{11}^{(1)} & 0 & \cdots & 0 \\
0 & 0 & a_{43}^{(1)} & a_{44}^{(1)} & \cdots & a_{4 k}^{(1)} \\
\vdots & \vdots & \vdots & & & \vdots \\
0 & 0 & a_{k 3}^{(1)} & \cdots & & a_{k k}^{(1)}
\end{array}\right)
$$

ohne weitere Bedingungen an die verbliebenen $a_{i j}^{(1)}$, da alle Einträge $\left(\left[A^{(0)} A^{(1)}+\right.\right.$ $\left.\left.A^{(1)} A^{(0)}, B\right]\right)_{i j}$ mit dieser Wahl bereits verschwinden. Insbesondere hängt $A^{(1)}$ von $k^{2}-4 k+6$ freien Parametern ab.

\section{Bestimmung von $A^{(2)}$}

Der Koeffizient $A^{(2)}$ ist schwerer zu bestimmen, da er von $B, A^{(0)}$ und $A^{(1)}$ abhängt. (Man beachte hierbei, dass wir zwar in Beispiel 3.13 gezeigt haben, dass $B$ nur von $2 k-3$ Parametern abhängt, wir aber weiterhin die Darstellung (3.13) wie in Beispiel 3.13 für $B$ verwenden, da eine Normalform von $B$ keine Vereinfachung der Bedingungen an $A^{(2)}$ bewirkt.) Wir zeigen im Folgenden nur den

Satz 3.16. $A^{(2)}$ hängt von $2 k-4$ freien Parametern $a b$.

Beweis: Wegen (3.8) für $l=2$ erfüllt $A^{(2)}$ die Bedingung

$$
A^{(2)}=\left[A^{(0)} A^{(2)}, B\right]+\left[A^{(1)^{2}}, B\right]+\left[A^{(2)} A^{(0)}, B\right],
$$

bzw. in Koordinaten (siehe (3.14) für $l=2$ )

$$
K(2)\left(\begin{array}{c}
a_{11}^{(2)} \\
a_{12}^{(2)} \\
\vdots \\
a_{i j}^{(2)} \\
\vdots \\
a_{k k}^{(2)}
\end{array}\right)=\underbrace{\sum_{n=1}^{k} \sum_{m=1}^{k}\left(\begin{array}{c}
a_{1 m}^{(1)} a_{m n}^{(1)} b_{n 1}-a_{n m}^{(1)} a_{m 1}^{(1)} b_{1 n} \\
a_{1 m}^{(1)} a_{m n}^{(1)} b_{n 2}-a_{n m}^{(1)} a_{m 2}^{(1)} b_{1 n} \\
\vdots \\
a_{i m}^{(1)} a_{m n}^{(1)} b_{n j}-b_{i n} a_{n m}^{(1)} a_{m j}^{(1)} \\
\vdots \\
a_{k m}^{(1)} a_{m n}^{(1)} b_{n k}-a_{n m}^{(1)} a_{m k}^{(1)} b_{k n}
\end{array}\right)}_{=: \vec{w}} .
$$

Damit folgt für $A^{(2)}$ sofort $a_{31}^{(2)}=0, a_{22}^{(2)}=0$ und $a_{21}^{(2)}=a_{32}^{(2)}$. Wir zeigen nun, dass der Rang rang $(K(2))$ gleich dem Rang der erweiterten Matrix $\operatorname{rang}(K(2): \vec{w})$ gleich $k^{2}-(2 k-4)$ ist, und damit die Behauptung. 
Da $a_{31}^{(2)}=a_{22}^{(2)}=0$ gilt, zeigen wir stattdessen $\operatorname{rang}(\widetilde{K(l)})=\operatorname{rang}(\widetilde{K(2)}: \vec{w})=$ $k^{2}-2 k+2$, wobei die $k^{2} \times\left(k^{2}-2\right)$-Matrix $\widetilde{K(2)}$ durch Streichen der Spalten $(3,1)$ und $(2,2)$ von $K(2)$ entsteht. Man rechnet leicht nach, dass dann alle Einträge der insgesamt $2 k-3$ Zeilen $(3,1),(1,1)$ sowie $(3, j)$ und $(i, 1)$ von $\widehat{K(2)}$ für $i>3, j>2$ gleich null sind, genauso wie die entsprechenen Einträge des Vektors $\vec{w}$. Außerdem ist die Zeile $(2,1)$ gleich der Zeile $(3,2)$. Damit ist der Rang von $\widetilde{K(2)}$ höchstens gleich $k^{2}-2 k+2$. Betrachtet man nun die Spalten $(1, j), j \geq 2,(2,1),(1,1),(2, j), j \geq 3$, und $(i, j)$ mit $i>3, j \neq 1$, so kann man hier einen Gaußschen Algorithmus durchführen und erhält die lineare Unabhängigkeit dieser Vektoren, d.h. $\operatorname{rang}(\widetilde{K(2)})=\operatorname{rang}(\widetilde{K(2)}: w)=k^{2}-2 k+2$ und damit $\operatorname{rang}(K(2))=k^{2}-2 k+4$, d.h. die Menge der Lösungen $A^{(2)}$ hängt von $2 k-4$ Parametern ab.

Beispiel 3.17. Für $k=3$ ist die Matrix $A^{(2)}$ von der Form

$$
\left(\begin{array}{ccc}
-a_{33}^{(2)} & \frac{\left(b_{11}-b_{22}\right) a_{33}^{(2)}-\left(2 b_{12}+b_{23}\right) a_{32}^{(2)}+c}{3} & -a_{33}^{(2)}\left(b_{12}+b_{23}\right) \\
a_{32}^{(2)} & 0 & \frac{\left.\left(b_{11}-b_{22}\right) a_{33}^{(2)}+\left(2 b_{23}+b_{12}\right) a_{32}^{(2)}+c\right)}{3} \\
0 & a_{32}^{(2)} & a_{33}^{(2)}
\end{array}\right)
$$

mit $c:=-2 a_{11}^{(1)} a_{13}^{(1)}+\left(a_{12}^{(1)}\right)^{2}$.

\section{Ergebnis}

Insgesamt erhalten wir den folgenden

Satz 3.18. Ist

$$
A^{(0)}=\left(\begin{array}{cccccc}
0 & 1 & 0 & 0 & \cdots & 0 \\
0 & 0 & 1 & 0 & \cdots & 0 \\
0 & \cdots & & & & 0 \\
\vdots & & & & & \vdots \\
\vdots & & & & & \vdots \\
0 & \cdots & & & & 0
\end{array}\right), B=\left(\begin{array}{ccccccc}
b_{11} & & & & & \\
1 & b_{22} & & & * & \\
0 & -1 & b_{11} & 0 & \cdots & 0 \\
0 & & & & & \\
\vdots & & * & & & \\
0 & & & & & b_{k k}
\end{array}\right)
$$

eine Familie von indizierenden Lösungen von $\dot{A}=\left[A^{2}, B\right], \quad \dot{B}=0$, so definieren die zugehörigen strikten formalen Laurentlösungen eine Hauptbalance von $\dot{A}=\left[A^{2}, B\right], \quad \dot{B}=0$. Diese beschreibt das asymptotische Verhalten von $\ddot{A} q u i v a l e n z k l a s s e n$ von Paaren von Matrizen in allgemeinen Punkten des verallgemeinerten Thetadivisors. 


\section{Kapitel 4}

\section{Fortsetzungen von holomorphen Schnitten}

Sei $(A, B) \in\left(\mathrm{gl}_{k} \times \mathrm{gl}_{k}\right) / G L_{k}$ so gewählt, dass die zugehörige Spektralkurve $S$ nichtsingulär ist. $S$ ist gegeben durch die Nullstellenmenge von $\psi=\operatorname{det}((A+$ $\left.\zeta B)+\eta \mathbb{1}_{k}\right) \in H^{0}\left(R, \pi_{R}^{*} \mathcal{O}_{\mathbb{P}^{1}}(k)\right)$. Wir erinnern an dieser Stelle an $m:=\frac{1}{2}(k-1)$. Der Schnitt $\psi$ ist von der Form $\psi=\eta^{2 m+1}+a_{1} \eta^{2 m}+a_{2} \eta^{2 m-1}+\ldots+a_{k}$ mit Schnitten $a_{i} \in H^{0}\left(\mathbb{P}^{1}, \mathcal{O}_{\mathbb{P}^{1}}(i)\right)$. Zum Beispiel ist $a_{1}=\operatorname{tr}(A+\zeta B)$ und $a_{k}=\operatorname{det}(A+\zeta B)$.

Mithilfe der Painlevé - Analysis aus Kapitel 3 können wir jede Funktion $f \in \mathbb{C}\left[\mathrm{gl}_{k} \times \mathrm{gl}_{k}\right]^{\mathrm{GL}_{k}}$ bis auf eine Konstante mit Thetafunktionen ausdrücken. In Kapitel 6 führen wir dies explizit durch, indem wir die in Kapitel 3 berechneten strikten formalen Laurentlösungen $A(z), B$, die das asymptotische Verhalten von Äquivalenzklassen von Paaren von Matrizen entlang $\beta$ in allgemeinen Punkten des Thetadivisors beschreiben, in die Funktion $f$ einsetzen. Zur Bestimmung des konstanten Terms betrachten wir das Verhalten von $f$ in einem konkreten Punkt des Thetadivisors $\Theta \subset J(S)$. Dieser konkrete Punkt ist gegeben durch $\mathcal{O}_{S}(m):=\pi_{S}^{*} \mathcal{O}_{\mathbb{P}^{1}}(m)=\pi_{S}^{*} \mathcal{O}_{\mathbb{P}^{1}}\left(\frac{1}{2}(k-1)\right) \in \Theta \subset J^{k+g-1}(S)$ (siehe Abschnitt 1.5).

In diesem Kapitel untersuchen wir daher einen Fluss $L^{z}(m)$ mit $L^{0}(m)=\mathcal{O}(m)$ in $J^{k+g-1}(S)$ definiert durch die tangentiale Richtung $\beta=\eta^{2} \zeta^{-1} \in H^{1}(S, \mathcal{O})$. Wir definieren ein Vektorbündel $V$ über $\mathbb{C}$, das den Fluss $L^{z}(m)$ parametrisiert und für das für $z \neq 0$ und $|z|$ hinreichend klein die Faser $V_{z}$ gleich $H^{0}\left(S, L^{z}(m)\right)$ ist.

Im Verlauf des Kapitels werden wir $V_{0}$ als Unterraum von $H^{0}(S, \mathcal{O}(m))$ genau beschreiben. $V_{0}$ besteht aus den Schnitten aus $H^{0}(S, \mathcal{O}(m))$, die sich, für $|z|$ hinreichend klein, zu Schnitten von $L^{z}(m)$ fortsetzen lassen. Diese Fortsetzungen werden wir konkret berechnen und in Kapitel 5 zur Bestimmung des 
asymptotischen Verhalten von $A(z), B(z)$ entlang $\beta$ durch $\mathcal{O}(m)$ verwenden.

\subsection{Definition des Vektorbündels $V$}

Seien wie in Abschnitt $2.1\left(U_{0}, \zeta\right)$ und $\left(U_{1}, \zeta^{\prime}\right)$ Karten von $\mathbb{P}^{1}, \pi:=\pi_{R}: R \rightarrow \mathbb{P}^{1}$ die Projektion vom Totalraum $R$ des Bündels $\mathcal{O}_{\mathbb{P}^{1}}(1)$ auf $\mathbb{P}^{1}$ und $\widetilde{U_{0}}=\pi^{-1}\left(U_{0}\right)$ bzw. $\widetilde{U_{1}}=\pi^{-1}\left(U_{1}\right)$. Seien $\left(\widetilde{U_{0}},(\eta, \zeta)\right)$ und $\left(\widetilde{U_{1}},\left(\eta^{\prime}, \zeta^{\prime}\right)\right)$ die in Abschnitt 2.1 definierten Karten von $R$. Insbesondere gilt $\zeta^{\prime}=\frac{1}{\zeta}$ bzw. $\eta^{\prime}=\frac{\eta}{\zeta}$ über $\widetilde{U_{0}} \cap \widetilde{U_{1}}$. Die jeweiligen Einschränkungen auf die Spektralkurve $S \subset R$ nennen wir wie in Abschnitt $2.1(U,(\eta, \zeta))$ bzw. $\left(U^{\prime},\left(\eta^{\prime}, \zeta^{\prime}\right)\right)$.

Wir definieren ein Vektorbündel $V \rightarrow \mathbb{C}$ wie folgt:

Sei $M$ das Geradenbündel über $\mathbb{C} \times S$ mit Faser $\left.M\right|_{(z, w)}=\left.L^{z}(m)\right|_{w}$ und $\pi: \mathbb{C} \times S \rightarrow \mathbb{C}$ die Projektion. Hierbei sei $L^{z}(m)$ definiert durch die Übergangsfunktion $g_{U, U^{\prime}}=\zeta^{m} \exp \left(z \frac{\eta^{2}}{\zeta}\right) \cdot \eta^{2} \zeta^{-1}$ ist Repräsentant von $\beta=\pi^{*} \alpha \in H^{1}(S, \mathcal{O})$ mit $\alpha \in H^{1}\left(\mathbb{P}^{1}, \mathcal{O}(-2)\right)$ repräsentiert durch $g_{U_{0} U_{1}}=\zeta^{-1}$ über $U_{0} \cap U_{1}$.

Anders als in Abschnitt 2.1 starten wir nun für $z=0$ in einem Punkt des Thetadivisors, wobei aufgrund von Abschnitt 2.5 für $z \neq 0$ mit $|z|$ hinreichend klein $L^{z}(m) \notin \Theta$ gilt, d.h. der Fluss den Thetadivisor sofort verlässt. Die direkte Bildgarbe $(\pi)_{*} M$ über $\mathbb{C}$ ist eine torsionsfreie Garbe. Nach dem Struktursatz für endlich erzeugte Moduln über Hauptidealringen ist $\pi_{*} M$ eine lokal freie Garbe, definiert also ein Vektorbündel $V \rightarrow \mathbb{C}$. Da das Geradenbündel $L^{z}(m)$ im Allgemeinen nicht im Thetadivisor $\Theta \subset J^{k+g-1}(S)$ liegt, hat für allgemeines $z$ das Bündel $L^{z}(m) \otimes \pi_{S}^{*} \mathcal{O}_{\mathbb{P}^{1}}(-1)$ keine globalen holomorphen Schnitte. Damit gilt $h^{0}\left(S, L^{z}(m)\right)=k$ für allgemeines $z \neq 0$. Es ist $\operatorname{rang} V=k$, und für eine allgemeine Faser $V_{z}, z \neq 0$, gilt $V_{z}=H^{0}\left(S, L^{z}(m)\right.$.

Wir wollen in diesem Kapitel $V_{0} \subset H^{0}(S, \mathcal{O}(m))$ genauer untersuchen. Wir berechnen also gerade die Schnitte von $\mathcal{O}(m):=\pi_{S}^{*} \mathcal{O}(m)$ über $S$, die sich lokal zu Schnitten von $L^{z}(m)$ über $S$ fortsetzen lassen, wobei lokal hier bedeutet, dass $z$ betragsmäßig hinreichend klein ist. Wir erinnern an dieser Stelle daran, dass wir in Satz 2.4

$$
H^{0}(S, \mathcal{O}(m))=\bigoplus_{i=0}^{m} \eta^{i} H^{0}\left(\mathbb{P}^{1}, \mathcal{O}(m-i)\right)
$$

sowie in Satz 2.6

$$
H^{1}(S, \mathcal{O}(m))=\bigoplus_{i=2}^{m} \eta^{m+i} H^{1}\left(\mathbb{P}^{1}, \mathcal{O}(-i)\right)
$$

gezeigt haben. 
Für $n \in \mathbb{Z}$ schreiben wir ab sofort $H^{0}\left(\mathbb{P}^{1}, \mathcal{O}(n)\right)$ für $\pi^{*} H^{0}\left(\mathbb{P}^{1}, \mathcal{O}_{\mathbb{P}^{1}}(n)\right)$, falls aus dem Zusammenhang klar ersichtlich ist, dass wir die zurückgezogenen holomorphen Schnitte von $\mathcal{O}_{S}(n)$ meinen. Ein möglicher Kandidat für die Faser $V_{0}$ ist $H^{0}\left(\mathbb{P}^{1}, \mathcal{O}(m)\right) \subset H^{0}(S, \mathcal{O}(m))$. Es gilt jedoch

$$
\operatorname{dim} H^{0}\left(\mathbb{P}^{1}, \mathcal{O}_{\mathbb{P}^{1}}(m)\right)=m+1=\frac{1}{2}(k-1)+1<k .
$$

Insbesondere ist $\operatorname{dim} V_{0}-\operatorname{dim} H^{0}\left(\mathbb{P}^{1}, \mathcal{O}(m)\right)=\frac{1}{2}(k-1)=m$. Wir zeigen in Satz 4.35, dass $V_{0}=H^{0}\left(\mathbb{P}^{1}, \mathcal{O}(m)\right) \oplus \eta H^{0}\left(\mathbb{P}^{1}, \mathcal{O}(m-1)\right)$ gilt.

\subsection{Holomorphe Fortsetzungen}

Für festes $z \in \mathbb{C}$ ist ein globaler holomorpher Schnitt $\widehat{s}(z) \in H^{0}\left(S, L^{z}(m)\right)$ gegeben durch holomorphe Abbildungen $s(z): U \rightarrow \mathbb{C}$ und $s^{\prime}(z): U^{\prime} \rightarrow \mathbb{C}$ mit $s(z)=\zeta^{m} \exp \left(z \frac{\eta^{2}}{\zeta}\right) s^{\prime}(z)$ über $U \cap U^{\prime}$. Suchen wir eine holomorphe Fortsetzung von $\widehat{s_{0}} \in H^{0}(S, \mathcal{O}(m))$ auf ein Gebiet um $0 \in \mathbb{C}$, so benötigen wir holomorph von $z$ abhängige Schnitte $\left.\widehat{s}(z) \in H^{0}\left(S, L^{z}(m)\right)\right)$ mit $\widehat{s}(0)=\widehat{s_{0}}$, d.h. holomorph von $z$ abhängige holomorphe Abbildungen $s(z): U \rightarrow \mathbb{C}$ und $s^{\prime}(z): U^{\prime} \rightarrow \mathbb{C}$ mit $s(z)=\zeta^{m} \exp \left(z \frac{\eta^{2}}{\zeta}\right) s^{\prime}(z)$ über $U \cap U^{\prime}$.

Es gibt genau dann eine holomorphe Fortsetzung von $\widehat{s_{0}}$ auf eine Umgebung von $0 \in \mathbb{C}$, falls es konvergente Potenzreihen in $z$ gibt mit

$$
\begin{gathered}
s(z)=s_{0}+z s_{1}+z^{2} s_{2}+\ldots \text { über } U \\
s^{\prime}(z)=s_{0}^{\prime}+z s_{1}^{\prime}+z^{2} s_{2}^{\prime}+\ldots \text { über } U^{\prime},
\end{gathered}
$$

die $s(z)=\zeta^{m} \exp \left(z \frac{\eta^{2}}{\zeta}\right) s^{\prime}(z)$ über $U \cap U^{\prime}$ erfüllen, wobei die $s_{i}$ bzw. $s_{i}^{\prime}$ holomorphe Funktionen $s_{i}: U \rightarrow \mathbb{C}$ bzw. $s_{i}^{\prime}: U^{\prime} \rightarrow \mathbb{C}$ sind.

Bemerkung 4.1. Untersuchen wir die einzelnen Koeffizienten vor den Potenzen von $z$, so erhalten wir, falls $s$ und $s^{\prime}$ eine Fortsetzung eines Schnittes $\widehat{s_{0}}$ aus $V_{0}$ definieren, über $U \cap U^{\prime}$ die Bedingungen

$$
\begin{aligned}
& s_{0}=\zeta^{m} s_{0}^{\prime}, \\
& s_{1}=\zeta^{m} \frac{\eta^{2}}{\zeta} s_{0}^{\prime}+\zeta^{m} s_{1}^{\prime},
\end{aligned}
$$

und allgemein

$$
s_{i}=\sum_{j=0}^{i} \eta^{2(i-j)} \zeta^{m-i+j} \frac{1}{(i-j) !} s_{j}^{\prime}
$$

an die Abbildungen $s_{i}$ und $s_{i}^{\prime}$. 
Diese Bedingungen bedeuten, dass jeder durch (4.1) definierte Kozykel

$$
s_{i}-\zeta^{m} s_{i}^{\prime}=\sum_{j=0}^{i-1} \eta^{2(i-j)} \zeta^{m-i+j} \frac{1}{(i-j) !} s_{j}^{\prime}
$$

über $U \cap U^{\prime}$ ein Repräsentant von $0 \in H^{1}(S, \mathcal{O}(m))$ sein muss. Schon für $i=1$ mit $s_{1}-\zeta^{m} s_{1}^{\prime}=\eta^{2} \zeta^{-1}\left(\zeta^{m} s_{0}^{\prime}\right)=\eta^{2} \zeta^{-1} s_{0}$ erhalten wir so einen Hinderungsgrund für die Existenz einer holomorphen Fortsetzung. Ist z.B. $\eta^{m} \widehat{p_{0}} \in \eta^{m} H^{0}\left(\mathbb{P}^{1}, \mathcal{O}\right)$ und $\widehat{p_{0}} \neq 0$, so ist $\beta \cdot \eta^{m} \widehat{p_{0}}=\eta^{2} \pi^{*} \alpha \cdot \eta^{m} \widehat{p_{0}} \in \eta^{m+2} H^{1}\left(\mathbb{P}^{1}, \mathcal{O}(-2)\right) \subset H^{1}(S, \mathcal{O}(m))$ ungleich null und wird durch $\eta^{m+2} \zeta^{-1} p_{0}$ über $U \cap U^{\prime}$ repräsentiert. Ein solches $\widehat{s_{0}}=\eta^{m} \widehat{p_{0}}$ kann somit keine holomorphe Fortsetzung zu Schnitten $\widehat{s(z)} \in$ $H^{0}\left(S, L^{z}(m)\right)$ besitzen.

\subsection{Fortsetzungen endlicher Ordnung}

Will man eine Fortsetzung eines Schnitts $\widehat{s_{0}}$ konstruieren, so bietet es sich an, dies schrittweise durchzuführen:

Definition 4.2. Sei $l \in \mathbb{N}$. Eine Fortsetzung eines Schnittes $\widehat{s_{0}}$ von $\mathcal{O}(m)$ über $S$ zu einem Schnitt von $L^{z}(m)$ zur l-ten Ordnung sei definiert durch holomorphe Funktionen

$$
\begin{array}{rlrl}
s:=s(z) & =s_{0}+z s_{1}+z^{2} s_{2}+\ldots+z^{l} s_{l}, & & s_{i} \in H^{0}(U, \mathcal{O}), \\
s^{\prime}:=s^{\prime}(z) & =s_{0}^{\prime}+z s_{1}^{\prime}+z^{2} s_{2}^{\prime}+\ldots+z^{l} s_{l}^{\prime}, & s_{i}^{\prime} \in H^{0}\left(U^{\prime}, \mathcal{O}\right),
\end{array}
$$

mit $s=\zeta^{m} \exp \left(z \frac{\eta^{2}}{\zeta}\right) s^{\prime} \bmod z^{l+1}$ über $U \cap U^{\prime}$.

Die holomorphen Funktionen $s_{i} \in \mathcal{O}(U)$ bzw. $s_{i}^{\prime} \in \mathcal{O}\left(U^{\prime}\right)$ für $0 \leq i \leq l \mathrm{zu}$ Fortsetzungen zur $l$-ten Ordnung müssen ebenfalls Gleichung (4.1) erfüllen.

Fortsetzungen von Schnitten von $\mathcal{O}(m)$ zur $l$-ten Ordnung sind Schnitte des folgenden Vektorbündels vom Rang $l+1$ über $S$ :

Definition 4.3. Sei $F^{l}(m)$ das Vektorbündel vom Rang $l+1$ über $S$ definiert durch die Übergangsfunktion $g_{U U^{\prime}}:=\widetilde{M^{l}(m)}$ mit

$$
\left(\widetilde{M^{l}(m)}\right)_{i j}:= \begin{cases}\frac{1}{(i-j) !} \eta^{2(i-j)} \zeta^{m-(i-j)} & \text { für } 0 \leq j \leq i \leq l \\ 0 & \text { sonst. }\end{cases}
$$

Ein globaler holomorpher Schnitt $\widehat{s}$ von $F^{l}(m)$ ist dann definiert durch jeweils $l+1$ holomorphe Funktionen $s_{i}: U \rightarrow \mathbb{C}$ bzw. $s_{i}^{\prime}: U^{\prime} \rightarrow \mathbb{C}, 0 \leq i \leq l$, mit

$$
\widetilde{M^{l}(m)}\left(\begin{array}{c}
s_{0}^{\prime} \\
\vdots \\
s_{l}^{\prime}
\end{array}\right)=\left(\begin{array}{c}
s_{0} \\
\vdots \\
s_{l}
\end{array}\right) \text { über } U \cap U^{\prime} .
$$


Für alle $0 \leq i \leq l$ muss folglich

$$
s_{i}=\sum_{j=0}^{l}\left(\widetilde{M^{l}(m)}\right)_{i, j} \cdot s_{j}^{\prime}=\sum_{j=0}^{i} \eta^{2(i-j)} \zeta^{m-i+j} \frac{1}{(i-j) !} s_{j}^{\prime}
$$

über $U \cap U^{\prime}$ gelten. Für einen Schnitt $\widehat{s} \in H^{0}\left(S, F^{l}(m)\right)$ repräsentieren die ersten Einträge $s_{0}, s_{0}^{\prime}$ einen Schnitt $\widehat{s_{0}}$ aus $H^{0}(S, \mathcal{O}(m))$. Somit stellt $\widehat{s}$ eine Fortsetzung von $\widehat{s_{0}}$ zur Ordnung $l$ dar.

In $\exp \left(z \frac{\eta^{2}}{\zeta}\right)$ kommen nur gerade Potenzen von $\eta$ vor. Besonders einfache Fortsetzungen von Schnitten $s_{0}$ aus $H^{0}\left(\mathbb{P}^{1}, \mathcal{O}(m)\right) \subset H^{0}(S, \mathcal{O}(m))$ zur Ordnung $l$ sind daher:

Definition 4.4. Die spezielle Fortsetzung eines $\widehat{s_{0}} \in \pi^{*} H^{0}\left(\mathbb{P}^{1}, \mathcal{O}(m)\right)$ zur Ordnung l sei definiert durch Funktionen

$$
\begin{aligned}
s & =s_{0}+z \eta^{2} s_{1}+\cdots+z^{l} \eta^{2 l} s_{l} & & \text { über } U \\
s^{\prime} & =s_{0}^{\prime}+z \eta^{\prime 2} s_{1}^{\prime}+\cdots+z^{l} \eta^{\prime 2 l} s_{l}^{\prime} & & \text { über } U^{\prime}
\end{aligned}
$$

mit $s=\zeta^{m} \exp \left(z \frac{\eta^{2}}{\zeta}\right) s^{\prime} \bmod z^{l+1}$ über $U \cap U^{\prime}$, wobei die einzelnen $s_{i}$, $s_{i}^{\prime}$ unabhängig von $\eta$ und $\eta^{\prime}$ seien.

Spezielle Fortsetzungen zur Ordnung $l$ können ganz analog auch für Schnitte eines Vektorbündels $\mathcal{O}(n)$ für beliebiges $n \in \mathbb{N}$ definiert werden. Wir werden in Abschnitt 4.8 sehen, dass spezielle Fortsetzungen eines Schnittes $\widehat{s_{0}} \in H^{0}\left(\mathbb{P}^{1}, \mathcal{O}(n)\right)$ zur Ordnung $l$ globalen holomorphen Schnitten eines Vektorbündels $E^{l}(n)$ über $\mathbb{P}^{1}$ entsprechen. Diese Bündel definieren wir im folgenden Abschnitt.

\subsection{Yoneda-Erweiterungen über $\mathbb{P}^{1}$}

Sei wie in Abschnitt 4.1 das Element $0 \neq \alpha \in H^{1}\left(\mathbb{P}^{1}, \mathcal{O}(-2)\right)$ repräsentiert durch den Kozykel $\zeta^{-1}$ über $U_{0} \cap U_{1}$, wir schreiben hierfür $\alpha=\zeta^{-1}$. $\alpha \in H^{1}\left(\mathbb{P}^{1}, \mathcal{O}_{\mathbb{P}^{1}}(-2)\right)=\operatorname{Ext}^{1}\left(\mathcal{O}_{\mathbb{P}^{1}}, \mathcal{O}_{\mathbb{P}^{1}}(-2)\right)$ repräsentiert eine exakte Sequenz

$$
0 \rightarrow \mathcal{O}_{\mathbb{P}^{1}}(-2) \rightarrow E^{1} \rightarrow \mathcal{O}_{\mathbb{P}^{1}} \rightarrow 0
$$

von Vektorbündeln über $\mathbb{P}^{1}$. Da $\alpha=\zeta^{-1}$ gilt und Übergangsfunktionen $g_{U_{0} U_{1}}$ von $\mathcal{O}_{\mathbb{P}^{1}}$ durch 1 bzw. von $\mathcal{O}_{\mathbb{P}^{1}}(-2)$ durch $\zeta^{-2}$ über $U_{0} \cap U_{1}$ gegeben sind, ist das Vektorbündel $E^{1}$ vom Rang zwei definiert durch die Übergangsfunktion

$$
g_{U_{0}, U_{1}}=\left(\begin{array}{cc}
1 & 0 \\
\zeta^{-1} & \zeta^{-2}
\end{array}\right) .
$$


Durch Twisten mit einem Geradenbündel $\mathcal{O}_{\mathbb{P}^{1}}(n), n \in \mathbb{N}$, erhalten wir die exakte Sequenz

$$
0 \rightarrow \mathcal{O}_{\mathbb{P}^{1}}(n-2) \rightarrow E^{1}(n) \rightarrow \mathcal{O}_{\mathbb{P}^{1}}(n) \rightarrow 0
$$

von Vektorbündeln über $\mathbb{P}^{1} . E^{1}(n)$ ist durch die Übergangsfunktion

$$
g_{U_{0} U_{1}}=\left(\begin{array}{cc}
\zeta^{n} & 0 \\
\zeta^{n-1} & \zeta^{n-2}
\end{array}\right)=: M^{1}(n)
$$

definiert.

Definition 4.5. Seien $l, n \in \mathbb{N}$. Sei $E^{l}(n)$ das Vektorbündel vom Rang $l+1$ über $\mathbb{P}^{1}$ definiert durch die Übergangsfunktion $g_{U_{0} U_{1}}=M^{l}(n)$ mit

$$
\left(M^{l}(n)\right)_{i j}:= \begin{cases}\frac{1}{(i-j) !} \zeta^{n-i-j} & \text { für } 0 \leq j \leq i \leq l \\ 0 & \text { sonst. }\end{cases}
$$

Wir verallgemeinern nun $\alpha \in H^{1}\left(\mathbb{P}^{1}, \mathcal{O}(-2)\right)$ :

Definition 4.6. Sei $l \in \mathbb{N}$. Dann definieren wir

$$
\alpha_{(l)}:=\underbrace{\left(\frac{(-1)^{l}}{(l+1) !} \zeta^{-(l+1)} \ldots \zeta^{-1}\right)}_{l+1 \text { Stellen }} \in \mathcal{O}\left(U_{0} \cap U_{1}\right)^{\oplus(l+1)} .
$$

Die Übergangsmatrix $M^{l}(n)$ ist somit von der Form

$$
M^{l}(n)=\left(\begin{array}{ccccc}
\zeta^{n} & 0 & \ldots & \ldots & 0 \\
\zeta^{n-1} & \zeta^{n-2} & 0 & & \\
\frac{1}{2} \zeta^{n-2} & \zeta^{n-3} & \zeta^{n-4} & & \vdots \\
\vdots & & & \ddots & 0 \\
\frac{1}{l !} \zeta^{n-l} & \frac{\zeta^{n-l-1}}{(l-1) !} & \ldots & & \zeta^{n-2 l}
\end{array}\right)=\left(\begin{array}{c|c} 
& 0 \\
M^{l-1}(n) & \vdots \\
& 0 \\
\hline \alpha_{(l-1)} \cdot M^{l-1}(n) & \zeta^{n-2 l}
\end{array}\right) .
$$

Damit folgt

Satz 4.7. Für $l \in \mathbb{N}$ ist $\alpha_{(l-1)}$ Repräsentant eines Kozykels aus dem Raum $H^{1}\left(\mathbb{P}^{1},\left(E^{l-1}(n)\right)^{\vee} \otimes \mathcal{O}(n-2 l)\right) \cong \operatorname{Ext}^{1}\left(E^{l-1}(n), \mathcal{O}(n-2 l)\right)$, der die exakte Sequenz von Vektorbündeln

$$
0 \rightarrow \mathcal{O}_{\mathbb{P}^{1}}(n-2 l) \rightarrow E^{l}(n) \rightarrow E^{l-1}(n) \rightarrow 0
$$


darstellt. Für die zugehörige lange exakte Sequenz von globalen holomorphen Schnitten

$$
\begin{aligned}
& 0 \rightarrow H^{0}\left(\mathbb{P}^{1}, \mathcal{O}(n-2 l)\right) \rightarrow H^{0}\left(\mathbb{P}^{1}, E^{l}(n)\right) \rightarrow H^{0}\left(\mathbb{P}^{1}, E^{l-1}(n)\right) \\
& \quad \stackrel{\delta}{\rightarrow} H^{1}\left(\mathbb{P}^{1}, \mathcal{O}(n-2 l)\right) \rightarrow \ldots
\end{aligned}
$$

ist der Verbindungshomomorphismus $\delta$ gleich $\alpha_{(l-1)}$, d.h. definiert durch die Multiplikation mit $\alpha_{(l-1)} \in H^{1}\left(\mathbb{P}^{1},\left(E^{l-1}(n)\right)^{\vee} \otimes \mathcal{O}(n-2 l)\right)$.

\subsection{Eigenschaften von $E^{l}(n)$}

Für $l=n$ gilt

Satz 4.8. Das Vektorbündel $E^{n}(n)$ über $\mathbb{P}^{1}$ ist trivial.

Beweis: Nach dem Satz von Birkhoff-Grothendieck ist jedes Vektorbündel über $\mathbb{P}^{1}$ eine direkte Summe von Geradenbündeln. Wir stellen $E^{n}(n)$ als eine solche direkte Summe dar, indem wir die Übergangsmatrix $M^{n}(n)$ in eine Diagonalmatrix $X M^{n}(n) Y^{-1}$ mit $X \in \operatorname{GL}(n+1, \mathbb{C}[\zeta])$ und $Y \in \operatorname{GL}(n+$ $\left.1, \mathbb{C}\left[\zeta^{-1}\right]\right)$ transformieren. Sind die Diagonaleinträge dieser Matrix konstante komplexe Zahlen, so ist $E^{n}(n)$ trivial.

Zunächst vertauschen wir die Zeilen der Matrix $M^{n}(n)$ und erhalten die Matrix

$$
M_{n}(\zeta):=\left(\begin{array}{cccccc}
\frac{1}{(n) !} & \frac{\zeta^{-1}}{(n-1) !} & \frac{\zeta^{-2}}{(n-2) !} & & \ldots & \zeta^{-n} \\
\frac{\zeta^{1}}{(n-1) !} & \frac{1}{(n-2) !} & \frac{\zeta^{-1}}{(n-3) !} & & \zeta^{2-n} & 0 \\
\frac{\zeta^{2}}{(n-2) !} & \frac{\zeta}{(n-3) !} & \frac{1}{(n-4) !} & \ldots & 0 & 0 \\
\vdots & & & & & \\
\zeta^{n} & 0 & & \ldots & & 0
\end{array}\right)
$$

Diese definiert als Übergangsmatrix ebenfalls das Vektorbündel $E^{n}(n)$ über $\mathbb{P}^{1} . M_{n}(\zeta)$ ist eine pseudosymmetrische Matrix. Pseudosymmetrisch bedeutet hierbei, dass $M_{n}(\zeta)^{t}=M_{n}\left(\zeta^{-1}\right)$ gilt. Aufgrund dieser Symmetrieeigenschaft sind alle Minoren Minor $(0)=\frac{1}{n !}, \operatorname{Minor}(1)=-\frac{1}{n !(n-1) !}, \ldots, \operatorname{Minor}(n)=$ $\operatorname{det}\left(M_{n}(\zeta)\right)$ von $M_{n}(\zeta)$ konstante komplexe Zahlen ungleich null. Induktiv lässt sich nun zeigen, dass es Matrizen $X \in \mathrm{GL}(n+1, \mathbb{C}[\zeta])$ und $Y \in \mathrm{GL}(n+$ 
$\left.1, \mathbb{C}\left[\zeta^{-1}\right]\right)$ gibt mit

$$
X M_{n}(\zeta) Y^{-1}=\left(\begin{array}{ccccc}
\frac{1}{n !} & & & & \\
& \frac{\operatorname{Minor}(1)}{\operatorname{Minor}(0)} & & & \\
& & \frac{\operatorname{Minor}(2)}{\operatorname{Minor}(1)} & & \\
& & & \ddots & \\
& & & & \frac{\operatorname{det}(M)}{\operatorname{Minor}(n-1)}
\end{array}\right) .
$$

Hierbei ist $X=\left(Y^{-1}\right)^{t}\left(\zeta^{-1}\right)$.

Die einzelnen Minoren Minor $(i)$ und damit auch die Diagonaleinträge von $X M_{n}\left(\zeta^{-1}\right) Y^{-1}$ hängen nicht von $\zeta$ bzw. $\zeta^{-1}$ ab. Somit ist $E^{n}(n)$ gleich der direkten Summe $\mathcal{O}_{\mathbb{P} 1}^{\oplus(n+1)}$.

Folgerung 4.9. Für $l, n \in \mathbb{N}$ ist $E^{l}(n)=E^{l}(l) \otimes \mathcal{O}_{\mathbb{P}^{1}}(n-l)$ von der Form $\mathcal{O}_{\mathbb{P}^{1}}(n-l)^{\oplus(l+1)}$.

Folgerung 4.10. Für $l>n$ hat das Vektorbündel $E^{l}(n)$ über $\mathbb{P}^{1}$ keine nichttrivialen globalen holomorphen Schnitte.

\subsection{Kanonische Erweiterungen}

Wir erinnern an dieser Stelle daran, dass wir globale holomorphe Schnitte eines Vektorbündels $\mathcal{O}_{\mathbb{P}^{1}}(n)$ über $\mathbb{P}^{1}$ durch ihre Repräsentanten $s \in \mathcal{O}\left(U_{0}\right)$ über der offenen Menge $U_{0}$ darstellen. Z.B. beschreibt $\zeta^{j}$ mit $0 \leq j \leq n$ den Schnitt $\widehat{s} \in H^{0}\left(\mathbb{P}^{1}, \mathcal{O}_{\mathbb{P}^{1}}(n)\right)$ mit $s=\zeta^{j}, s^{\prime}=\left(\zeta^{\prime}\right)^{n-j}$ und $s=\zeta^{n} s^{\prime}$ über $U_{0} \cap U_{1}$. Diese Beschreibung hängt von der Wahl der Übergangsmatrix $g_{U_{0} U_{1}}=\zeta^{n}$ des Bündels $\mathcal{O}_{\mathbb{P}^{1}}(n)$ ab.

Elemente aus $H^{1}\left(\mathbb{P}^{1}, \mathcal{O}_{\mathbb{P}^{1}}(n)\right)$ sind durch ihre Repräsentanten aus $\mathcal{O}\left(U_{0} \cap U_{1}\right)$ definiert. Z.B. ist eine Basis von $H^{1}\left(\mathbb{P}^{1}, \mathcal{O}(-n)\right)$ gegeben durch die Repräsentanten $\zeta^{-n+j}$ mit $1 \leq j \leq n-1$.

Analog sind globale holomorphe Schnitte von $E^{l}(n)$ für $l \leq n$ definiert durch ihre Repräsentanten $s=\left(\begin{array}{lll}s_{0} & \ldots & s_{l}\end{array}\right)^{t}$ über $U_{0} . s$ steht dann für ein Paar $s$ über $U_{0}, s^{\prime}$ über $U_{1}$ mit $s=M^{l}(n) s^{\prime}$ über $U_{0} \cap U_{1}$. Wie für Geradenbündel $\mathcal{O}_{\mathbb{P}^{1}}(n)$ sind die möglichen Einträge $s_{i}$ von $s$ durch das Bündel $E^{l}(n)$ bestimmt. Diese Beschreibung hängt ebenfalls von der Wahl einer Übergangsmatrix $g_{U_{0} U_{1}}=M^{l}(n)$ ab.

Analog zu Erweiterungen von Schnitten von $\mathcal{O}_{S}(n)$ zur Ordnung $l$ definieren wir über $\mathbb{P}^{1}$ : 
Definition 4.11. Seien $n, l \in \mathbb{N}$ mit $l \leq n$. Ein Schnitt $s \in H^{0}\left(\mathbb{P}^{1}, E^{l}(n)\right)$, für den der erste Eintrag $s_{0}$ über $U_{0}$ den Schnitt $\widehat{s_{0}} \in H^{0}\left(\mathbb{P}^{1}, \mathcal{O}(n)\right)$ definiert, heißt Fortsetzung von $\widehat{s_{0}}$ zur Ordnung $l$.

Betrachten wir zu einem Schnitt $s \in H^{0}\left(\mathbb{P}^{1}, E^{l}(n)\right)$ nur den durch den ersten Eintrag $s_{0}$ definierten Schnitt $\widehat{s_{0}} \in H^{0}\left(\mathbb{P}^{1}, \mathcal{O}_{\mathbb{P}^{1}}(n)\right)$, so entspricht dies der Projektionsabbildung $E^{l}(n) \stackrel{\mathrm{pr}_{0}}{\longrightarrow} \mathcal{O}_{\mathbb{P}^{1}}(n)$. Zu einem festen Schnitt $\widehat{s_{0}} \in$ $H^{0}\left(\mathbb{P}^{1}, \mathcal{O}_{\mathbb{P}^{1}}(n)\right)$ gibt es möglicherweise viele verschiedene Erweiterungen zur Ordnung $l$. Z.B. ist für $l=1$ die zu (4.3), der Sequenz

$$
0 \rightarrow \mathcal{O}_{\mathbb{P}^{1}}(n-2 l) \rightarrow E^{l}(n) \rightarrow E^{l-1}(n) \rightarrow 0,
$$

gehörige lange exakte Sequenz von der Form

$$
\begin{aligned}
0 & \rightarrow H^{0}\left(\mathbb{P}^{1}, \mathcal{O}_{\mathbb{P}^{1}}(n-2)\right) \rightarrow H^{0}\left(\mathbb{P}^{1}, E^{1}(n)\right) \stackrel{\mathrm{pr}_{0}}{\rightarrow} H^{0}\left(\mathbb{P}^{1}, \mathcal{O}_{\mathbb{P}^{1}}(n)\right) \\
& \stackrel{\alpha}{\rightarrow} H^{1}\left(\mathbb{P}^{1}, \mathcal{O}_{\mathbb{P}^{1}}(n-2)\right) \rightarrow \ldots
\end{aligned}
$$

Ist hier $n>0$, so ist für beliebiges $\widehat{s_{0}} \in H^{0}\left(\mathbb{P}^{1}, \mathcal{O}(n)\right)$ das $\operatorname{Urbild~}_{\operatorname{pr}_{0}^{-1}}\left(\widehat{s_{0}}\right)$ von der Dimension $h^{0}\left(\mathbb{P}^{1}, \mathcal{O}_{\mathbb{P}^{1}}(n-2)\right)$. Für $n=0$ ist $\alpha$ ein Isomorphismus und $H^{0}\left(\mathbb{P}^{1}, E^{1}\right)=0$.

Allgemein ist der Kern der Projektionsabbildung $E^{l}(n) \stackrel{\mathrm{pr}_{0}}{\rightarrow} \mathcal{O}_{\mathbb{P}^{1}}(n)$ das Vektorbündel vom Rang $l$ mit Übergangsfunktion $g_{U_{0} U_{1}}=M^{(l-1)}(n-2)$ mit

$$
\left(\begin{array}{cccc}
\zeta^{n-2} & 0 & \cdots & 0 \\
\zeta^{n-3} & \zeta^{n-4} & \ddots & \vdots \\
\vdots & & \ddots & 0 \\
\frac{\zeta^{n-l-1}}{(l-1) !} & \cdots & & \zeta^{n-2 l}
\end{array}\right)=M^{(l-1)}(n-2)
$$

Wir erhalten die exakte Sequenz von Vektorbündeln

$$
0 \rightarrow E^{l-1}(n-2) \rightarrow E^{l}(n) \stackrel{\mathrm{pr}_{0}}{\rightarrow} \mathcal{O}_{\mathbb{P}^{1}}(n) \rightarrow 0
$$

über $\mathbb{P}^{1}$.

Definition und Satz 4.12. Sei $n \in \mathbb{N}$. Dann gibt es zu jedem Schnitt $\widehat{s_{0}} \in H^{0}\left(\mathbb{P}^{1}, \mathcal{O}_{\mathbb{P}^{1}}(n)\right)$ eine eindeutige Fortsetzung $s \in H^{0}\left(\mathbb{P}^{1}, E^{n}(n)\right)$ zur Ordnung $n$. Diese Fortsetzung nennen wir kanonische Fortsetzung.

Beweis: Mit Folgerung 4.10 erhalten wir für $l=n$ zur Sequenz (4.4) die lange exakte Sequenz

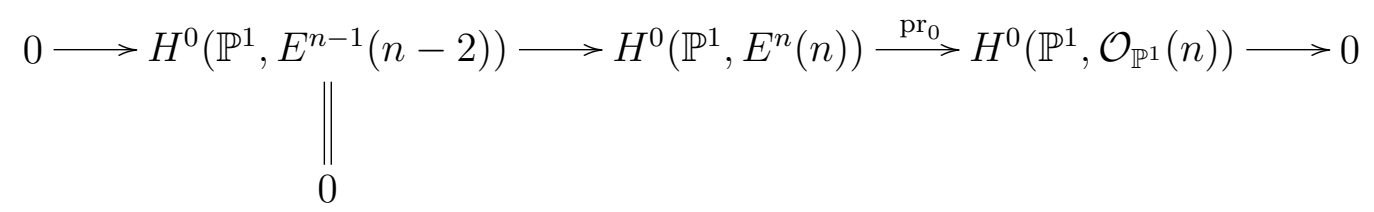


Somit ist $\mathrm{pr}_{0}$ ein Isomorphismus.

Im folgenden Satz geben wir zu jedem Schnitt $\widehat{s_{0}} \in H^{0}\left(\mathbb{P}^{1}, \mathcal{O}_{\mathbb{P}^{1}}(n)\right)$ die kanonische Erweiterung in $H^{0}\left(\mathbb{P}^{1}, E^{n}(n)\right)$ an:

Satz 4.13. Für $\zeta^{j} \in H^{0}\left(\mathbb{P}^{1}, \mathcal{O}_{\mathbb{P}^{1}}(n)\right)$ mit $0 \leq j \leq n$ beschreibt der $n+1$ dimensionale Vektor

$$
b_{n, n}(j):=\left(\begin{array}{c}
\zeta^{j} \\
\frac{j}{n} \zeta^{j-1} \\
\vdots \\
f_{i, n}(j) \zeta^{j-i} \\
\vdots \\
f_{n, n}(j)
\end{array}\right) \text { über } U_{0}
$$

mit

$$
f_{i, n}(j):= \begin{cases}\frac{j !(n-i) !}{i !(j-i) ! n !} & \text { falls } 0 \leq i \leq j \\ 0 & \text { sonst }\end{cases}
$$

die kanonische Erweiterung von $\zeta^{j}$.

Beweis: Wir müssen lediglich zeigen, dass $b_{n, n}(j)$ einen globalen holomorphen Schnitt von $E^{n}(n)$ bezüglich der Übergangsmatrix $M^{n}(n)$ beschreibt. Die Projektion $p r_{0}$ von $b_{n, n}(j)$ ist dann automatisch gleich dem Schnitt $\zeta^{j}$.

Beschreiben allgemein $s: U_{0} \rightarrow \mathbb{C}$ bzw. $t: U_{1} \rightarrow \mathbb{C}$ mit $s=\zeta^{n} t$ über $U_{0} \cap U_{1}$ einen globalen holomorphen Schnitt von $\mathcal{O}_{\mathbb{P}^{1}}(n)$, so gilt für die Ableitungen nach $\zeta$ über $U_{0} \cap U_{1}$ :

$$
\begin{aligned}
s^{\prime} & =n \zeta^{n-1} t-\zeta^{n-2} t^{\prime} \\
s^{\prime \prime} & =n(n-1) \zeta^{n-2} t-2(n-1) \zeta^{n-3} t^{\prime}+\zeta^{n-4} t^{\prime \prime}
\end{aligned}
$$

( $t$ ist eine Funktion in $\zeta^{\prime}$, und $\zeta^{\prime}=\zeta^{-1}$ über $U_{0} \cap U_{1}$ ). Allgemein gilt für die $i$-te Ableitung mit $i \leq n$

$$
s^{(i)}=\sum_{c=0}^{i}\left(\begin{array}{l}
i \\
c
\end{array}\right) \frac{(n-c) !}{(n-i) !}(-1)^{c} \zeta^{n-i-c} t^{(c)}
$$

über $U_{0} \cap U_{1}$. 
Ist insbesondere $s=\zeta^{j}$, so definieren für $l \leq n$

$$
\left(\begin{array}{c}
s \\
s^{\prime} \\
\vdots \\
s^{(i)} \\
\vdots \\
s^{(l)}
\end{array}\right)=\left(\begin{array}{c}
\zeta^{j} \\
j \zeta^{j-1} \\
\vdots \\
\prod_{q=0}^{i-1}(j-q) \zeta^{j-i} \\
\vdots \\
\left(\frac{d^{l}}{d \zeta}\right)\left(\zeta^{j}\right)
\end{array}\right)
$$

und das zugehörige $\left(\begin{array}{c}t \\ \vdots \\ t^{(l)}\end{array}\right)$ einen globalen holomorphen Schnitt des Vektorbündels $\widetilde{E^{l}(n)}$ über $\mathbb{P}^{1}$ definiert durch die Übergangsfunktion $g_{U_{0} U_{1}}=N^{l}(n)$ mit

$$
N^{l}(n)=\left(\begin{array}{cccc}
\zeta^{n} & 0 & \ldots & 0 \\
n \zeta^{n-1} & -\zeta^{n-2} & \ddots & \vdots \\
\vdots & & \ddots & 0 \\
\frac{n !}{(n-l) !} \zeta^{n-l} & \ldots & \ldots & (-1)^{l} \zeta^{n-2 l}
\end{array}\right)
$$

wobei

$$
\left(N^{l}(n)\right)_{i c}= \begin{cases}\left(\begin{array}{l}
i \\
c
\end{array}\right) \frac{(n-c) !}{(n-i) !}(-1)^{c} \zeta^{n-i-c} & \text { für } 0 \leq c \leq i \leq l \\
0 & \text { sonst }\end{cases}
$$

gilt. $\widetilde{E^{l}(n)}$ ist gleich dem Vektorbündel $E^{l}(n)$, denn mit der $(l+1) \times(l+1)$ Diagonalmatrix $X(l)$ mit $X(l)_{i c}=i ! \prod_{q=0}^{i-1}(n-q) \delta_{i c}$ und $\operatorname{der}(l+1) \times(l+1)$ Diagonalmatrix $Y(l)$ mit $Y(l)_{i c}=(-1)^{c} \delta_{i c}$ gilt für $c>i$

$$
\begin{aligned}
\left(X(l) M^{l}(n)\right)_{i c} & =0 \\
& =\left(N^{l}(n) Y(l) X(l)\right)_{i c}
\end{aligned}
$$


und für $c \leq i$ :

$$
\begin{aligned}
\left(X(l) M^{l}(n)\right)_{i c} & =X(l)_{i i}\left(M^{l}(n)\right)_{i c} \\
& =i ! \prod_{q=0}^{i-1}(n-q) \cdot \frac{1}{(i-c) !} \zeta^{n-i-c} \\
& =\frac{i !}{c !(i-c) !} \cdot \frac{(n-c) !}{(n-i) !} \cdot c ! \prod_{q=0}^{c-1}(n-q) \zeta^{n-i-c} \\
& =\left(N^{l}(n)\right)_{i c} \cdot(-1)^{c} X(l)_{c c} \\
& =\left(N^{l}(n) Y(l) X(l)\right)_{i c} .
\end{aligned}
$$

Damit ist $X(l) M^{l}(n) X(l)^{-1} Y(l)^{-1}=N^{l}(n)$ und

$$
X(l)^{-1}\left(\begin{array}{c}
\zeta^{j} \\
\vdots \\
\prod_{q=0}^{i-1}(j-q) \zeta^{j-i} \\
\vdots \\
\frac{d^{l}}{d \zeta}\left(\zeta^{j}\right)
\end{array}\right)
$$

definiert einen Schnitt von $E^{l}(n)$ bezüglich der Übergangsmatrix $M^{l}(n)$. Mit

$$
\begin{aligned}
X(n)^{-1}\left(\begin{array}{c}
\zeta^{j} \\
\vdots \\
\frac{d^{n}}{d \zeta^{\prime}}\left(\zeta^{j}\right)
\end{array}\right) & =\left(\begin{array}{c}
\zeta^{j} \\
\vdots \\
\frac{\prod_{q=0}^{i-1}(j-q)}{i ! \prod_{q=0}^{i-1}(n-q)} \zeta^{j-i} \\
\vdots \\
\zeta^{j} \\
\vdots \\
f_{i, n}(j) \zeta^{j-i} \\
\vdots
\end{array}\right) \\
& =b_{n, n}(j)
\end{aligned}
$$

folgt die Behauptung. 
Bemerkung 4.14. Einen anderen Beweis zu Satz 4.13 erhält man, indem man für den über $U_{1}$ definierten Vektor

$$
b_{n, n}^{\prime}(j)=\left(\begin{array}{c}
\left(\zeta^{\prime}\right)^{n-j} \\
\vdots \\
(-1)^{i} f_{i, n}(n-j)\left(\zeta^{\prime}\right)^{n-j-i} \\
\vdots \\
(-1)^{n} f_{n, n}(n-j)
\end{array}\right)
$$

nachrechnet, dass über $U_{0} \cap U_{1}$ gilt $M^{l}(n) b_{n, n}^{\prime}(j)=b_{n, n}(j)$. Wir haben den Beweis dort so geführt, weil dieser explizit die Gleichheit des Jetbündels $J_{l}(n)$ mit $E^{l}(n)$ für $l \leq n$ zeigt.

\section{Jetbündel}

Das durch die Übergangsfunktion $g_{U_{0} U_{1}}=N^{l}(n)$ definierte Vektorbündel vom Rang $l+1$ über $\mathbb{P}^{1}$ ist das sogenannte $l$-te Jetbündel $J_{l}\left(\mathcal{O}_{\mathbb{P}^{1}}(n)\right)$ zu $\mathcal{O}_{\mathbb{P}^{1}}(n)$. Für $l, n \in \mathbb{N}$ ist die Sequenz

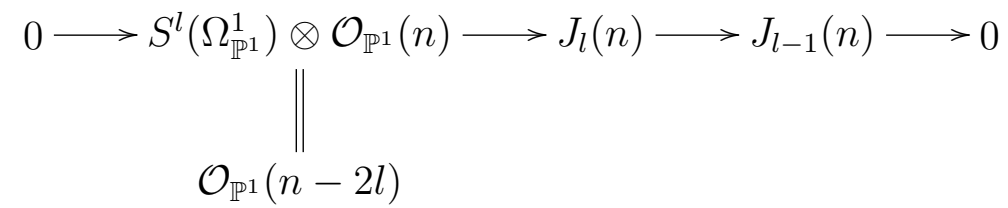

exakt. Für $l \leq n$ ist $J_{l}\left(\mathcal{O}_{\mathbb{P}^{1}}(n)\right)$ gleich $\mathcal{O}_{\mathbb{P}^{1}}(n-l)^{\oplus(l+1)}$ (zu Jetbündeln siehe z.B. [DRS01]).

\subsection{Kanonische Erweiterungen in $H^{0}\left(\mathbb{P}^{1}, E^{l}(n)\right)$}

Seien $c, l, n \in \mathbb{N}$. Die Projektionsabbildung $\operatorname{pr}_{0}: E^{l}(n) \rightarrow \mathcal{O}_{\mathbb{P}^{1}}(n)$ können wir für $0 \leq c \leq l$ verallgemeinern zur Projektionsabbildung $\operatorname{pr}_{c}: E^{l}(n) \rightarrow E^{c}(n)$. Auch hier ist der Kern ein Vektorbündel. Dieses ist vom Rang $l-c$ und ist definiert durch die Übergangsfunktion

$$
\left(\begin{array}{cccc}
\zeta^{n-2-2 c} & 0 & \cdots & 0 \\
\zeta^{n-3-2 c} & \zeta^{n-4-2 c} & \ddots & \vdots \\
\vdots & & \ddots & 0 \\
\frac{\zeta^{n-l-1-c}}{(l-1-c) !} & \cdots & & \zeta^{n-2 l}
\end{array}\right)=M^{l-1-c}(n-2(c+1)) .
$$


Wir erhalten die exakte Sequenz von Vektorbündeln

$$
0 \rightarrow E^{l-c-1}(n-2(c+1)) \rightarrow E^{l}(n) \stackrel{\mathrm{pr}_{c}}{\longrightarrow} E^{c}(n) \rightarrow 0
$$

über $\mathbb{P}^{1}$. Für $l=n$ liefert die zugehörige lange exakte Sequenz eine injektive Abbildung

$$
H^{0}\left(\mathbb{P}^{1}, E^{n}(n)\right) \stackrel{\mathrm{pr}_{c}}{\rightarrow} H^{0}\left(\mathbb{P}^{1}, E^{c}(n)\right) .
$$

Definition 4.15. Seien $l, n \in \mathbb{N}$ und $l \leq n$. Sei $\widehat{s_{0}} \in H^{0}\left(\mathbb{P}^{1}, \mathcal{O}_{\mathbb{P}^{1}}(n)\right)$ mit kanonischer Erweiterung $s \in H^{0}\left(\mathbb{P}^{1}, E^{n}(n)\right)$. Das Bild von $s$ unter der injektiven Abbildung $\operatorname{pr}_{l}: H^{0}\left(\mathbb{P}^{1}, E^{n}(n)\right) \rightarrow H^{0}\left(\mathbb{P}^{1}, E^{l}(n)\right)$ nennen wir kanonische Erweiterung von $\widehat{s_{0}}$ zur Ordnung $l$.

Sei $b_{l, n}(j):=\operatorname{pr}_{l}\left(b_{n, n}(j)\right)$.

Bemerkung 4.16. Für $0 \leq j \leq n$ hat die kanonische Erweiterung $b_{l, n}(j)$ von $\zeta^{j} \in H^{0}\left(\mathbb{P}^{1}, \mathcal{O}_{\mathbb{P}^{1}}(n)\right)$ zur Ordnung l die Gestalt

$$
b_{l, n}(j)=\left(\begin{array}{c}
\zeta^{j} \\
\vdots \\
f_{l, n}(j) \zeta^{j-l}
\end{array}\right) \in H^{0}\left(\mathbb{P}^{1}, E^{l}(n)\right) .
$$

Allgemein können wir den Vektorraum aller globalen holomorphen Schnitte von $E^{l}(n)$ folgendermaßen beschreiben:

Satz 4.17. Seien $l, n$ natürliche Zahlen mit $l \leq n$. Dann gilt:

$$
H^{0}\left(\mathbb{P}^{1}, E^{l}(n)\right) \cong \begin{cases}\bigoplus_{i=0}^{l} H^{0}\left(\mathbb{P}^{1}, \mathcal{O}_{\mathbb{P}^{1}}(n-2 i)\right) & \text { für } l \leq \frac{n+1}{2} \\ H^{0}\left(\mathbb{P}^{1}, \mathcal{O}_{\mathbb{P}^{1}}(n)\right) \oplus \bigoplus_{i=1}^{n-l} H^{0}\left(\mathbb{P}^{1}, \mathcal{O}_{\mathbb{P}^{1}}(n-2 i)\right)^{\vee} & \text { für } l>\frac{n+1}{2} .\end{cases}
$$

Beweis: Für die Dimension von $H^{0}\left(\mathbb{P}^{1}, \mathcal{O}(n-2 l)\right)$ gilt

$$
h^{0}\left(\mathbb{P}^{1}, \mathcal{O}_{\mathbb{P}^{1}}(n-2 l)\right)= \begin{cases}n-2 l+1 & \text { falls } n \geq 2 l \\ 0 & \text { falls } n \leq 2 l-1 .\end{cases}
$$

Außerdem gilt

$$
\begin{aligned}
h^{1}\left(\mathbb{P}^{1}, \mathcal{O}_{\mathbb{P}^{1}}(n-2 l)\right) & =h^{0}\left(\mathbb{P}^{1}, \mathcal{O}_{\mathbb{P}^{1}}(2 l-n-2)\right) \\
& = \begin{cases}0 & \text { falls } n \geq 2 l-1 \\
2 l-n-1 & \text { falls } n<2 l-1 .\end{cases}
\end{aligned}
$$


Wir betrachten nun die in (4.3) definierte exakte Sequenz

$$
0 \rightarrow \mathcal{O}_{\mathbb{P}^{1}}(n-2 l) \rightarrow E^{l}(n) \stackrel{\mathrm{pr}_{l-1}}{\rightarrow} E^{l-1}(n) \rightarrow 0 .
$$

Für $l \leq \frac{n+1}{2}$ ist $H^{1}\left(\mathbb{P}^{1}, \mathcal{O}(n-2 l)\right)=0$, und die zugehörige lange exakte Sequenz ist von der Form

$$
0 \rightarrow H^{0}\left(\mathbb{P}^{1}, \mathcal{O}_{\mathbb{P}^{1}}(n-2 l)\right) \rightarrow H^{0}\left(\mathbb{P}^{1}, E^{l}(n)\right) \rightarrow H^{0}\left(\mathbb{P}^{1}, E^{l-1}(n)\right) \rightarrow 0 .
$$

Induktiv folgt damit für $l \leq \frac{n+1}{2}$ die Behauptung.

Für $l>\frac{n+1}{2}$ ist $H^{0}\left(\mathbb{P}^{1}, \mathcal{O}(n-2 l)\right)=0$, und die zugehörige lange exakte Sequenz ist von der Form

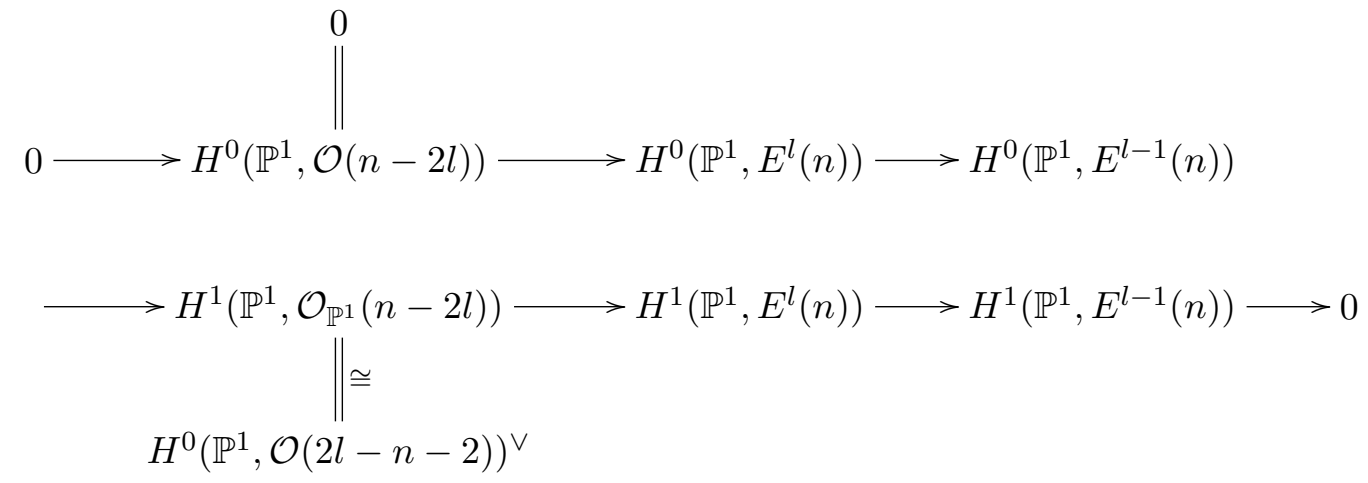

Mit $H^{1}\left(\mathbb{P}^{1}, E^{n}(n)\right)=0$ und $H^{0}\left(\mathbb{P}^{1}, E^{n}(n)\right) \cong H^{0}\left(\mathbb{P}^{1}, \mathcal{O}(n)\right)$ folgt induktiv die Behauptung.

Die kanonischen Erweiterungen von Schnitten aus $H^{0}\left(\mathbb{P}^{1}, \mathcal{O}(n)\right)$ liegen immer in

$$
H^{0}\left(\mathbb{P}^{1}, \mathcal{O}(n)\right) \subset \bigoplus_{i=0}^{l} H^{0}\left(\mathbb{P}^{1}, \mathcal{O}(n-2 i)\right) \cong H^{0}\left(\mathbb{P}^{1}, E^{l}(n)\right)
$$

bzw. in

$$
H^{0}\left(\mathbb{P}^{1}, \mathcal{O}(n)\right) \subset H^{0}\left(\mathbb{P}^{1}, \mathcal{O}(n)\right) \oplus \bigoplus_{i=1}^{n-l} H^{0}\left(\mathbb{P}^{1}, \mathcal{O}(n-2 i)\right)^{\vee} \cong H^{0}\left(\mathbb{P}^{1}, E^{l}(n)\right)
$$

Zusätzlich zur konkreten Berechnung der kanonischen Erweiterung $b_{l, n}(j) \in$ $H^{0}\left(\mathbb{P}^{1}, E^{l}(n)\right)$ eines Schnittes $\zeta^{j} \in H^{0}\left(\mathbb{P}^{1}, \mathcal{O}_{\mathbb{P}^{1}}(n)\right)$ zur Ordnung $l$ können wir auch das Bild von $b_{l, n}(j)$ unter dem Verbindungshomomorphismus $\alpha_{(l)}$ : $H^{0}\left(\mathbb{P}^{1}, E^{l}(n)\right) \rightarrow H^{1}\left(\mathbb{P}^{1}, \mathcal{O}(n-2 l-2)\right)$ genau angeben. 
Definition 4.18. Wir definieren

$$
B_{l, n}(j):=\sum_{c=0}^{l}(-1)^{l-c} \frac{1}{(l-c+1) !} f_{(c, n)}(j) .
$$

Dann gilt

Satz 4.19. Seien $l, n, j \in \mathbb{N}$ mit $l \leq n$ und $j \leq n$. Dann wird das Bild von $b_{l, n}(j) \in H^{0}\left(\mathbb{P}^{1}, E^{l}(n)\right)$ unter dem Verbindungshomomorphismus

$\alpha_{(l)}: H^{0}\left(\mathbb{P}^{1}, E^{l}(n)\right) \rightarrow H^{1}\left(\mathbb{P}^{1}, \mathcal{O}(n-2 l-2)\right)$ repräsentiert durch den Kozykel $\zeta^{j-l-1} B_{l, n}(j)$, wir schreiben

$$
\alpha_{(l)} b_{l, n}(j)=\zeta^{j-l-1} B_{l, n}(j) .
$$

Beweis: $\alpha_{(l)}$ hat nach Definition 4.6 die Form

$$
\left((-1)^{l} \frac{1}{(l+1) !} \zeta^{-(l+1)}, \ldots, \zeta^{-1}\right),
$$

$b_{l, n}(j)$ hat nach (4.6) die Form

$$
\left(\begin{array}{c}
\zeta^{j} \\
\vdots \\
f_{l, n}(j) \zeta^{j-l}
\end{array}\right)
$$

Es folgt

$$
\begin{aligned}
\alpha_{(l)} \cdot b_{l, n}(j) & =\sum_{c=0}^{l}(-1)^{l-c} \frac{1}{(l+1-c) !} \zeta^{-(l+1-c)} f_{(c, n)}(j) \zeta^{j-c} \\
& =\zeta^{j-l-1} B_{l, n}(j) .
\end{aligned}
$$

Bemerkung 4.20. Für $l<n$ ist $\alpha_{(l)} b_{l, n}(j)=0 \in H^{1}\left(\mathbb{P}^{1}, \mathcal{O}(n-2 l-2)\right)$.

Für $l=n$ gilt

$$
B_{n}(j):=B_{n, n}(j)=(-1)^{n-j} \frac{j !(n-j) !}{(n+1) ! n !}
$$

Beweis: Für $l<n$ folgt dies daraus, dass ein Schnitt $b_{l, n}(j) \in H^{0}\left(\mathbb{P}^{1}, E^{l}(n)\right)$ genau dann zu einem Schnitt der Ordnung $l+1$ aus $H^{0}\left(\mathbb{P}^{1}, E^{l+1}(n)\right)$ erweitert 
werden kann, wenn $\alpha_{(l)} b_{l, n}(j)=0 \in H^{1}\left(\mathbb{P}^{1}, \mathcal{O}(n-2(l+1))\right)$ gilt, und daraus, dass $b_{n, n}(j) \in H^{0}\left(\mathbb{P}^{1}, E^{n}(n)\right)$.

Für $l=n$ gilt

$$
\begin{aligned}
B_{n, n}(j) & =\sum_{c=0}^{j}(-1)^{n-c} \frac{1}{(n-c+1) !} \frac{j !(n-c) !}{c !(j-c) ! n !} \\
& =(-1)^{n-j} \frac{(n-j) ! j !}{n !} \sum_{c=0}^{j}(-1)^{j-c}\left(\begin{array}{c}
n-c \\
j-c
\end{array}\right) \frac{1}{c !(n-c+1) !} \\
& =(-1)^{n-j} \frac{j !(n-j) !}{n !(n+1) !} \sum_{c=0}^{j}(-1)^{j-c}\left(\begin{array}{c}
n-c \\
j-c
\end{array}\right)\left(\begin{array}{c}
n+1 \\
c
\end{array}\right) \\
& =(-1)^{n-j} \frac{j !(n-j) !}{n !(n+1) !} \sum_{c=0}^{j}\left(\begin{array}{c}
j-n-1 \\
j-c
\end{array}\right)\left(\begin{array}{c}
n+1 \\
c
\end{array}\right) \\
& =(-1)^{n-j} \frac{j !(n-j) !}{n !(n+1) !} .
\end{aligned}
$$

\section{8 $\quad E^{l}(n)$ und spezielle Fortsetzungen}

Ab sofort betrachten wir wieder Vektorbündel und ihre Schnitte über der Spektralkurve $S$ mit Projektion $\pi: S \rightarrow \mathbb{P}^{1}$. In Definition 4.4 haben wir eine spezielle Fortsetzung eines Schnittes $\widehat{s_{0}} \in H^{0}\left(\mathbb{P}^{1}, \mathcal{O}(m)\right) \subset H^{0}(S, \mathcal{O}(m))$ zur Ordnung $l$ definiert. Als globaler holomorpher Schnitt des Vektorbündels $F^{l}(m)$ wird sie repräsentiert durch

$$
s=\left(\begin{array}{c}
s_{0} \\
\eta^{2} s_{1} \\
\vdots \\
\eta^{2 l} s_{l}
\end{array}\right)
$$

über $U$, wobei $s_{0}$ und das zugehörige $s_{0}^{\prime}$ den Schnitt $\widehat{s_{0}}$ repräsentieren und die einzelnen $s_{i}$ bzw. $s_{i}^{\prime}$ unabhängig von $\eta$ bzw. $\eta^{\prime}$ sind.

Diese speziellen Erweiterungen hängen eng mit den kanonischen Erweiterungen aus $H^{0}\left(\mathbb{P}^{1}, E^{l}(m)\right)$ von Schnitten $\widehat{s_{0}} \in H^{0}\left(\mathbb{P}^{1}, \mathcal{O}_{\mathbb{P}^{1}}(m)\right)$ zusammen.

Satz 4.21. Seien $l, n \in \mathbb{N}$. Dann ist die Menge aller speziellen Fortsetzungen zur Ordnung $l$ aller Elemente aus $\pi^{*} H^{0}\left(\mathbb{P}^{1}, \mathcal{O}(n)\right)$ gleich der Menge aller Schnitte aus $\pi^{*} H^{0}\left(\mathbb{P}^{1}, E^{l}(n)\right)$. 
Beweis: Bei einer speziellen Erweiterung hängen die Funktionen $s_{i} \in \mathcal{O}(U)$ und $s_{i}^{\prime} \in \mathcal{O}\left(U^{\prime}\right)$ nicht mehr von $\eta$ bzw. $\eta^{\prime}$ ab und können deswegen als Funktionen aus $\mathcal{O}\left(U_{0}\right)$ bzw. $\mathcal{O}\left(U_{1}\right)$ aufgefasst werden. $s_{0}, s_{0}^{\prime}$ definieren hierbei einen globalen holomorphen Schnitt aus $\pi^{*} H^{0}\left(\mathbb{P}^{1}, \mathcal{O}(n)\right)$, und allgemein erfüllen die $s_{i}$ bzw. $s_{i}^{\prime}$ einer speziellen Fortsetzung die Bedingung

$$
\begin{aligned}
\eta^{2 i} s_{i} & =\sum_{j=0}^{i} \eta^{2(i-j)} \zeta^{n-i+j} \frac{1}{(i-j) !}\left(\eta^{\prime}\right)^{2 j} s_{j}^{\prime} \\
& =\eta^{2 i} \sum_{j=0}^{i} \frac{1}{(i-j) !} \zeta^{n-i-j} s_{j}^{\prime} .
\end{aligned}
$$

Dies ist genau dann der Fall, wenn gilt

$$
s_{i}=\sum_{j=0}^{i} \frac{1}{(i-j) !} \zeta^{n-i-j} s_{j}^{\prime}=\sum_{j=0}^{i}\left(M^{l}(n)\right)_{i j} s_{j}^{\prime}
$$

mit der Übergangsfunktion $M^{l}(n)$ des Vektorbündels $E^{l}(n)$. Damit folgt die Behauptung.

Folgerung 4.22. Der in Satz 4.21 definierte Isomorphismus zwischen zurückgezogenen Schnitten $\pi^{*} H^{0}\left(\mathbb{P}^{1}, E^{l}(m)\right)$ und der Teilmenge in $H^{0}\left(S, F^{l}(m)\right)$ aller speziellen Fortsetzungen von $\pi^{*} H^{0}\left(\mathbb{P}^{1}, \mathcal{O}_{\mathbb{P}^{1}}(m)\right)$ zur Ordnung l bildet die kanonische Fortsetzung $\pi^{*} b_{l, m}(j)$ von $\zeta^{j} \in \pi^{*} H^{0}\left(\mathbb{P}^{1}, \mathcal{O}_{\mathbb{P}^{1}}(m)\right)$ auf den Schnitt

$$
q_{l, m}(j ; 0):=\left(\begin{array}{c}
\zeta^{j} \\
\eta^{2} f_{1, m}(j) \zeta^{j-1} \\
\vdots \\
\eta^{2 l} f_{l, m}(j) \zeta^{j-l}
\end{array}\right) \in H^{0}\left(S, F^{l}(m)\right)
$$

$a b$.

Damit gilt sogar für beliebiges $n \in \mathbb{N}$

Satz 4.23. Zu jedem Schnitt $p \in \pi_{S}^{*} H^{0}\left(\mathbb{P}^{1}, \mathcal{O}_{\mathbb{P}^{1}}(n)\right)$ gibt es eine eindeutige spezielle Fortsetzung $\bar{p}$ zur Ordnung $n$ zu einem Schnitt von $L^{z}(n)$. Es gibt jedoch keine spezielle Fortsetzung von p zur Ordnung $n+1$ (und damit auch nicht zu höheren Ordnungen).

Definition 4.24. Die zu $s_{0} \in \pi^{*} H^{0}\left(\mathbb{P}^{1}, \mathcal{O}_{\mathbb{P}^{1}}(n)\right)$ eindeutig definierte spezielle Erweiterung der Ordnung $n$ nennen wir ebenfalls die kanonische Erweiterung von $s_{0}$. 


\subsection{Yoneda-Erweiterungen über $S$}

In Abschnitt 4.1 haben wir eine Familie von Geradenbündeln $L^{z}(m)$ über $S$ durch die Übergangsfunktionen $g_{U U^{\prime}}=\zeta^{m} \exp \left(z \frac{\eta^{2}}{\zeta}\right)$ über $U \cap U^{\prime}$ definiert. Hierbei ist $\eta^{2} \zeta^{-1}$ Repräsentant des Elementes $\beta=\eta^{2} \pi^{*} \alpha \in \eta^{2} \pi^{*} H^{1}\left(\mathbb{P}^{1}, \mathcal{O}_{\mathbb{P}^{1}}(-2)\right) \subset$ $H^{1}(S, \mathcal{O})$.

Genauso wie $\alpha$ repräsentiert $\beta \in H^{1}(S, \mathcal{O}) \cong \operatorname{Ext}^{1}(\mathcal{O}, \mathcal{O}) \cong \operatorname{Ext}^{1}(\mathcal{O}(m), \mathcal{O}(m))$ die exakte Sequenz

$$
0 \rightarrow \mathcal{O}_{S}(m) \stackrel{\kappa_{1}}{\rightarrow} F^{1}(m) \stackrel{\mathrm{pr}_{0}}{\rightarrow} \mathcal{O}_{S}(m) \rightarrow 0
$$

von Vektorbündeln über $S$, wobei $F^{1}(m)$ gleich dem in Definition 4.3 definierten Vektorbündel mit Übergangsfunktion

$$
g_{U U^{\prime}}=\widetilde{M^{1}(m)}=\left(\begin{array}{cc}
\zeta^{m} & 0 \\
\eta^{2} \zeta^{m-1} & \zeta^{m}
\end{array}\right)
$$

ist. Hierbei ist $\kappa_{1}$ eine Inklusion und $\mathrm{pr}_{0}$ Projektion.

Da $\beta=\eta^{2} \pi^{*} \alpha$ gilt, erhält man $\beta$ durch Multiplikation von $\pi^{*} \alpha$ aus dem Raum $\operatorname{Ext}^{1}\left(\mathcal{O}_{S}(m), \mathcal{O}_{S}(m-2)\right)$ mit $\eta^{2} \in H^{0}(S, \mathcal{O}(2)) \cong \operatorname{Ext}^{0}\left(\mathcal{O}_{S}(m-2), \mathcal{O}_{S}(m)\right)$ :

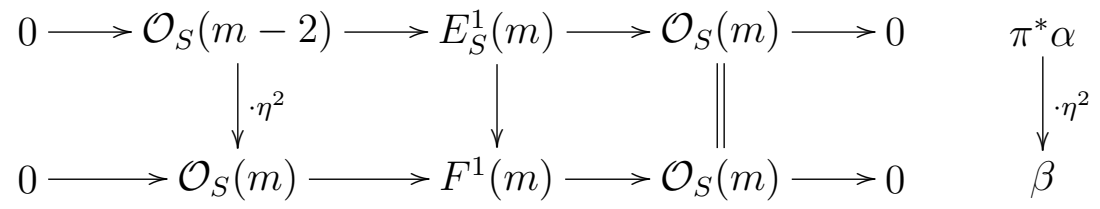

Definition 4.25. Sei $l \in \mathbb{N}$. Dann definieren wir

$$
\beta_{(l)}:=\left((-1)^{l} \frac{1}{(l+1) !} \eta^{2(l+1)} \zeta^{-(l+1)}, \ldots, \eta^{2} \zeta^{-1}\right)
$$

Die in Definition 4.3 gegebene Übergangsfunktion $\widetilde{M^{l}(m)}$ des Vektorbündels $F^{l}(m)$ ist somit von der Form

$$
\widetilde{M^{l}(m)}=\left(\begin{array}{cccc}
\zeta^{m} & 0 & \ldots & 0 \\
\eta^{2} \zeta^{m-1} & \zeta^{m} & \ddots & \vdots \\
\vdots & & \ddots & 0 \\
\frac{1}{l !} \eta^{2 l} \zeta^{m-l} & \ldots & \ldots & \zeta^{m}
\end{array}\right)=\left(\begin{array}{c|c}
\widetilde{M^{l-1}(m)} & \vdots \\
\widetilde{\beta_{(l-1)} \cdot \widetilde{M^{l-1}(m)}} & \zeta^{m}
\end{array}\right)
$$


Dies folgt aus

$$
\begin{aligned}
\left(\beta_{(l-1)} \widetilde{M^{l-1}(m)}\right)_{j} & \left.=\sum_{c=0}^{l-1}(-1)^{l-1-c} \frac{1}{(l-c) !} \eta^{2(l-c)} \zeta^{-(l-c)} \widetilde{\left(M^{l}(m)\right.}\right)_{c j} \\
& =\eta^{2(l-j)} \zeta^{m-l+j} \sum_{c=j}^{l-1}(-1)^{l-1-c} \frac{1}{(l-c) !} \frac{1}{(c-j) !} \\
& =\eta^{2(l-j)} \zeta^{m-l+j} \sum_{c=0}^{l-1-j}(-1)^{l-1-j-c} \frac{1}{(l-c-j) !} \frac{1}{c !} \\
& =\eta^{2(l-j)} \zeta^{m-l+j} \frac{1}{(l-j) !} \underbrace{\sum_{c=0}^{l-j-1}(-1)^{l-1-j-c}\left(\begin{array}{c}
l-j \\
c
\end{array}\right)}_{=\left(-(-1+1)^{l-j}+1\right)} \\
& =\left(\widetilde{M^{l}(m)}\right)_{l, j}
\end{aligned}
$$

mit $0 \leq j \leq l-1$

Wie in Satz 4.7 folgt:

Satz 4.26. Für $l \in \mathbb{N}$ ist $\beta_{(l-1)}$ Repräsentant eines Kozykels aus der Menge $H^{1}\left(\mathbb{P}^{1}, F^{l-1}(m)^{\vee} \otimes \mathcal{O}_{S}(m)\right) \cong \operatorname{Ext}^{1}\left(F^{l-1}(m), \mathcal{O}_{S}(m)\right)$, der die exakte Sequenz von Vektorbündeln

$$
0 \rightarrow \mathcal{O}_{S}(m) \stackrel{\kappa_{l}}{\rightarrow} F^{l}(m) \stackrel{\mathrm{pr}_{l-1}}{\rightarrow} F^{l-1}(m) \rightarrow 0
$$

darstellt. Für die zugehörige lange exakte Sequenz von globalen holomorphen Schnitten

$$
\begin{aligned}
0 & \rightarrow H^{0}(S, \mathcal{O}(m)) \rightarrow H^{0}\left(S, F^{l}(m)\right) \rightarrow H^{0}\left(S, F^{l-1}(m)\right) \\
& \stackrel{\delta}{\rightarrow} H^{1}(S, \mathcal{O}(m)) \rightarrow \ldots
\end{aligned}
$$

ist der Verbindungshomomorphismus $\delta$ gegeben durch Multiplikation mit $\beta_{(l-1)}$. Wir schreiben hierfür $H^{0}\left(S, F^{l-1}(m)\right) \stackrel{\beta_{(l-1)}}{\longrightarrow} H^{1}(S, \mathcal{O}(m))$.

Für die zurückgezogenen Bündel $E_{S}^{l}(m)$ definieren wir

Definition 4.27. Für $l \in \mathbb{N}$ seien induktiv Abbildungen $\varphi_{l}: E_{S}^{l}(m) \rightarrow F^{l}(m)$ folgendermaßen definiert: Sei $\varphi_{1}$ die durch

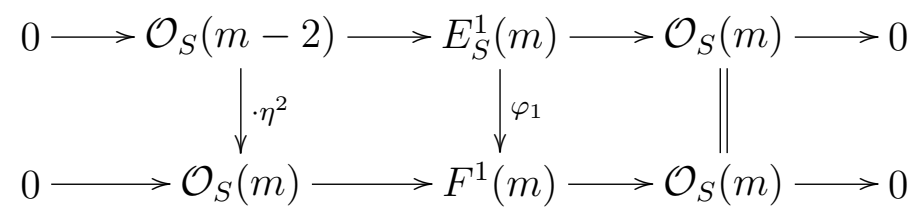


eindeutig definierte Abbildung $\varphi_{1}: E_{S}^{1}(m) \rightarrow F^{1}(m)$. Für $l \geq 1$ sei $\varphi_{l}$ die eindeutig definierte Abbildung $\varphi_{l}: E_{S}^{l}(m) \rightarrow F^{l}(m)$, für die das Diagramm

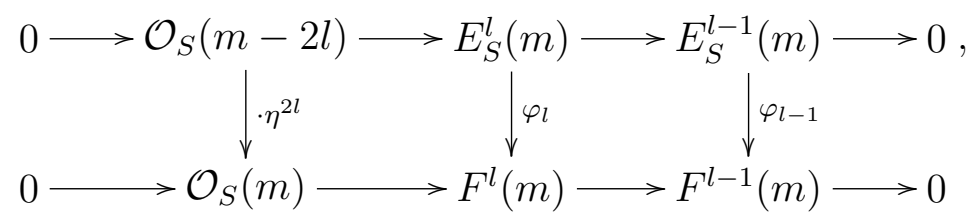

kommutiert.

\subsection{Globale Schnitte von $F^{l}(n)$}

In Satz 2.4 haben wir

$$
H^{0}(S, \mathcal{O}(n))=\bigoplus_{i=0}^{n} \eta^{i} H^{0}\left(\mathbb{P}^{1}, \mathcal{O}(n-i)\right)
$$

gezeigt. Dies können wir verallgemeinern zu

Satz 4.28. Seien $l, n \in \mathbb{N}$. Dann ist

$$
\bigoplus_{i=0}^{n-l} \eta^{i} H^{0}\left(\mathbb{P}^{1}, E^{l}(n-i)\right) \subset H^{0}\left(S, E^{l}(n)\right)
$$

Aufgrund der Eindeutigkeit der Abbildung $\varphi_{l}: H^{0}\left(S, E^{l}(m)\right) \rightarrow H^{0}\left(S, F^{l}(m)\right)$ (Definition 4.27) bildet $\varphi_{l}$ wie in Folgerung 4.22 jede kanonische Fortsetzung $b_{l, m}(j) \in H^{0}\left(\mathbb{P}^{1}, E^{l}(m)\right)$ von $\zeta^{j} \in H^{0}\left(\mathbb{P}^{1}, \mathcal{O}(m)\right) \subset H^{0}(S, \mathcal{O}(m))$ auf $q_{l, m}(j ; 0) \in H^{0}\left(S, F^{l}(m)\right)$ ab.

Definition 4.29. Seien $u, l, n, j \in \mathbb{N}$ mit $j \leq n$ und $u \leq l \leq n$. Sei

$$
q_{l, n}(j ; u):=\left(\begin{array}{c}
0 \\
\vdots \\
0
\end{array}\right\} u
$$

Hierbei steht der Index $n$ für einen zurückgezogenen Schnitt des Bündels $\mathcal{O}_{S}(n), j$ für den $j$-ten Basisvektor $\zeta^{j} \in H^{0}\left(\mathbb{P}^{1}, \mathcal{O}_{\mathbb{P}^{1}}(n)\right) \subset H^{0}\left(S, \mathcal{O}_{S}(n)\right)$, 
$l+1$ für die Anzahl der Einträge von $q_{l, n}(j ; u)$ und $u$ für die Anzahl der ersten $u$ Einträge von $q_{l, n}(j ; u)$, die gleich null sind.

Allgemeiner bildet dann $\varphi_{l}$ jedes $\eta b_{l, m-1}(j) \in \eta H^{0}\left(\mathbb{P}^{1}, E^{l}(m-1)\right)$ auf $q_{l, m-1}(j, 0)$ und, für $0 \leq i \leq m-l$, jedes $\eta^{i} b_{l, m-i}(j) \in \eta^{i} H^{0}\left(\mathbb{P}^{1}, E^{l}(m-i)\right)$ auf $q_{l, m-i}(j ; 0)$ ab.

Geeignete $q_{l, n}(j ; u)$ bilden nun für $l<m$ eine Basis von $H^{0}\left(S, F^{l}(m)\right)$.

Satz 4.30. Sei $l \in \mathbb{N}, l<m$. Dann ist eine Basis von $H^{0}\left(S, F^{l}(m)\right)$ gegeben durch

$$
\begin{gathered}
\left\{q_{l, m-i}(j ; 0) \mid 0 \leq i \leq m-l, 0 \leq j \leq m-i\right\} \\
\bigcup\left\{q_{l, m-i}(j ; 1) \mid 0 \leq i \leq m-l+1,0 \leq j \leq m-i\right\} \\
\vdots \\
\bigcup\left\{q_{l, m-i}(j ; l) \mid 0 \leq i \leq m, 0 \leq j \leq m-i\right\} .
\end{gathered}
$$

Beweis: Jedes $q_{l, m-i}(j ; u)$ mit $0 \leq u \leq l, 0 \leq i \leq m-l+u$ und $0 \leq j \leq m-i$ repräsentiert einen globalen holomorphen Schnitt von $F^{l}(m)$. Dies folgt aus der Tatsache, dass die nichtverschwindenden Einträge

$$
\left(\begin{array}{c}
\eta^{i} \zeta^{j} \\
\vdots \\
\eta^{i+2(l-u)} f_{l-u, m-i}(j) \zeta^{j-l+u}
\end{array}\right)=q_{l-u, m-i}(j ; 0)
$$

von $q_{l, m-i}(j ; u)$ den Schnitt $\varphi_{l-u}\left(\eta^{i} b_{l-u, m-i}(j)\right)$ von $F^{l-u}(m)$ definieren.

Nach Definition sind die $q_{l, m-i}(j ; u)$ linear unabhängig. Induktiv zeigen wir für $l^{\prime}=0, \ldots, l$, dass für $0 \leq u \leq l^{\prime}, 0 \leq i \leq m-l^{\prime}+u$ und $0 \leq j \leq m-i$ die $q_{l^{\prime}, m-i}(j ; u)$ tatsächlich den ganzen Raum $H^{0}\left(S, F^{l^{\prime}}(m)\right)$ aufspannen.

Für $l^{\prime}=0$ ist

$$
H^{0}\left(S, F^{0}(m)\right)=H^{0}(S, \mathcal{O}(m))=\bigoplus_{i=0}^{m} \eta^{i} H^{0}\left(\mathbb{P}^{1}, \mathcal{O}(m-i)\right)
$$

und die $q_{0, m-i}(j ; 0)$ bilden eine Basis von $H^{0}(S, \mathcal{O}(m))$.

$\beta$ bildet $\eta^{m} H^{0}\left(\mathbb{P}^{1}, \mathcal{O}\right)$ isomorph auf $\eta^{m+2} H^{1}\left(\mathbb{P}^{1}, \mathcal{O}(-2)\right) \subset H^{1}(S, \mathcal{O}(m))$ ab, alle anderen Untervektorräume $\eta^{i} H^{0}\left(\mathbb{P}^{1}, \mathcal{O}(m-i)\right)$ von $H^{0}(S, \mathcal{O}(m))$ werden auf null abgebildet. Mit der für $l=1$ zur exakten Sequenz

$$
0 \rightarrow \mathcal{O}_{S}(m) \stackrel{\kappa_{l}}{\rightarrow} F^{l}(m) \stackrel{\mathrm{pr}_{l-1}}{\rightarrow} F^{l-1}(m) \rightarrow 0
$$


aus (4.9) gehörigen langen exakten Sequenz

$$
\begin{aligned}
& 0 \rightarrow H^{0}(S, \mathcal{O}(m)) \stackrel{\kappa_{1}}{\rightarrow} H^{0}\left(S, F^{1}(m)\right) \stackrel{\mathrm{pr}_{0}}{\rightarrow} H^{0}(S, \mathcal{O}(m)) \\
& \quad \stackrel{\beta}{\rightarrow} H^{1}(S, \mathcal{O}(m)) \rightarrow \ldots
\end{aligned}
$$

folgt die Behauptung für $l^{\prime}=1$ : Es ist $\kappa_{1}\left(q_{0, m-i}(j ; 0)\right)=q_{1, m-i}(j ; 1)$ mit $0 \leq i \leq m$ und $0 \leq j \leq m-i$, und $\operatorname{pr}_{0}\left(q_{1, m-i}(j ; 0)\right)=q_{0, m-i}(j ; 0)$ und $\beta\left(q_{0, m-i}(j ; 0)\right)=0$ für $0 \leq i \leq m-1,0 \leq j \leq m-i$.

Für beliebiges $l \leq m$ bildet $\beta_{(l)}$ jeden $\operatorname{Schnitt~} q_{l, m-i}(j ; u) \in H^{0}\left(S, F^{l}(m)\right)$ auf

$$
\begin{aligned}
\beta_{(l)} q_{l, m-i}(j ; u) & =\beta_{(l-u)} q_{l-u, m-i}(j ; 0) \\
& =\sum_{c=0}^{l-u}(-1)^{l-u-c} \frac{\eta^{2(l-u+1-c)} \zeta^{-(l-u+1-c)}}{(l-u+1-c) !} \eta^{i+2 c} f_{c, m-i}(j) \zeta^{j-c} \\
& =\eta^{2 l-2 u+i+2} \zeta^{j-l+u-1} \sum_{c=0}^{l-u}(-1)^{l-u-c} \frac{1}{(l-u+1-c) !} f_{c, m-i}(j) \\
& =\eta^{2 l-2 u+2+i} \zeta^{j-l+u-1} B_{l-u, m-i}(j) \\
& = \begin{cases}\eta^{m+l+2-u} \zeta^{j-l+u-1} \frac{j !(l-u-j) !}{(l-u+1) !(l-u) !} & \text { falls } i=m+u-l \\
0 & \text { sonst }\end{cases}
\end{aligned}
$$

ab. Damit folgt für $0 \leq u \leq l^{\prime}-1$

$$
\beta_{\left(l^{\prime}-1\right)}\left(\left\langle q_{l^{\prime}-1, l^{\prime}-u-1}(j ; u)\right\rangle_{0 \leq j \leq l^{\prime}-u-1}\right)=\eta^{m+l^{\prime}+1-u} H^{1}\left(\mathbb{P}^{1}, \mathcal{O}\left(u-l^{\prime}-1\right)\right),
$$

alle anderen $q_{l^{\prime}-1, m-i}(j ; u)$ werden auf null abgebildet.

Spannen für $l^{\prime}$ mit $0 \leq u \leq l^{\prime}-1,0 \leq i \leq m-l^{\prime}+1+u$ die $q_{l^{\prime}-1, m-i}(j ; u)$ den ganzen Raum $H^{0}\left(S, F^{l^{\prime}-1}(m)\right)$ auf, so folgt aus der langen exakten Sequenz

$$
\begin{aligned}
0 & \rightarrow H^{0}(S, \mathcal{O}(m)) \stackrel{\kappa_{l^{\prime}}}{\rightarrow} H^{0}\left(S, F^{l^{\prime}}(m)\right) \stackrel{\mathrm{pr}_{l^{\prime}-1}}{\rightarrow} H^{0}\left(S, F^{l^{\prime}-1}(m)\right) \\
\stackrel{\beta_{\left(l^{\prime}-1\right)}}{\rightarrow} H^{1}(S, \mathcal{O}(m)) & \rightarrow \ldots
\end{aligned}
$$

$\mathrm{zu}(4.9)$ mit $\kappa_{l^{\prime}}\left(q_{0, m-i}(j ; 0)\right)=q_{l^{\prime}, m-i}\left(j ; l^{\prime}\right)$ und, für $u<l^{\prime}, \operatorname{pr}_{l^{\prime}-1}\left(q_{l^{\prime}, m-i}(j ; u)\right)=$ $q_{l^{\prime}-1, m-i}(j ; u)$ die Behauptung.

Folgerung 4.31. Sei $0 \leq u \leq l<m$. Dann gilt für

$$
Q_{l}(u):=\left\langle q_{l, m-i}(j ; u)\right\rangle_{0 \leq i \leq m-l+u, 0 \leq j \leq m-i},
$$

dass

$$
H^{0}\left(S, F^{l}(m)\right)=\bigoplus_{u=0}^{l} Q_{l}(u) .
$$


Bemerkung 4.32. Auch für $l \geq m$ definieren die Vektoren

$$
\left\{q_{l, m-i}(j ; u) \mid 0 \leq u \leq l, 0 \leq i \leq m-l+u, 0 \leq j \leq m-i\right\}
$$

linear unabhängige globale holomorphe Schnitte von $F^{l}(m)$. Es gilt

$$
\bigoplus_{u=0}^{l} Q_{l}(u) \subsetneq H^{0}\left(S, F^{l}(m)\right) .
$$

Die Abbildung $\beta_{(l)}: H^{0}\left(S, F^{l}(m)\right) \rightarrow H^{1}(S, \mathcal{O}(m))$ bildet $q_{l, m-i}(j ; u)$ auf

$$
\begin{aligned}
\beta_{(l)} q_{l, m-i}(j ; u) & =\eta^{2 l-2 u+2+i} \zeta^{j-l+u-1} B_{l-u, m-i}(j) \\
& = \begin{cases}\eta^{m+l+2-u} \zeta^{j-l+u-1} B_{m-i, m-i}(j) & \text { falls } i=m+u-l \\
0 & \text { sonst }\end{cases}
\end{aligned}
$$

ab. Für die kanonischen Fortsetzungen $b_{l-u, m-i}(j)$ von $\zeta^{j} \in \pi^{*} H^{0}\left(\mathbb{P}^{1}, \mathcal{O}(m-i)\right)$ folgt

$$
\begin{aligned}
\beta_{(l)} q_{l, m-i}(j ; u) & =\eta^{2 l-2 u+2+i} \zeta^{j-l+u-1} B_{l-u, m-i}(j) \\
& =\eta^{2 l-2 u+2+i} \alpha_{(l-u)} b_{l-u, m-i}(j) .
\end{aligned}
$$

\subsection{Genaue Beschreibung von $V_{0}$}

Wir wollen nun Erweiterungen von globalen holomorphen Schnitten $s_{0}$ von $\mathcal{O}_{S}(m)$ zur Ordnung $l$, d.h. Schnitte $s$ aus $H^{0}\left(S, F^{l}(m)\right)$ mit $\operatorname{pr}_{0}(s)=s_{0}$, berechnen.

Der Hinderungsgrund dafür, dass eine Erweiterung $s$ zur Ordnung $l$ nicht zu höheren Ordnungen erweitert werden kann, liegt immer im Raum

$$
H^{1}(S, \mathcal{O}(m))=\bigoplus_{i=2}^{m} \eta^{m+i} H^{1}\left(\mathbb{P}^{1}, \mathcal{O}(-i)\right)
$$

Ist $l<m$ und $\eta^{i} \zeta^{j} \in \eta^{i} H^{0}\left(\mathbb{P}^{1}, \mathcal{O}(m-i)\right) \subset H^{0}(S, \mathcal{O}(m))$ mit $0 \leq j \leq m-i$, so ist für $0 \leq i \leq m-l$ die Menge aller Erweiterungen von $\eta^{i} \zeta^{j}$ zur Ordnung $l$ gegeben durch Elemente

$$
q_{l, m-i}(j ; 0)+\sum_{\substack{1 \leq u \leq l \\ 0 \leq g \leq m \leq l+u \\ 0 \leq d \leq m-g}} c_{u g d} q_{l, m-g}(d ; u)
$$

mit komplexen Zahlen $c_{\text {ugd }} \in \mathbb{C}$. 
Bemerkung 4.33. Aus Satz 4.30 folgt, dass es für $i>m-l$ keine Erweiterungen von Elementen aus $\eta^{i} H^{0}\left(\mathbb{P}^{1}, \mathcal{O}(m-i)\right.$ ) zur Ordnung $l$ (und damit auch keine höheren Erweiterungen) gibt.

Beispiel 4.34. Sei $\eta^{m-1} \zeta$ ein Basisvektor von $\eta^{m-1} H^{0}\left(\mathbb{P}^{1}, \mathcal{O}(1)\right) \subset H^{0}(S, \mathcal{O}(m))$ mit kanonischer Erweiterung $\varphi_{1}\left(\eta^{m-1} b_{1,1}(1)\right)=q_{1,1}(1 ; 0) \in H^{0}\left(S, F^{1}(m)\right)$. Dann ist $\beta_{(1)} q_{1,1}(1 ; 0)$ ungleich null und wird repräsentiert durch das Element $\frac{1}{2} \eta^{m+3} \zeta^{-1}$ aus $\eta^{m+3} H^{1}\left(\mathbb{P}^{1}, \mathcal{O}(-3)\right)$. Damit gibt es keinen globalen holomorphen Schnitt aus $H^{0}\left(S, F^{2}(m)\right)$, dessen Bild unter $\operatorname{pr}_{1}$ gleich $q_{1,1}(1,0)$ ist, da $\eta^{m-1} \zeta$ nicht im Kern der Abbildung $\beta_{(1)}$ liegt. Die Menge aller Erweiterungen von $\eta^{m-1} \zeta$ zur Ordnung 1 ist gegeben durch

$$
q_{1 ; 1}(1,0)+\sum_{\substack{0 \leq i \leq m \\ 0 \leq j \leq m-i}} c_{i j} q_{1, m-i}(j ; 1) .
$$

Da jedoch

$$
\beta_{(1)} q_{1, m-i}(j ; 1)= \begin{cases}\eta^{m+2} \zeta^{-1} & \text { falls } i=m \\ 0 & \text { sonst }\end{cases}
$$

ist, gibt es zu keiner Erweiterung $s \in H^{0}\left(S, F^{1}(m)\right)$ von $\eta^{m-1} \zeta$ ein Element aus $H^{0}\left(S, F^{2}(m)\right)$, dessen Bild unter $\operatorname{pr}_{1}$ gleich $s$ ist.

Kommen wir nun zum Hauptresultat dieses Abschnittes. Die Faser $V_{0}$ des in Abschnitt 4.1 definierten Vektorbündels $V$ besteht aus den Schnitten aus $H^{0}(S, \mathcal{O}(m))$, die sich zu Schnitten von $H^{0}\left(S, L^{z}(m)\right)$ mit $|z|$ hinreichend klein fortsetzen lassen, siehe hierzu Abschnitt 4.2. Es gilt

Satz 4.35. $V_{0}=H^{0}\left(\mathbb{P}^{1}, \mathcal{O}(m)\right) \oplus \eta H^{0}\left(\mathbb{P}^{1}, \mathcal{O}(m-1)\right)$.

Beweis: Das Vektorbündel $V$ hat den Rang $k$. Da die Dimension des Raumes $H^{0}\left(\mathbb{P}^{1}, \mathcal{O}(m)\right) \oplus \eta H^{0}\left(\mathbb{P}^{1}, \mathcal{O}(m-1)\right)$ gleich $2 m+1=k$ ist, genügt es, $V_{0} \subset H^{0}\left(\mathbb{P}^{1}, \mathcal{O}(m)\right) \oplus \eta H^{0}\left(\mathbb{P}^{1}, \mathcal{O}(m-1)\right)$ zu zeigen.

$V_{0}$ ist eine Teilmenge von

$$
H^{0}(S, \mathcal{O}(m))=\bigoplus_{i=0}^{m} \eta^{i} H^{0}\left(\mathbb{P}^{1}, \mathcal{O}(m-i)\right)
$$

Wir haben in Bemerkung 4.33, folgend aus Satz 4.30, gesehen, dass für $i>1$ die Schnitte aus $\eta^{i} H^{0}\left(\mathbb{P}^{1}, \mathcal{O}(m-i)\right)$ keine Fortsetzungen zur Ordnung $m-1$ oder höherer Ordnung besitzen. Damit folgt

$$
V_{0} \subset H^{0}\left(\mathbb{P}^{1}, \mathcal{O}(m)\right) \oplus \eta H^{0}\left(\mathbb{P}^{1}, \mathcal{O}(m-1)\right)
$$

und damit die Behauptung. 


\subsection{Konkrete Erweiterungen}

Der Beweis von Satz 4.35 ist ein Existenzbeweis. Fortsetzungen von Elementen aus $H^{0}\left(\mathbb{P}^{1}, \mathcal{O}(m)\right) \oplus \eta H^{0}\left(\mathbb{P}^{1}, \mathcal{O}(m-1)\right)$ zur Ordnung $l$ für beliebiges $l \in \mathbb{N}$ kann man auch konkret berechnen. Insbesondere können für $u \in \mathbb{N}$ die ersten $u$ Einträge für Fortsetzungen beliebiger Ordnungen berechnet werden.

\section{Fortsetzungen von Elementen aus $\eta H^{0}\left(\mathbb{P}^{1}, \mathcal{O}(m-1)\right)$}

Sei $0 \leq j \leq m-1$. Wir bestimmen im Folgenden für die Basis $\eta \zeta^{j}$ von $\eta H^{0}\left(\mathbb{P}^{1}, \mathcal{O}(m-1)\right)$ konkret die ersten Einträge von Fortsetzungen beliebiger Ordnung.

Aus Satz 4.30 folgt

Satz 4.36. Sei $l<m$. Dann ist die Menge aller Erweiterungen von $\eta \zeta^{j} z u r$ Ordnung l gegeben durch

$$
\begin{aligned}
\mathcal{V}(l):=\left\{q_{l, m-1}(j ; 0)\right. & +\sum c_{u g d} q_{l, m-g}(d ; u) \\
& \left.\mid 1 \leq u \leq l, 0 \leq g \leq m-l+u, 0 \leq d \leq m-g, c_{u g d} \in \mathbb{C}\right\} .
\end{aligned}
$$

Aufgrund von Satz 4.26 wissen wir, dass es zu einem Element $v \in \mathcal{V}(l)$ genau dann eine Erweiterung zur Ordnung $l+1$ gibt, wenn gilt $\beta_{(l)} v=0 \in$ $H^{1}(S, \mathcal{O}(m))$.

Mit Bemerkung 4.32,

$$
\beta_{(l)} q_{l, m-g}(d ; u)= \begin{cases}\eta^{m+l+2-u} \zeta^{d-l+u-1} B_{m-g}(d) & \text { falls } g=m+u-l \\ 0 & \text { sonst }\end{cases}
$$

folgt $\beta_{(l)} q_{l, m-1}(j ; 0)=0$ für $l<m-1$. Für $l=m-1$ gilt

Satz 4.37. Die Basisvektoren $q_{m-1, m-1-u}(d ; u)$ von $H^{0}\left(S, F^{m-1}(m)\right)$ mit $0 \leq$ $u \leq m-1$ und $0 \leq d \leq m-1-u$ werden unter der Abbildung $\beta_{(m-1)}$ : $H^{0}\left(S, F^{m-1}(m)\right) \rightarrow H^{1}(S, \mathcal{O}(m))$ auf

$$
\beta_{(m-1)} q_{m-1, m-1-u}(d ; u)=\eta^{2 m+1-u} \zeta^{d-m+u} B_{m-1-u}(d)
$$

ungleich null abgebildet.

Trotzdem gibt es einen Unterraum von $\left\langle q_{m-1, m-1-u}(d ; u)\right| 0 \leq u \leq m-1,0 \leq$ $d \leq m-u-1\rangle$, der im Kern der Abbildung $\beta_{(m-1)}$ liegt. Ergänzend zu Definition 4.29 sei $q_{l, n}(j ; u)=0$, falls $j \in \mathbb{Z}$ negativ oder $j>n$ ist. Dann gilt: 
Satz 4.38. Nur Linearkombinationen von Elementen

$$
\begin{aligned}
& v_{m-1}(j):=q_{m-1, m-1}(j ; 0)+ \\
& \sum_{i=2}^{m}\left(\sum_{c=0}^{m+1-i} a_{m+1-i}(c) \frac{B_{m-1}(j) q_{m-1, i-2}(j-m+c+i-1, m+1-i)}{B_{i-2}(j-m+c+i-1)}\right)
\end{aligned}
$$

mit $0 \leq j \leq m-1$ liegen im Kern von $\beta_{(m-1)}$ eingeschränkt auf den Raum $\left\langle q_{m-1, m-1-u}(j ; u) \mid 0 \leq u \leq m-1,0 \leq j \leq m-u-1\right\rangle$.

Beweis: Für festes $1 \leq u \leq m-1$ sind die Bilder von $q_{m-1, m-1-u}(d ; u)$ mit $0 \leq d \leq m-1-u$ unter $\beta_{(m-1)}$ linear unabhängig in $\eta^{2 m+1-u} H^{1}\left(\mathbb{P}^{1}, \mathcal{O}(-m+\right.$ $u-1)) \subset H^{1}(S, \mathcal{O}(m))$, d.h. die Elemente

$$
\begin{aligned}
& \left\{\beta_{(m-1)} q_{m-1, m-1-u}(d ; u) \mid 1 \leq u \leq m-1,0 \leq d \leq m-1-u\right\} \\
& =\left\{\beta_{(m-1)} q_{m-1, i-2}(d ; m+1-i) \mid 2 \leq i \leq m, 0 \leq d \leq i-2\right\}
\end{aligned}
$$

bilden eine Basis von $H^{1}(S, \mathcal{O}(m))$. Wir stellen $\beta_{(m-1)} q_{m-1, m-1}(j ; 0)$ bezüglich dieser Basis dar.

Über $S$ gilt nach Definition der Spektralkurve $S$

$$
\eta^{2 m+1}=-a_{1} \eta^{2 m}-\ldots-a_{k}
$$

mit $a_{i} \in H^{0}\left(\mathbb{P}^{1}, \mathcal{O}(i)\right) . a_{i}$ wird hierbei durch ein Polynom $a_{i}(0)+\zeta a_{i}(1)+\ldots+$ $\zeta^{i} a_{i}(i)$ vom Grad $i$ dargestellt. Damit folgt

$$
\begin{aligned}
\beta_{(m-1)} q_{m-1, m-1}(j ; 0)= & \eta^{2 m+1} \zeta^{j-m} B_{m-1}(j) \\
= & \sum_{c=0}^{2 m} \eta^{c}\left(-a_{2 m+1-c} \zeta^{j-m}\right) B_{m-1}(j) \\
= & \eta^{2 m}\left(-a_{1} \zeta^{j-m} B_{m-1}(j)\right)+\eta^{2 m-1}\left(-a_{2} \zeta^{j-m} B_{m-1}(j)\right)+\ldots \\
& \quad+\eta^{m+2}\left(-a_{m-1} \zeta^{j-m} B_{m-1}(j)\right) \\
& \quad+\left(\eta^{m+1}\left(-a_{m} \zeta^{j-m} B_{m-1}(j)\right)+\ldots+\left(-a_{k} \zeta^{j-m} B_{m-1}(j)\right)\right) .
\end{aligned}
$$

Folglich wird $\beta_{(m-1)} q_{m-1, m-1}(j ; 0) \in H^{1}(S, \mathcal{O}(m))$ repräsentiert durch

$$
\beta_{(m-1)} q_{m-1, m-1}(j ; 0)=\sum_{i=2}^{m} \eta^{m+i}\left[-a_{m+1-i} \zeta^{j-m}\right] B_{m-1}(j) .
$$

Die eckigen Klammern $\left[-a_{m+1-i} \zeta^{j-m}\right]$ bezeichnen hierbei den Repräsentanten von $-a_{m+1-i} \zeta^{j-m}$ in $H^{1}\left(\mathbb{P}^{1}, \mathcal{O}(-i)\right)$, d.h.

$$
\left[-a_{m+1-i} \zeta^{j-m}\right]=\sum_{\substack{c \in \mathbb{N} \text { mit } \\-i+1 \leq j-m+c \leq-1}}\left(-a_{m+1-i}(c)\right) \zeta^{j-m+c} .
$$


Mit

$$
\begin{aligned}
& \beta_{(m-1)}\left(a_{m+1-i}(c) \frac{B_{m-1}(j) q_{m-1, i-2}(j-m+c+i-1, m+1-i)}{B_{i-2}(j-m+c+i-1)}\right) \\
& = \begin{cases}a_{m+1-c}(c) B_{m-1}(j) \eta^{m+i} \zeta^{j-m+c} & \text { falls }-i+1 \leq j-m+c \leq-1 \\
0 & \text { sonst }\end{cases}
\end{aligned}
$$

folgt die Behauptung.

Zu diesen Elementen $v_{m-1}(j) \in H^{0}\left(S, F^{m-1}(m)\right)$ gibt es folglich Fortsetzungen $v_{m}(j) \in H^{0}\left(S, F^{m}(m)\right)$ mit $\operatorname{pr}_{m-1}\left(v_{m}(j)\right)=v_{m-1}(j)$. Es gilt:

Satz 4.39. Der Vektor

$$
v_{m}(j):=\left(\begin{array}{c}
v_{m-1}(j) \\
s_{m}(j)
\end{array}\right)
$$

mit

$$
s_{m}(j):=\sum_{c=0}^{2 m} \eta^{c}\left(-a_{2 m+1-c} \zeta^{j-m}\right)^{+} B_{m-1}(j)
$$

repräsentiert einen Schnitt aus $H^{0}\left(S, F^{m}(m)\right)$ mit $\operatorname{pr}_{m}\left(v_{m}\right)=v_{m-1}$. Hierbei bezeichne $\left(-a_{2 m+1-c} \zeta^{j-m}\right)^{+}$den polynomialen Anteil von $-a_{2 m+1-c} \zeta^{j-m}$, d.h. die Summe $\sum_{\substack{d \in \mathbb{N} m i t \\ m-j \leq d \leq 2 m+1-c}}\left(-a_{2 m+1-c}(d) \zeta^{j-m+d}\right)$.

Beweis: Da für $0 \leq j \leq m-1$ jedes $v_{m-1}(j) \in H^{0}\left(S, F^{m-1}(m)\right)$ nach Satz $4.38 \mathrm{im}$ Kern von $\beta_{(m-1)}$ liegt, gibt es eine Fortsetzung von $v_{m-1}(j) \mathrm{zu}$ einem Schnitt von $H^{0}\left(S, F^{m}(m)\right)$. Somit gibt es Abbildungen $s \in \mathcal{O}(U)$ und $s^{\prime} \in \mathcal{O}\left(U^{\prime}\right)$, so dass über $U \cap U^{\prime}$

$$
\left(\begin{array}{c|c}
\widetilde{M^{m-1}(m)} & 0 \\
& 0 \\
\hline \beta_{(m-1)} \cdot \widetilde{M^{m-1}(m)} & \zeta^{m}
\end{array}\right) \cdot\left(\begin{array}{c}
v_{m-1}^{\prime}(j) \\
s^{\prime}
\end{array}\right)=\left(\begin{array}{c}
v_{m-1}(j) \\
\hline s
\end{array}\right)
$$

gilt.

Da $\widetilde{M^{m-1}(m)} v_{m-1}^{\prime}(j)=v_{m-1}(j)$ ist, müssen die holomorphen Funktionen $s=\sum_{g, h \geq 0} d_{g h} \eta^{g} \zeta^{h}$ und $s^{\prime}=\sum_{g, h \geq 0} d_{g h}^{\prime}\left(\eta^{\prime}\right)^{g}\left(\zeta^{\prime}\right)^{h}$ mit komplexen Zahlen $d_{g h}$ und 
$d_{g h}^{\prime}$ die Bedingung

$$
\beta_{(m-1)} v_{m-1}(j)+\zeta^{m} \sum_{g, h \geq 0} d_{g h}^{\prime} \eta^{g} \zeta^{-g-h}=\sum_{g, h \geq 0} d_{g h} \eta^{g} \zeta^{h}
$$

über $U \cap U^{\prime}$ erfüllen. Sei $s:=s_{m}(j)$ und

$$
s^{\prime}:=s_{m}^{\prime}(j):=\sum_{c=0}^{2 m} \eta^{c}\left(a_{2 m+1-c} \zeta^{j-m}\right)^{-} B_{m-1}(j) .
$$

Hierbei sei $\left(a_{2 m+1-c} \zeta^{j-m}\right)^{-}$definiert als

$$
\sum_{\substack{d \in \mathbb{N} \operatorname{mit} \\ j-m+d<\min \{0, m-c+1\}}} a_{2 m+1-c}(d) \zeta^{j-m+d} .
$$

Mit $\beta_{(m-1)}\left(q_{m-1, m-1}(j ; 0)\right)=\sum_{c=0}^{2 m} \eta^{c}\left(-a_{2 m+1-c} \zeta^{j-m}\right) B_{m-1}(j)$ und der Definition von $v_{m-1}(j)$ folgt die Behauptung.

Insgesamt folgt:

Bemerkung 4.40. Eine Basis von $H^{0}\left(S, F^{m}(m)\right)$ ist gegeben durch

$$
\begin{aligned}
& \left\{q_{m, m}(j ; 0) \mid 0 \leq j \leq m\right\} \\
\cup & \left\{q_{m, m-i}(j ; 1) \mid 0 \leq i \leq 1,0 \leq j \leq m-i\right\} \\
\vdots & \\
\cup & \left\{q_{m, m-i}(j ; m) \mid 0 \leq i \leq m, 0 \leq j \leq m-i\right\} \\
\cup & \left\{v_{m}(j) \mid 0 \leq j \leq m-1\right\} .
\end{aligned}
$$

Die Menge aller Fortsetzungen von $\eta \zeta^{j}$ zur Ordnung $m$ ist somit gegeben durch

$\left\{v_{m}(j)+\sum c_{\text {ugd }} q_{m, m-g}(d ; u) \mid 1 \leq u \leq m, 0 \leq g \leq u, 0 \leq d \leq m-g, c_{\text {ugd }} \in \mathbb{C}\right\}$.

\section{Allgemeine Fortsetzungen}

Alle Bilder unter der Abbildung $\beta_{(l)}$ von Elementen $v$ aus $H^{0}\left(S, F^{l}(m)\right), l \in \mathbb{N}$, liegen in $H^{1}(S, \mathcal{O}(m))$ und können nach Satz 2.6 dargestellt werden als

$$
\beta_{(l)}(v)=\sum_{i=2}^{m} \eta^{m+i} \pi^{*} h_{i}
$$


mit passenden $h_{i} \in H^{1}\left(\mathbb{P}^{1}, \mathcal{O}(-i)\right)$. Diese Darstellung nutzen wir in allen Berechnungen. Wird ein Element aus $H^{1}(S, \mathcal{O}(m))$ durch eine höhere Potenz $\eta^{2 m+i} \cdot($ Konstante) dargestellt, nutzen wir induktiv die Bedingung

$$
\eta^{2 m+1}+a_{1} \eta^{2 m}+\ldots+a_{k}=0
$$

über $S$ mit $a_{i} \in H^{0}\left(\mathbb{P}^{1}, \mathcal{O}(i)\right)$, um diesen Term in der Form (4.11) darzustellen. Allgemein definieren wir:

Definition 4.41. Für $i, j \in \mathbb{N}$ mit $j \leq k$ seien Polynome $P_{i, j} \in H^{0}\left(\mathbb{P}^{1}, \mathcal{O}(j+\right.$ $i-1)$ ) induktiv definiert durch

$$
P_{1, j}:=-a_{j}
$$

und

$$
\eta^{2 m+i}=P_{i, 1} \eta^{k-1}+P_{i, 2} \eta^{k-2}+\ldots+P_{i, k}
$$

Dann ist

$$
P_{i+1, j}= \begin{cases}P_{1, j} P_{i, 1}+P_{i, j+1} & \text { falls } j<k \\ P_{1 k} P_{i 1} & \text { falls } j=k\end{cases}
$$

Die $h_{i} \in H^{1}\left(\mathbb{P}^{1}, \mathcal{O}(-i)\right)$ erhalten wir aus

Satz 4.42. Sei $l \geq m-1$. Die Elemente $\beta_{(l)} q_{l, l-u}(j ; u)$ in $H^{1}(S, \mathcal{O}(m))$ mit $l-m+2 \leq u \leq l$ und $0 \leq j \leq l-u$ bilden eine Basis von $H^{1}(S, \mathcal{O}(m))$.

Beweis: Dies folgt aus

$$
\begin{aligned}
\beta_{(l)} q_{l, l-u}(j ; u) & =\eta^{m+(l-u+2)} \zeta^{j-l+u-1} B_{l-u}(j) \\
& \in \eta^{m+(l-u+2)} H^{1}\left(\mathbb{P}^{1}, \mathcal{O}(u-l-2)\right) .
\end{aligned}
$$

Zur konkreten Berechnung einer Erweiterung eines Elementes $\zeta^{j} \in H^{0}\left(\mathbb{P}^{1}, \mathcal{O}(m)\right)$ mit $0 \leq j \leq m$ bzw. $\eta \zeta^{j} \in H^{0}\left(\mathbb{P}^{1}, \mathcal{O}(m-1)\right)$ mit $0 \leq j \leq m-1$ haben wir den folgenden Algorithmus entwickelt:

1. Seien $w_{l}(j)$ bzw. $v_{l}(j) \in H^{0}\left(S, F^{l}(m)\right)$ jeweils eine Erweiterung von $\zeta^{j}$ bzw. $\eta \zeta^{j}$ zur Ordnung $l$. Ohne Einschränkung gelte $l \geq m-1$. Wir berechnen jeweils das Polynom $\beta_{(l)} w_{l}(j)$ bzw. $\beta_{(l)} v_{l}(j)$.

2. Höhere Potenzen $\eta^{2 m+i}$ mit $i \geq 1$ in den Polynomen $\beta_{(l)} w_{l}(j)$ bzw. $\beta_{(l)} v_{l}(j)$ ersetzen wir durch die in Definition 4.41 gegebenen Polynome $P_{i 1} \eta^{k-1}+\ldots+P_{i k}$. 
3. Wir stellen das durch den Kozyklus $\beta_{(l)} w_{l}(j)$ bzw. $\beta_{(l)} v_{l}(j)$ gegebene Element aus $H^{1}(S, \mathcal{O}(m))$ als Linearkombination der Basis $\beta_{(l)} q_{l, l-u}(j ; u)$ aus Satz 4.42 dar.

4. Dadurch erhalten wir Korrekturterme zu $w_{l}(j)$ bzw. $v_{l}(j)$ als Urbilder der in 3. berechneten Linearkombinationen. Da gilt $l-m+2 \leq u \leq l$ lassen diese die ersten $l-m+2$ Einträge der $w_{l}(j)$ bzw. $v_{l}(j)$ unverändert.

5. Schließlich konstruieren wir Abbildungen $s_{l+1}: U \rightarrow \mathbb{C}$ und $s_{l+1}^{\prime}: U^{\prime} \rightarrow \mathbb{C}$, so dass $\left(v_{l}(j)^{t}, s_{l+1}\right)^{t}$ einen Schnitt aus $H^{0}\left(S, F^{l+1}(m)\right)$ definiert. Durch die Bedingung $\beta_{(l)} v_{l}(j)+\zeta^{m} s^{\prime}\left(\eta^{\prime}, \zeta^{\prime}\right)=s(\eta, \zeta)$ an $s, s^{\prime}$ über $U \cap U^{\prime}$ können wir $s$ und $s^{\prime}$ mithilfe des in 2. bestimmten Polynoms angeben: $s$ ist sein polynomialer Anteil, $s^{\prime}$ der Hauptteil ohne die Terme, die Kozykeln aus $H^{1}\left(\mathbb{P}^{1}, \mathcal{O}(-i)\right)$ mit $2 \leq i \leq m$ definieren.

Zum Beispiel gilt

$$
\begin{aligned}
\beta_{(m)} q_{m, m}(j ; 0)= & \eta^{2 m+2} \zeta^{j-m-1} B_{m}(j) \\
= & \sum_{c=0}^{2 m} \eta^{c}\left(P_{2,2 m+1-c} \zeta^{j-m-1}\right) B_{m}(j) \\
= & \eta^{2 m}\left(P_{2,1} \zeta^{j-m-1} B_{m}(j)\right)+\ldots+\eta^{m+2}\left(P_{2, m-1} \zeta^{j-m-1} B_{m}(j)\right) \\
& +\eta^{m+1}\left(P_{2, m} \zeta^{j-m-1} B_{m}(j)\right)+\ldots+P_{2, k} \zeta^{j-m-1} B_{m}(j) .
\end{aligned}
$$

Da für $2 \leq i \leq m$ mit $c \in \mathbb{N}$ gilt

$$
\begin{aligned}
& \beta_{(m)} q_{m, i-2}(j-m+c+i-2 ; m+2-i) \\
& = \begin{cases}\eta^{m+i} \zeta^{j-m-1+c} B_{i-2}(j+i-m-2+c) & \text { falls } 0 \leq j+i-m-2+c \leq i-2 \\
0 & \text { sonst, }\end{cases}
\end{aligned}
$$

erhalten wir

Satz 4.43. $Z u \zeta^{j} \in H^{0}\left(\mathbb{P}^{1}, \mathcal{O}(m)\right)$ liegt

$$
\begin{aligned}
w_{m}(j):= & q_{m, m}(j ; 0) \\
& -\sum_{i=2}^{m}\left(\sum_{c=0}^{m-i+2} P_{2, m-i+1}(c) \frac{B_{m}(j) q_{m, i-2}(j-m+c+i-2 ; m+2-i)}{B_{i-2}(j+i-m-2+c)}\right)
\end{aligned}
$$

im Kern von $\beta_{(m)}$. 


\section{Kapitel 5}

\section{Das asymptotische Verhalten im festen Punkt $\mathcal{O}(m)$}

Sei erneut $(A, B) \in\left(\mathrm{gl}_{k} \times \mathrm{gl}_{k}\right) / G L_{k}$ mit nichtsingulärer Spektralkurve $S$. Wir untersuchen im Folgenden das asymptotische Verhalten der Äquivalenzklassen von Paaren von Matrizen $(A(z), B(z))$ zum Fluss $L^{z}(m)$ in $J^{k+g-1}(S)$ definiert durch die tangentiale Richtung $\left.\beta=\frac{\eta^{2}}{\zeta} \in H^{1}(S, \mathcal{O})\right)$. Allgemein erhält man für ein Geradenbündel $L \in J^{k+g-1}(S) \backslash \Theta$ und $L(-1):=L \otimes \pi_{S}^{*} \mathcal{O}_{\mathbb{P}^{1}}(-1) \subset$ $J^{g-1}(S) \backslash \Theta$ die Endomorphismen $\widetilde{A}$ und $\widetilde{B}$ von $H^{0}(S, L)$ durch Satz 1.14:

Satz. Sei mult : $H^{0}\left(S, \pi_{S}^{*} \mathcal{O}_{\mathbb{P}^{1}}(1)\right) \otimes H^{0}(S, L) \rightarrow H^{0}(S, L(1))$ die Multiplikationsabbildung. Dann ist der Homomorphismus $h: \operatorname{Ker}($ mult $) \rightarrow H^{0}(S, L)$, der $\eta \otimes s_{0}+1 \otimes s_{1}+\zeta \otimes s_{2}$ mit $s_{0}, s_{1}$ und $s_{2}$ aus $H^{0}(S, L)$ auf $s_{0}$ abbildet, ein Isomorphismus.

$\widetilde{A}$ und $\widetilde{B}$ sind folglich Endomorphismen von $H^{0}(S, L)$ mit $\eta \sigma+\widetilde{A} \sigma+\zeta \widetilde{B} \sigma=0$ für $\sigma \in H^{0}(S, L)$.

In Kapitel 4 haben wir ein Vektorbündel $V$ über $\mathbb{C}$ definiert, für das die Faser $V_{z}$ gleich $H^{0}\left(S, L^{z}(m)\right)$ mit $m=\frac{1}{2}(k-1)$ für allgemeines $z \neq 0$ ist. In diesem Kapitel untersuchen wir die Familie von Multiplikationsabbildungen

$$
H^{0}(S, \mathcal{O}(1)) \otimes V_{z} \stackrel{\text { mult }_{z}}{\longrightarrow} H^{0}\left(S, L^{z}(m+1)\right)
$$

und das asymptotische Verhalten der dadurch definierten Endomorphismen $\widetilde{A(z)}, \widetilde{B(z)}$ von $V_{z}$ für $z \rightarrow 0$. Hierzu definieren wir in Abschnitt 5.1 analog zum Bündel $V$ ein „Kernbündel“ $K$ über $\mathbb{C}$, für dessen Faser $K_{z}$ für allgemeines $z \neq 0$ gilt $K_{z}=\operatorname{Ker}\left(\right.$ mult $\left._{z}\right)$. In Abschnitt 5.2 beschreiben wir die Faser $K_{0} \subset H^{0}(S, \mathcal{O}(1)) \otimes H^{0}(S, \mathcal{O}(m))$ genauer. Dies sind genau die Elemente, für die Fortsetzungen zu Elementen aus $K_{z}$ für $z \neq 0$ und $|z|$ hinreichend klein 


\section{Kapitel 5. Das asymptotische Verhalten im festen Punkt $\mathcal{O}(\mathrm{m})$}

existieren. Elemente aus $K_{0}$ sind von der Form

$$
s_{w, v}=\eta \otimes s_{\eta}^{(w, v)}+1 \otimes s_{e}^{(w, v)}+\zeta \otimes s_{\zeta}^{(w, v)}
$$

mit $w \in H^{0}\left(\mathbb{P}^{1}, \mathcal{O}(m)\right)$ und $v \in H^{0}\left(\mathbb{P}^{1}, \mathcal{O}(m-1)\right)$. Zu einem Element $s_{w, v} \in K_{0}$ betrachten wir in Abschnitt 5.4 eine Fortsetzung zu $s_{w, v}(z) \in K_{z}$. Für $z \neq 0$ und $|z|$ hinreichend klein können wir Satz 1.14 anwenden. Die Endomorphismen $\widetilde{A(z)}$ und $\widetilde{B(z)}$ erfüllen

$$
\eta s_{\eta}^{(w, v)}(z)+\widetilde{A(z)} s_{\eta}^{(w, v)}(z)+\widetilde{B(z)} s_{\eta}^{(w, v)}(z)=0 .
$$

Es ist $\widetilde{A(z)} s_{\eta}^{(w, v)}(z)=s_{e}^{(w, v)}(z)$ und $\widetilde{B(z)} s_{\eta}^{(w, v)}(z)=s_{\zeta}^{(w, v)}(z)$, und es gilt jeweils $\lim _{z \rightarrow 0} s_{i}^{(w, v)}(z)=s_{i}^{(w, v)}(0)$ für $i=\eta, e, \zeta$. Dadurch können wir das asymptotische Verhalten von $\widetilde{A(z)}$ und $\widetilde{B(z)}$ auf $V_{z}$ bestimmen.

\subsection{Definition des Kernbündels}

Wir definieren das Kernbündel ähnlich wie Hitchin in [Hi83] für Nahmgleichungen. Nach Satz 2.4 ist $H^{0}(S, \mathcal{O}(1)) \cong H^{0}(R, \mathcal{O}(1))$ dreidimensional, und es gilt $H^{0}(S, \mathcal{O}(1))=\eta H^{0}\left(\mathbb{P}^{1}, \mathcal{O}\right) \oplus H^{0}\left(\mathbb{P}^{1}, \mathcal{O}(1)\right)$. Wir betrachten die kanonische Einbettung

$$
\begin{aligned}
\iota: R & \rightarrow \mathbb{P}^{2} \\
p & \mapsto(\eta(p): 1(p): \zeta(p))
\end{aligned}
$$

definiert durch die Basis $\eta \in \eta H^{0}\left(\mathbb{P}^{1}, \mathcal{O}\right), 1, \zeta \in H^{0}\left(\mathbb{P}^{1}, \mathcal{O}(1)\right)$ der holomorphen Schnitte des Geradenbündels $\pi_{R}^{*} \mathcal{O}_{\mathbb{P}^{1}}(1)$ über $R$. Hierbei bezeichnen wir wie in Kapitel 4 einen Schnitt aus $H^{0}(R, \mathcal{O}(1))$ durch seinen Repräsentanten über der offenen Menge $\widetilde{U_{0}}$. Das zugehörige Linearsystem hat keine Basispunkte. Für das Hyperebenenbündel $\mathcal{O}_{\mathbb{P}^{2}}(1)$ über $\mathbb{P}^{2}$ folgt $\iota^{*}\left(\mathcal{O}_{\mathbb{P}^{2}}(1)\right)=\pi_{R}^{*} \mathcal{O}_{\mathbb{P}^{1}}(1)$. Die Eulersequenz

$$
0 \rightarrow \Omega_{\mathbb{P}^{2}}^{1}(1) \rightarrow\left(\mathbb{C}^{3}\right)^{*} \rightarrow \mathcal{O}_{\mathbb{P}^{2}}(1) \rightarrow 0
$$

liefert wegen der kanonischen Isomorphie

$$
\left(\mathbb{C}^{3}\right)^{*} \cong H^{0}\left(\mathbb{P}^{2}, \mathcal{O}_{\mathbb{P}^{2}}(1)\right) \cong H^{0}\left(R, \pi_{R}^{*} \mathcal{O}_{\mathbb{P}^{1}}(1)\right) \cong H^{0}\left(\hat{R}, E_{0}\right) \stackrel{2.4}{\cong} H^{0}\left(S, \mathcal{O}_{\mathbb{P}^{1}}(1)\right)
$$

via tensorieren mit $L^{z}(m)$ die kurze exakte Sequenz über $S$

$$
\left.0 \rightarrow \Omega_{\mathbb{P}^{2}}^{1}\right|_{S} L^{z}(m+1) \rightarrow H^{0}\left(S, \mathcal{O}_{\mathbb{P}^{1}}(1)\right) \otimes L^{z}(m) \rightarrow L^{z}(m+1) \rightarrow 0 .
$$


Hierbei identifizieren wir $S \subset R$ mit $\iota(S) \subset \mathbb{P}^{2} . \iota(S)$ ist gleich der in [E03] definierten Spektralkurve $\Sigma=\left\{\left(\nu_{1}: \nu_{2}: \nu_{3}\right) \in \mathbb{P}^{2} \mid \operatorname{det}\left(\nu_{1} \mathbb{1}_{k}+\nu_{2} A+\nu_{3} B\right)=0\right\}$ in $\mathbb{P}^{2}$. Wir erhalten die lange exakte Sequenz

$$
\begin{aligned}
& 0 \rightarrow H^{0}\left(S,\left.\Omega_{\mathbb{P}^{2}}^{1}\right|_{S} L^{z}(m+1)\right) \rightarrow H^{0}\left(S, \mathcal{O}_{\mathbb{P}^{1}}(1)\right) \otimes H^{0}\left(S, L^{z}(m)\right) \stackrel{\text { mult }_{z}}{\longrightarrow} \\
& H^{0}\left(S, L^{z}(m+1)\right) \quad \rightarrow H^{1}\left(S,\left.\Omega_{\mathbb{P}^{2}}^{1}\right|_{S} L^{z}(m+1)\right) \rightarrow \quad \ldots
\end{aligned}
$$

Für $z \neq 0,|z|$ hinreichend klein, ist der $\operatorname{Kern} H^{0}\left(S, \Omega^{1} L^{z}(m+1)\right)$ der Multiplikationsabbildung

$$
\operatorname{mult}_{z}: H^{0}(S, \mathcal{O}(1)) \otimes H^{0}\left(S, L^{z}(m)\right) \rightarrow H^{0}\left(S, L^{z}(m+1)\right)
$$

isomorph zur Faser $V_{z}$ (siehe Satz 1.14). Allgemein betrachten wir das Geradenbündel $W$,

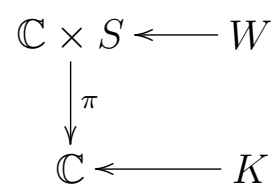

mit Faser $W_{(z, p)}=\left.\left.\Omega_{\mathbb{P}^{2}}^{1}\right|_{S} L^{z}(m+1)\right|_{p}$ und definieren das Vektorbündel $K \rightarrow \mathbb{C}$ als Bündel definiert durch die direkte Bildgarbe $\pi_{*} W$. Dann ist das Bündel $K$ vom Rang $k$ mit Faser $K_{z}=H^{0}\left(S, \Omega^{1} L^{z}(m+1)\right)$ für allgemeines $z \neq 0$.

\subsection{Berechnung von $K_{0}$}

Wir beschreiben nun $K_{0} \subset H^{0}\left(S, \Omega^{1}(m+1)\right) \subset H^{0}(S, \mathcal{O}(1)) \otimes H^{0}(S, \mathcal{O}(m))$ genauer.

Nach Definition von $K$ besteht $K_{0}$ aus den Schnitten $s \in H^{0}(S, \mathcal{O}(1)) \otimes V_{0}$, die für $z=0$ im Kern der Multiplikationsabbildung mult ${ }_{z}$ liegen und sich lokal zu Elementen $s(z)$ aus $K_{z}$ fortsetzen lassen, d.h.

$$
\begin{array}{r}
K_{0}=\left\{s \in H^{0}(S, \mathcal{O}(1)) \otimes V_{0} \mid \exists \varepsilon>0: \exists s(z) \in H^{0}(S, \mathcal{O}(1)) \otimes V_{z}\right. \text { mit } \\
s(z) \text { holom. in } z, s(0)=s \text { und } \operatorname{mult}(s(z))=0 \forall|z|<\epsilon\} .
\end{array}
$$

Diese Fortsetzung ist $\operatorname{sl}(2, \mathbb{C})$-äquivariant. Bezeichne $P_{i}$ den Raum der homogenen Polynome in zwei Variablen vom Grad $i$. Für die abstrakten Darstellungsräume von $\operatorname{sl}(2, \mathbb{C})$ gilt mit Satz $2.4, H^{0}(S, \mathcal{O}(1))=\eta H^{0}\left(\mathbb{P}^{1}, \mathcal{O}\right) \oplus$ $H^{0}\left(\mathbb{P}^{1}, \mathcal{O}(1)\right)=\eta P_{0} \oplus P_{1}$, und Satz 4.35, $V_{0}=H^{0}\left(\mathbb{P}^{1}, \mathcal{O}(m)\right) \oplus \eta H^{0}\left(\mathbb{P}^{1}, \mathcal{O}(m-\right.$ 


\section{Kapitel 5. Das asymptotische Verhalten im festen Punkt $\mathcal{O}(m)$}

$$
\begin{aligned}
1)) & =P_{m} \oplus \eta P_{m-1}: \\
K_{0} & \subset H^{0}(S, \mathcal{O}(1)) \otimes V_{0} \\
& \simeq\left(\eta H^{0}\left(\mathbb{P}^{1}, \mathcal{O}\right) \oplus H^{0}\left(\mathbb{P}^{1}, \mathcal{O}_{\mathbb{P}^{1}}(1)\right)\right) \otimes\left(H^{0}\left(\mathbb{P}^{1}, \mathcal{O}_{\mathbb{P}^{1}}(m)\right) \oplus \eta H^{0}\left(\mathbb{P}^{1}, \mathcal{O}_{\mathbb{P}^{1}}(m-1)\right)\right) \\
& =\left(\eta P_{0} \oplus P_{1}\right) \otimes\left(P_{m} \oplus \eta P_{m-1}\right) \\
& =\eta P_{m} \oplus \eta^{2} P_{m-1} \oplus\left(P_{1} \otimes P_{m}\right) \oplus\left(\eta P_{1} \otimes P_{m-1}\right) \\
& \simeq \eta P_{m} \oplus \eta^{2} P_{m-1} \oplus P_{m-1} \oplus P_{m+1} \oplus \eta P_{m-2} \oplus \eta P_{m} \\
& \simeq P_{m+1} \oplus \eta P_{m} \oplus \eta^{2} \mathbb{P}_{m-1} \oplus \eta^{3} P_{m-2} \oplus\left(P_{m} \oplus \eta P_{m-1}\right) .
\end{aligned}
$$

Für das Bild Im(mult $)_{0}$ folgt

$$
\begin{aligned}
\operatorname{Im}\left(\text { mult }_{0}\right) & \subset H^{0}(S, \mathcal{O}(m+1)) \\
& \stackrel{2.4}{=} \bigoplus_{i=0}^{m+1} \eta^{i} H^{0}\left(\mathbb{P}^{1}, \mathcal{O}_{\mathbb{P}^{1}}(m+1-i)\right) \\
& =P_{m+1} \oplus \eta P_{m} \oplus \eta^{2} P_{m-1} \oplus \eta^{3} P_{m-2} \oplus \ldots \oplus \eta^{m+1} P_{0} .
\end{aligned}
$$

Damit ist $\left.\operatorname{Ker}\left(\right.$ mult $\left._{0}\right)\right|_{H^{0}(S, \mathcal{O}(1)) \otimes V_{0}}$ isomorph zu $P_{m} \oplus \eta P_{m-1}$. Konkret ist $P_{m} \oplus$ $\eta P_{m-1}$ in $H^{0}(S, \mathcal{O}(1)) \otimes V_{0}$ durch eine $s l(2, \mathbb{C})$-äquivariante nichttriviale Abbildung gegeben. Wir definieren die folgenden Abbildungen, wobei wir mit $\zeta^{i}$ mit $0 \leq i \leq m-1$ bzw. $\zeta^{j}$ mit $0 \leq j \leq m$ jeweils die Standardbasen von $P_{m-1}$ bzw. $P_{m}$ bezeichnen: Sei

$$
\mathfrak{k}_{m}: P_{m} \rightarrow P_{1} \otimes \eta P_{m-1}
$$

definiert durch

$$
\zeta^{j} \mapsto-1 \otimes(m-j) \eta \zeta^{j}-\zeta \otimes j \eta \zeta^{j-1}
$$

und

$$
\mathfrak{k}_{m-1}: \eta P_{m-1} \rightarrow P_{1} \otimes P_{m}
$$

definiert durch

$$
\eta \zeta^{i} \mapsto 1 \otimes \zeta^{i+1}-\zeta \otimes \zeta^{i}
$$

Diese Abbildungen sind nichttrivial, $s l_{2}(\mathbb{C})$-äquivariant und nach dem Lemma von Schur eindeutig bis auf skalare Vielfache. Sie definieren eine $s l_{2}(\mathbb{C})$-äquivariante Abbildung

$$
\mathfrak{k}: P_{m} \oplus \eta P_{m-1} \rightarrow\left(\eta P_{0} \oplus P_{1}\right) \otimes\left(P_{m} \oplus \eta P_{m-1}\right)
$$

durch

$$
\begin{gathered}
\left(\zeta^{j}, \eta \zeta^{i}\right) \mapsto \eta \otimes m \zeta^{j}+1 \otimes\left(\zeta^{i+1}-(m-j) \eta \zeta^{j}\right)+\zeta \otimes\left(-\zeta^{i}-j \eta \zeta^{j-1}\right) \\
=\eta \otimes m \zeta^{j}+\mathfrak{k}_{m}\left(\zeta^{j}\right)+\mathfrak{k}_{m-1}\left(\eta \zeta^{i}\right) .
\end{gathered}
$$


$\mathfrak{k}$ ist $s l_{2}(\mathbb{C})$-äquivariant, und es gilt $\operatorname{mult}_{0}\left(\mathfrak{k}\left(P_{m} \oplus \eta P_{m-1}\right)\right)=0$.

Wir definieren Abbildungen $X_{e}, X_{\zeta}, Y_{e}$ und $Y_{\zeta}$ wie folgt:

$$
\begin{aligned}
X_{e}: P_{m-1} & \rightarrow P_{m} \backslash<1>, \\
\zeta^{j} & \mapsto \zeta^{j+1} ; \\
X_{\zeta}: P_{m-1} & \rightarrow P_{m} \backslash<\zeta^{m}>, \\
\zeta^{j} & \mapsto-\zeta^{j} ; \\
Y_{e}: P_{m} & \rightarrow P_{m-1}, \\
\zeta^{j} & \mapsto-m \zeta^{j}+j \zeta^{j}=(j-m) \zeta^{j} \\
\text { und damit } \operatorname{ker}\left(Y_{e}\right) & =<\zeta^{m}>; \\
Y_{\zeta}: P_{m} & \rightarrow P_{m-1}, \\
\zeta^{j} & \mapsto-j \zeta^{j-1}
\end{aligned}
$$

und damit $\operatorname{ker}\left(Y_{\zeta}\right)=<1>$.

Bezüglich der Standardbasen von $P_{m}$ und $P_{m-1}$ haben die zugehörigen Matrizen die Form

$$
\begin{aligned}
& X_{e}=\left(\begin{array}{cccc}
0 & 0 & \cdots & 0 \\
1 & 0 & & \vdots \\
0 & 1 & & \\
\vdots & \vdots & & \\
0 & 0 & \cdots & 1
\end{array}\right) \in \mathrm{M}((m+1) \times m, \mathbb{C}) \\
& X_{\zeta}=\left(\begin{array}{cccc}
-1 & 0 & \cdots & 0 \\
0 & -1 & & \vdots \\
0 & 0 & & \\
\vdots & \vdots & & -1 \\
0 & 0 & \cdots & 0
\end{array}\right) \in \mathrm{M}((m+1) \times m, \mathbb{C}), \\
& Y_{e}=\left(\begin{array}{ccccc}
-m & 0 & \cdots & 0 & 0 \\
0 & 1-m & & \vdots & \vdots \\
\vdots & \vdots & & 0 & \vdots \\
0 & 0 & \cdots & -1 & 0
\end{array}\right) \in \mathrm{M}(m \times(m+1), \mathbb{C})
\end{aligned}
$$

und 


$$
\begin{gathered}
Y_{\zeta}=\left(\begin{array}{ccccc}
0 & -1 & \cdots & 0 & 0 \\
0 & 0 & & \vdots & \vdots \\
\vdots & \vdots & & -m+1 & \vdots \\
0 & 0 & \cdots & 0 & -m
\end{array}\right) \in \mathrm{M}(m \times(m+1), \mathbb{C}) . \\
\mathfrak{k}: H^{0}\left(\mathbb{P}^{1}, \mathcal{O}(m)\right) \oplus \eta H^{0}\left(\mathbb{P}^{1}, \mathcal{O}(m)\right) \rightarrow K_{0} \subset H^{0}(S, \mathcal{O}(1)) \otimes V_{0}
\end{gathered}
$$

hat dann die Gestalt

$$
\mathfrak{k}(w, \eta v)=\eta \otimes m w+1 \otimes\left(X_{e}(v)+\eta Y_{e}(w)\right)+\zeta \otimes\left(X_{\zeta}(v)+\eta Y_{\zeta}(w)\right) .
$$

Da die Abbildung $\mathfrak{k}$ injektiv ist und der Rang des Vektorbündels $K$ gleich $k$ ist, folgt

Satz 5.1. Jedes Element aus $K_{0}$ kann eindeutig in der Form

$$
\mathfrak{k}(w, \eta v)=\eta \otimes m w+1 \otimes\left(X_{e}(v)+\eta Y_{e}(w)\right)+\zeta \otimes\left(X_{\zeta}(v)+\eta Y_{\zeta}(w)\right)
$$

mit Elementen $w \in H^{0}\left(\mathbb{P}^{1}, \mathcal{O}(m)\right)$ und $v \in H^{0}\left(\mathbb{P}^{1}, \mathcal{O}(m-1)\right)$ geschrieben werden.

\subsection{Erweiterungen von Elementen aus $K_{0}$}

Wir haben im vorigen Abschnitt $K_{0} \subset H^{0}(S, \mathcal{O}(1)) \otimes V_{0}$ bestimmt. Zu Elementen $s^{(w, v)}=\mathfrak{k}(w, v) \in K_{0}$ gibt es folglich holomorph von $z$ abhängige Schnitte $s^{(w, v)}(z)$ in $K_{z}=\operatorname{ker}\left(\right.$ mult $\left._{z}\right) \subset H^{0}(S, \mathcal{O}(1)) \otimes H^{0}\left(S, L^{z}(m)\right)$ für $|z|$ hinreichend klein. Wie für das Vektorbündel $V$ definieren wir eine Fortsetzung von $s^{(w, v)}$ zur Ordnung $l$ als ein Element der Form

$$
\begin{aligned}
\sigma^{l}:=\eta & \otimes\left(s_{\eta, 0}^{(w, v)}+z s_{\eta, 1}^{(w, v)}+\cdots+z^{l} s_{\eta, l}^{(w, v)}\right) \\
& +1 \otimes\left(s_{e, 0}^{(w, v)}+\cdots+z^{l} s_{e, 0}^{(w, v)}\right) \\
& +\zeta \otimes\left(s_{\zeta, 0}^{(w, v)}+\cdots+z^{l} s_{\zeta, 0}^{(w, v)}\right)
\end{aligned}
$$

mit

$$
\begin{aligned}
\operatorname{mult}_{z}\left(\sigma^{l}\right)= & \left(\eta s_{\eta, 0}^{(w, v)}+s_{e, 0}^{(w, v)}+\zeta s_{\zeta, 0}^{(w, v)}\right)+z\left(\eta s_{\eta, 1}^{(w, v)}+s_{e, 1}^{(w, v)}+\zeta s_{\zeta, 1}^{(w, v)}\right) \\
& +\cdots+z^{l}\left(\eta s_{\eta, l}^{(w, v)}+s_{e, l}^{(w, v)}+\zeta s_{\zeta, l}^{(w, v)}\right) \\
= & 0 .
\end{aligned}
$$


Dies entspricht einem Element $\sigma^{l}$ aus $H^{0}(S, \mathcal{O}(1)) \otimes H^{0}\left(S, F^{l}(m)\right)$, für das gilt $\operatorname{mult}_{z}\left(\sigma^{l}\right)=0 \in H^{0}\left(S, F^{l+1}(m)\right)$ und $\operatorname{pr}_{0}\left(\sigma^{l}\right)=s^{(w, v)}$, wobei die Projektionsabbildung $\mathrm{pr}_{0}: H^{0}(S, \mathcal{O}(1)) \otimes H^{0}\left(S, F^{l}(m)\right) \rightarrow H^{0}(S, \mathcal{O}(1)) \otimes H^{0}(S, \mathcal{O}(m))$ durch $\operatorname{pr}_{0}: H^{0}\left(S, F^{l}(m)\right) \rightarrow H^{0}(S, \mathcal{O}(m))$ induziert sei.

Eine Basis von $K_{0}$ ist gegeben durch

$$
\begin{gathered}
\left\{1 \otimes \zeta^{i+1}-\zeta \otimes \zeta^{i} \mid 0 \leq i \leq m-1\right\} \\
\bigcup\left\{\eta \otimes m \zeta^{j}-1 \otimes(m-j) \eta \zeta^{j}-\zeta \otimes\left(j \eta \zeta^{j-1}\right) \mid 0 \leq j \leq m\right\} .
\end{gathered}
$$

Hierbei sind $\zeta^{i+1}=X_{e}\left(\zeta^{i}\right)$ und $\zeta^{i}=-X_{\zeta}\left(\zeta^{i}\right)$ Elemente aus $H^{0}\left(\mathbb{P}^{1}, \mathcal{O}(m)\right)$ und entsprechend $(m-j) \eta \zeta^{j}=-\eta Y_{e}\left(\zeta^{j}\right)$ und $j \eta \zeta^{j-1}=-\eta Y_{\zeta}\left(\zeta^{j}\right)$ Elemente aus $\eta H^{0}\left(\mathbb{P}^{1}, \mathcal{O}(m-1)\right)$. Fortsetzungen der Basiselemente erhält man nun, indem man die zugehörigen Elemente aus $H^{0}\left(\mathbb{P}^{1}, \mathcal{O}(m)\right)$ bzw. $\zeta H^{0}\left(\mathbb{P}^{1}, \mathcal{O}(m-1)\right)$ wie in Kapitel 4 fortsetzt und den Term bei $\eta$ so wählt, dass die Summe im Kern der Multiplikationsabbildung liegt. Z.B. gilt mit den Bezeichnungen aus Kapitel 4:

Satz 5.2. Seien $l<m$ und $0 \leq i \leq m-1$ natürliche Zahlen. Dann sind

$$
\eta \otimes\left(-\frac{1}{m} q_{l, m-1}(i ; 1)\right)+1 \otimes q_{l, m}(i+1 ; 0)-\zeta \otimes q_{l, m}(i ; 0)
$$

Elemente in $H^{0}(S, \mathcal{O}(1)) \otimes H^{0}\left(S, F^{l}(m)\right)$, deren Bilder unter der Projektionsabbildung $\mathrm{pr}_{0}$ gleich $1 \otimes \zeta^{i+1}-\zeta \otimes \zeta^{i}$ sind und die im Kern der Abbildung mult $_{z}$ liegen.

Beweis: Es gilt $q_{l, m-1}(i ; 1) \in H^{0}\left(S, F^{l}(m)\right)$. Außerdem sind $q_{l, m}(i+1 ; 0)$ und $q_{l, m}(i ; 0)$ Elemente in $H^{0}\left(S, F^{l}(m)\right)$ mit $\operatorname{pr}_{0}\left(q_{l, m}(i+1 ; 0)\right)=\zeta^{i+1}$ und $\operatorname{pr}_{0}\left(q_{l, m}(i ; 0)\right)=\zeta^{i}$. Mit

$$
\begin{aligned}
\eta^{-1} & \left(q_{l, m}(i+1 ; 0)-\zeta q_{l, m}(i ; 0) \text { an der Stelle } 0 \leq c \leq l\right) \\
& =\eta^{-1}\left(\eta^{2 c}\left(f_{c, m}(i+1)-f_{c, m}(i)\right) \zeta^{i+1-c}\right) \\
& =\eta^{1+2(c-1)} \frac{i !(m-c) !}{(c-1) !(i+1-c) ! m !} \zeta^{i+1-c} \\
& =\frac{1}{m} \eta^{1+2(c-1)} f_{c-1, m-1}(i) \zeta^{i+1-c} \\
& =\frac{1}{m}\left(q_{l, m-1}(i ; 1) \text { an der Stelle } 0 \leq c \leq l\right)
\end{aligned}
$$

folgt die Behauptung. 


\section{Kapitel 5. Das asymptotische Verhalten im festen Punkt $\mathcal{O}(\mathrm{m})$}

\subsection{Berechnung der Polordnungen und Resi- duen}

Die Wahl von Basen $w_{j}=\zeta^{j}$ von $H^{0}\left(\mathbb{P}^{1}, \mathcal{O}(m)\right)$ mit $0 \leq j \leq m$ und $v_{i}=\eta \zeta^{i}$ von $\eta H^{0}\left(\mathbb{P}^{1}, \mathcal{O}(m-1)\right)$ mit $0 \leq i \leq m-1$ definiert eine Basis von $V_{0}$. Diese Basiselemente können wir zu Schnitten $w_{j}(z), v_{i}(z) \in H^{0}\left(S, L^{z}(m)\right)$ fortsetzen. Für hinreichend kleines $|z|$ sind $w_{j}(z)$ und $v_{i}(z)$ ebenfalls eine Basis in $H^{0}\left(S, L^{z}(m)\right)$. Wir erhalten durch $w_{j}, v_{i}$ somit eine Rahmung des Vektorbündels $V$ über einer kleinen Umgebung von $0 \in \mathbb{C}$. Verschiedene Fortsetzungen von $w_{j}$ und $v_{i}$ definieren lediglich verschiedene Trivialisierungen von $V$ über einer Umgebung von $0 \in \mathbb{C}$. Da wir nur Funktionen auf Äquivalenzklassen von Paaren von Matrizen, $\left(\mathrm{gl}_{k} \times \mathrm{gl}_{k}\right) / \mathrm{GL}_{k}$, betrachten, können wir ohne Einschränkung zu jedem Basisvektor aus $V_{0}$ eine Fortsetzung und damit eine Rahmung fixieren. Wir wählen hier Fortsetzungen zu $w_{j}$ bzw. $v_{i}$, die wir durch induktive Korrekturen der kanonischen Fortsetzungen $q_{m, m}(j ; 0)$ von $w_{j}$ zur Ordnung $m$ bzw. $q_{m-1, m-1}(i ; 0)$ von $v_{i}$ zur Ordnung $m-1$ wie in Abschnitt 4.12 erhalten.

Wir betrachten nun das asymptotische Verhalten der durch Satz 1.14 gegebenen Endomorphismen $\widetilde{A}=\widetilde{A(z)}$ und $\widetilde{B}=\widetilde{B(z)}$ von $H^{0}\left(S, L^{z}(m)\right)$ bzw. das Verhalten der zugehörigen Matrizen bezüglich einer fest gewählten Rahmung. Seien $\eta \zeta^{i} \in H^{0}\left(\mathbb{P}^{1}, \mathcal{O}(m-1)\right)$ und $\zeta^{j} \in H^{0}\left(\mathbb{P}^{1}, \mathcal{O}(m)\right)$ Basen. Da $X_{e, \zeta}\left(\zeta^{i}\right) \in H^{0}\left(\mathbb{P}^{1}, \mathcal{O}(m)\right)$ sind, gibt es Fortsetzungen zu $X_{e, \zeta}\left(\zeta^{i}\right)$, deren ersten beiden Komponenten mit den Einträgen der kanonischen Fortsetzungen von $X_{e, \zeta}\left(\zeta^{i}\right)$ zur Ordnung $m$ übereinstimmen. Dies folgt aus Bemerkung 4.40, nach der die $q_{m, m}(j ; 0)$ mit $0 \leq j \leq m$ Elemente von $H^{0}\left(S, F^{m}(m)\right)$ sind, und Satz 4.42 , wonach für höhere Fortsetzungen nötige Korrekturterme die ersten beiden Einträge von $q_{m, m}(j ; 0)$ unverändert lassen. Mit Satz 5.2 folgt, dass jede Fortsetzung des Basiselementes $1 \otimes \zeta^{i+1}-\zeta \otimes \zeta^{i}$ mit $0 \leq i \leq m-1$ von der Form

$$
\begin{aligned}
\eta \otimes\left(-\frac{1}{m} z \eta \zeta^{i}+z^{2}(*)\right)+1 \otimes\left(\zeta^{i+1}\right. & \left.+z \eta^{2}\left(\frac{i+1}{m} \zeta^{i}\right)+z^{2}(*)\right) \\
& +\zeta \otimes\left(-\zeta^{i}-z \eta^{2}\left(\frac{i}{m} \zeta^{i-1}\right)+z^{2}(*)\right)
\end{aligned}
$$

ist. Wir erhalten:

$$
\begin{aligned}
\lim _{z \rightarrow 0} z \widetilde{A}\left(\eta \zeta^{i}\right) & =\lim _{z \rightarrow 0} \widetilde{A}\left(z \eta \zeta^{i}\right) \\
& =\lim _{z \rightarrow 0}-m \widetilde{A}\left(z \eta\left(-\frac{1}{m}\right) \zeta^{i}\right) \\
& =-m \zeta^{i+1}
\end{aligned}
$$


und analog

$$
\begin{aligned}
\lim _{z \rightarrow 0} z \widetilde{B}\left(\eta \zeta^{i}\right) & =\lim _{z \rightarrow 0}-m \widetilde{B}\left(z \eta\left(-\frac{1}{m}\right) \zeta^{i}\right) \\
& =m \zeta^{i} .
\end{aligned}
$$

Außerdem gilt

$$
\begin{aligned}
\lim _{z \rightarrow 0} \widetilde{A}\left(\zeta^{j}\right) & =\lim _{z \rightarrow 0} \frac{1}{m} \widetilde{A}\left(m \zeta^{j}\right) \\
& =-\frac{1}{m}(m-j) \eta \zeta^{j}
\end{aligned}
$$

und

$$
\lim _{z \rightarrow 0} \widetilde{B}\left(\zeta^{j}\right)=-\frac{1}{m} j \eta \zeta^{j-1}
$$

Es folgt

Satz 5.3. Die Endomorphismen $\widetilde{A}$ und $\widetilde{B}$ von $V_{z}$ haben in $z=0$ einen Pol erster Ordnung.

Bezüglich der gewählten Rahmung gilt für die Residuen der Matrizen $A, B$ zu den Endomorphismen $\widetilde{A}, \widetilde{B}$ :

$$
\operatorname{Res}(A)=\left(\begin{array}{cccc} 
& 0 & \ldots & 0 \\
0_{m+1} & & & \\
& & -m \mathbb{1}_{m} & \\
\hline 0_{m \times(m+1)} & & & \\
& & &
\end{array}\right)
$$

und

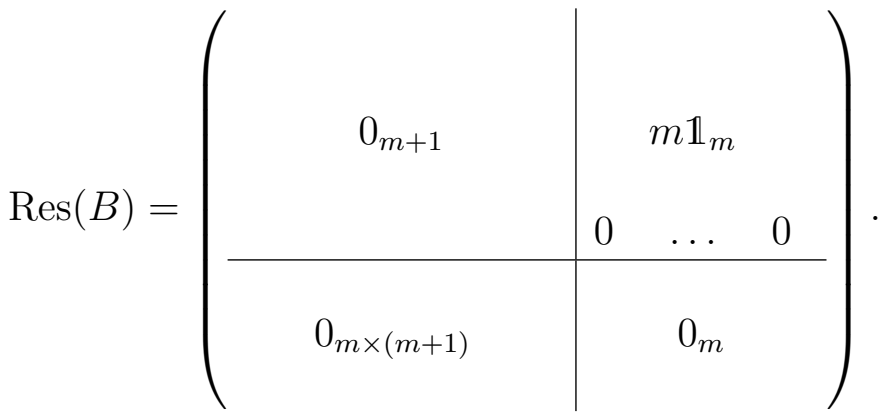


96 Kapitel 5. Das asymptotische Verhalten im festen Punkt $\mathcal{O}(m)$ 


\section{Kapitel 6}

\section{Beschreibung mittels Thetafunktionen}

Wir stellen nun einen Algorithmus zur Beschreibung von konjugationsinvarianten Funktionen auf Paaren von Matrizen mittels Thetafunktionen vor. Sei $f \in \mathbb{C}\left[\mathrm{gl}_{k} \times \mathrm{gl}_{k}\right]^{\mathrm{GL}_{k}}$. Sei $(A, B) \in\left(\mathrm{gl}_{k} \times \mathrm{gl}_{k}\right) / \mathrm{GL}_{k}$ fest gewählt mit nichtsingulärer Spektralkurve $S$. Die Einschränkung von $f$ auf die Menge $M_{S}$ aller Äquivalenzklassen von Paaren von Matrizen mit Spektralkurve $S$ drücken wir folgendermaßen durch Thetafunktionen auf der affinen Jakobischen $J(S) \backslash \Theta$ aus:

S 1 Im ersten Schritt bestimmen wir das Verhalten von $f$ in einer Umgebung eines allgemeinen Punktes des Thetadivisors $\Theta \subset J(S)$.

In Abschnitt 2.5 haben wir gesehen, dass jedes lineare Vektorfeld auf der Jakobischen definiert durch ein beliebiges $\beta \in H^{1}(S, \mathcal{O})$ den Thetadivisor in allgemeinen Punkten transversal schneidet. Wir wählen $\beta \in$ $H^{1}(S, \mathcal{O})$ repräsentiert durch $\frac{\eta^{2}}{\zeta}$.

Mit Satz 3.1 ist die Ordnung einer meromorphen Funktion $\hat{f}: J(S) \rightarrow \mathbb{C}$ in einem allgemeinen Punkt des Thetadivisors durch seine Ordnung entlang des Flusses definiert durch $\beta$ bestimmt.

Nach Satz 3.7 beschreibt jede strikte homogene Laurentlösung zur LaxPaar-Gleichung $\dot{\mathcal{C}}=\left[\mathcal{C}, \beta(\mathcal{C}, \zeta)^{+}\right]$mit $\mathcal{C}=A+\zeta B$ das asymptotische Verhalten von Äquivalenzklassen von Paaren von Matrizen, deren zugehöriger Fluß in der Jakobischen $J(\Sigma)$ einer festen Spektralkurve $\Sigma=S_{A(z), B(z)}$ gegen einen Punkt des Thetadivisors $\Theta \subset J(\Sigma)$ läuft. 
Nach Satz 3.18 ergeben die durch die indizierende Menge

$$
A^{(0)}=\left(\begin{array}{cccccc}
0 & 1 & 0 & 0 & \cdots & 0 \\
0 & 0 & 1 & 0 & \cdots & 0 \\
0 & \cdots & & & & 0 \\
\vdots & & & & & \vdots \\
\vdots & & & & & \vdots \\
0 & \cdots & & & & 0
\end{array}\right), B=\left(\begin{array}{cccccc}
b_{11} & & & & & \\
1 & b_{22} & & & * & \\
0 & -1 & b_{11} & 0 & \cdots & 0 \\
0 & & & & & \\
\vdots & & * & & & \\
0 & & & & & b_{k k}
\end{array}\right)
$$

definierten strikten homogenen formalen Laurentlösungen eine Hauptbalance von $\dot{\mathcal{C}}=\left[\mathcal{C}, \beta(\mathcal{C}, \zeta)^{+}\right]$und beschreiben das asymptotische Verhalten von Äquivalenzklassen von Paaren von Matrizen in allgemeinen Punkten des verallgemeinerten Thetadivisors. Durch Fixieren der Spektralkurve $S$ und damit Festlegung von $\frac{1}{2} k(k+3)$ Parametern der Lösungen $A(z), B$ hängt die zugehörige Familie von strikten formalen homogenen Laurentlösungen nur noch von $k^{2}-\frac{1}{2} k(k+3)=\frac{1}{2} k(k-3)=g-1$ Parametern ab. Sie beschreibt das asymptotische Verhalten von Äquivalenzklassen von Paaren von Matrizen entlang $\beta$ durch einen allgemeinen Punkt des Thetadivisors in der Jakobischen $J(S)$. Die fest gewählten Parameter sind durch die Koeffizienten des charakteristischen Polynoms fixiert. In Abschnitt 3.5 haben wir konstante Terme $A^{(1)}$ solcher möglichen Laurentlösungen bestimmt. Bei Bedarf können weitere Terme $A^{(l)}$ mittels Lösen des linearen Gleichungssystems (3.14), unter Umständen mithilfe eines Computers, berechnet werden. Die Anzahl der benötigten Koeffizienten $A^{(l)}$ hängt hierbei von der zu untersuchenden Funktion $f$ ab. Die berechneten strikten formalen Laurentlösungen werden in die Funktion $f$ eingesetzt und so ihr Verhalten, d.h. z.B. ihre Polordnungen und Residuen bzw. der gesamte Hauptteil der Laurentreihen in Richtung $\beta$, in einem allgemeinen Punkt des Thetadivisors bestimmt.

S 2 Im zweiten Schritt wird die Menge aller meromorphen Funktionen auf der Jakobischen $J(S)$ untersucht, deren Polordnungen in allgemeinen Punkten des Thetadivisors gleich der in Schritt 1 berechneten Polordnung ist. Hierfür verwendet man die in Abschnitt 1.3 angegebenen Thetafunktionen. Ist die Polordnung von $\hat{f}$ in einem allgemeinen Punkt von $\Theta$ gleich $n \in \mathbb{N}$, so sind alle gesuchten möglichen Funktionen gegeben durch

$$
H^{0}(J(S), \mathcal{O}(n \Theta)) \cong\{f: J(S) \rightarrow \mathbb{C} \mid f \text { meromorph },(f) \geq-n \Theta\} .
$$

$\hat{f}$ ist in diesem Fall gleich $\frac{g}{\vartheta^{n}}$ für ein $g \in R_{n}^{Z}\left(R_{n}^{Z}\right.$ ist der Raum der Thetafunktionen vom Gewicht $n$ zur Jakobischen $J(S)$ mit Periodenmatrix $(\mathbb{1}, Z)$, siehe Satz 1.10). 
Findet man eine solche Funktion $\tilde{f}:=\frac{g}{\vartheta^{n}}$, deren Hauptteil der Laurententwicklung in Richtung $\beta$ in einem allgemeinen Punkt gleich dem im ersten Schritt berechneten Hauptteil ist, so stimmen $\tilde{f}$ und $f$ auf $J(S)$ ohne einer Untervarietät der Kodimension größer oder gleich zwei überein. Damit ist ihre Differenz $\tilde{f}-f$ eine konstante Funktion.

S 3 Um den konstanten Term von $\tilde{f}-f$ zu bestimmen, müssen wir jeweils die konstanten Terme der Laurententwicklungen von $f(A(z), B(z))$ bzw. $\tilde{f}(z)$ in einem konkreten Punkt des Thetadivisors bestimmen. Im dritten Schritt führt man dies für $f(A(z), B(z))$ durch. Der einzige Punkt des Thetadivisors in der Jakobischen, der explizit bekannt ist, ist der Punkt $\mathcal{O}_{S}(m) \in \Theta \subset J^{k+g-1}(S)$. Laut Satz 5.3 haben die Endomorphismen $\widetilde{A(z)}, \widetilde{B(z)}$ von $H^{0}\left(S, L^{z}(m)\right)$ in $z=0$ einen Pol der Ordnung eins. Eine Laurententwicklung $A(z), B(z)$ (bzw. ihre ersten Koeffizienten) zu den Endomorphismen $\widetilde{A(z)}, \widetilde{B(z)}$ entlang $\beta$ kann mithilfe der in Kapitel 4 angegebenen Erweiterung von Schnitten von $H^{0}(S, \mathcal{O}(m))$ berechnet werden. Hierzu erweitert man die in Satz 5.1 gegebenen Elemente aus $K_{0}$ zu Elementen von $K_{z}$, indem man jeweils zu Basiselementen aus $V_{0}$ die kanonische Erweiterung $q_{m, m}(j ; 0)$ bzw. $q_{m, m-1}(i ; 0)$, siehe Satz 4.30, berechnet, und diese dann induktiv durch Korrekturterme aus Satz 4.42 und Definition 4.41 korrigiert. Zugehörige Elemente in $K_{z}$ erhält man dann durch die Bedingung, dass sie im Kern der Multiplikationsabbildung mult $t_{z}$ liegen müssen. Durch eine geeignete Wahl einer Rahmung von $V_{z}$ können die gesuchten Matrixpolynome bestimmt werden.

S $4 \mathrm{Im}$ vierten Schritt berechnen wir die Laurententwicklung der in Schritt 2 bestimmten Funktion $\tilde{f}$ in $\mathcal{O}_{S}(m)$ entlang $\beta$. Unter Umständen müssen wir hierzu zusätzliche Eigenschaften von $\mathcal{O}_{S}(m)$, z.B. dass $\mathcal{O}_{S}(m)$ eine Thetacharakteristik ist (siehe Abschnitt 1.5), benutzen.

$\mathrm{S} 5$ Die Differenz der Laurententwicklungen in $\mathcal{O}_{S}(m)$ entlang $\beta$ von $f$ aus Schritt 3 [S 3] und $\tilde{f}$ aus Schritt 4 [S 4] ergibt die gesuchte Konstante.

\section{Konkretes Beispiel tr $\left([A, B]^{2}\right)$}

Beispielhaft beschreiben wir die Funktion $f:=\operatorname{tr}\left([A, B]^{2}\right)$ bis auf einen konstanten Term mittels Thetafunktionen.

S 1 Hierzu betrachten wir eine allgemeine formale Laurententwicklung von $A$ und $B$ in Richtung $\beta=\frac{\eta^{2}}{\zeta}$, d.h. $A=\frac{1}{z} A^{(0)}+A^{(1)}+z A^{(2)}+\ldots, B$ konstant mit 


$$
A^{(0)}=\left(\begin{array}{cccccc}
0 & 1 & 0 & 0 & \cdots & 0 \\
0 & 0 & 1 & 0 & \cdots & 0 \\
0 & \cdots & & & & 0 \\
\vdots & & & & & \vdots \\
\vdots & & & & & \vdots \\
0 & \cdots & & & & 0
\end{array}\right), B=\left(\begin{array}{ccccccc}
b_{11} & & & & & \\
1 & b_{22} & & & * & \\
0 & -1 & b_{11} & 0 & \cdots & 0 \\
0 & & & & & \\
\vdots & & * & & & \\
0 & & & & & b_{k k}
\end{array}\right)
$$

und

$$
A^{(1)}=\left(\begin{array}{cccccc}
a_{11}^{(1)} & a_{12}^{(1)} & a_{13}^{(1)} & \ldots & & a_{1 k}^{(1)} \\
0 & -a_{11}^{(1)} & -a_{12}^{(1)} & 0 & \cdots & 0 \\
0 & 0 & a_{11}^{(1)} & 0 & \cdots & 0 \\
0 & 0 & a_{43}^{(1)} & a_{44}^{(1)} & \cdots & a_{4 k}^{(1)} \\
\vdots & \vdots & \vdots & & & \vdots \\
0 & 0 & a_{k 3}^{(1)} & \cdots & & a_{k k}^{(1)}
\end{array}\right) .
$$

Dies entspricht einer Hauptbalance, d.h. der Entwicklung von $A, B$ in einem allgemeinen Punkt des Thetadivisors, wobei durch die Koeffizienten des charakteristischen Polynoms einige der Koeffizienten $a_{i j}^{(1)}$ bzw. $b_{i j}$ fixiert sind.

Es gilt

$$
\begin{aligned}
\operatorname{tr}\left([A, B]^{2}\right)=\frac{1}{z^{2}} & \operatorname{tr}\left(\left[A^{(0)}, B\right]^{2}\right)+\frac{1}{z}\left(\operatorname{tr}\left(\left[A^{(0)}, B\right]\left[A^{(1)}, B\right]+\left[A^{(1)}, B\right]\left[A^{(0)}, B\right]\right)\right) \\
& +\left(\operatorname{tr}\left(\left[A^{(1)}, B\right]^{2}+\left[A^{(0)}, B\right]\left[A^{(2)}, B\right]+\left[A^{(2)}, B\right]\left[A^{(0)}, B\right]\right)\right) \\
& +\sum_{l=1}^{\infty} z^{l}\left(\sum_{n=0}^{l+2}\left[A^{(n)}, B\right]\left[A^{(l+2-n)}, B\right]\right) .
\end{aligned}
$$

Mit

$$
\begin{aligned}
&\left(\left[A^{(0)}, B\right]^{2}\right)_{i j}=\sum_{l=1}^{k}(\left(A^{(0)} B\right)_{i l}\left(A^{(0)} B\right)_{l j}-\left(A^{(0)} B\right)_{i l}\left(B A^{(0)}\right)_{l j} \\
&\left.-\left(B A^{(0)}\right)_{i l}\left(A^{(0)} B\right)_{l j}+\left(B A^{(0)}\right)_{i l}\left(B A^{(0)}\right)_{l j}\right)
\end{aligned}
$$

und

$$
\left(A^{(0)} B\right)_{i j}=\sum_{l=1}^{k} a_{i l} b_{l j}= \begin{cases}0 & \text { falls } i \neq 1,2 \\ b_{2 j} & \text { falls } i=1 \\ b_{3 j} & \text { falls } i=2\end{cases}
$$




$$
\left(B A^{(0)}\right)_{i j}=\sum_{l=1}^{k} b_{i l} a_{l j}= \begin{cases}0 & \text { falls } j \neq 2,3 \\ b_{i 1} & \text { falls } j=2 \\ b_{i 2} & \text { falls } j=3\end{cases}
$$

folgt

$$
\begin{aligned}
& \left(\left[A^{(0)}, B\right]^{2}\right)_{i i}=0 \text { für } i \geq 4 \\
& \left(\left[A^{(0)}, B\right]^{2}\right)_{11}=b_{21}^{2}+b_{22} b_{31}-b_{11} b_{31}=1 \\
& \left(\left[A^{(0)}, B\right]^{2}\right)_{22}=4 \\
& \left(\left[A^{(0)}, B\right]^{2}\right)_{33}=1
\end{aligned}
$$

und damit

$$
\operatorname{tr}\left([A, B]^{2}\right)=\frac{6}{z^{2}}+\frac{c_{-1}}{z}+c_{0}+z c_{1}+\ldots
$$

Das Residuum $c_{-1}$ erhalten wir durch

$$
\begin{aligned}
c_{-1}= & \operatorname{tr}\left(\left[A^{(0)}, B\right]\left[A^{(1)}, B\right]+\left[A^{(1)}, B\right]\left[A^{(0)}, B\right]\right) \\
= & 2 \operatorname{tr}\left(\left[A^{(0)}, B\right]\left[A^{(1)}, B\right]\right) \\
= & 2 \operatorname{tr}\left(A^{(0)}\left(2 B A^{(1)} B-A^{(1)} B^{2}-B^{2} A^{(1)}\right)\right) \\
= & 2\left(2\left(B A^{(1)} B\right)_{21}+2\left(B A^{(1)} B\right)_{32}-\left(A^{(1)} B^{2}\right)_{21}\right. \\
& \left.\quad-\left(A^{(1)} B^{2}\right)_{32}-\left(B^{2} A^{(1)}\right)_{21}-\left(B^{2} A^{(1)}\right)_{32}\right) \\
= & 2\left(2\left(a_{11}^{(1)}\left(b_{11}-b_{22}\right)+a_{12}^{(1)}\right)+2\left(a_{11}^{(1)}\left(b_{22}-b_{11}\right)-a_{12}^{(1)}\right)+0\right) \\
= & 0 .
\end{aligned}
$$

S 2 Im Schritt [S 1] haben wir das asymptotische Verhalten von $f$ entlang der Richtung $\beta=\frac{\eta^{2}}{\zeta}$ bestimmt. Insbesondere kennen wir nun die Polordnung von $f$ entlang $\Theta$, sie ist gleich 2. Entwickeln wir die Thetafunktion $\vartheta$ in einem allgemeinen Punkt $L$ von $\Theta$ in Richtung $\beta$, so hat $\vartheta$ die Form $\vartheta=z\left(a_{0}+z a_{1}+z^{2} a_{2}+\ldots\right)$. Ein möglicher Kandidat für die gesuchte Funktion $\hat{f}: J(S) \rightarrow \mathbb{C}$ ist dann die Funktion

$$
\begin{aligned}
\frac{d^{2}}{d z^{2}} \log \vartheta & =\frac{\vartheta \frac{d^{2}}{d z^{2}}(\vartheta)-\left(\frac{d^{2}}{d z}(\vartheta)\right)^{2}}{\vartheta^{2}} \\
& =\frac{\vartheta^{\prime}}{\vartheta}\left(\frac{\vartheta^{\prime \prime}}{\vartheta^{\prime}}-\frac{\vartheta^{\prime}}{\vartheta}\right)
\end{aligned}
$$


(siehe auch Beispiel 1.12). Offensichtlich hat $\frac{d^{2}}{d z^{2}} \log \vartheta$ in $L$ einen Pol zweiter Ordnung. Den zugehörigen Faktor $c_{-2}$ vor dem Term $\frac{1}{z^{2}}$ in der Laurententwicklung von $\frac{d^{2}}{d z^{2}} \log \vartheta$ in $L$ erhalten wir durch durch:

$$
\begin{aligned}
c_{-2} & =\operatorname{Res}_{L}\left(\frac{\vartheta^{\prime}}{\vartheta}\right)\left(\operatorname{Res}_{L}\left(\frac{\vartheta^{\prime \prime}}{\vartheta^{\prime}}\right)-\operatorname{Res}_{L}\left(\frac{\vartheta^{\prime}}{\vartheta}\right)\right) \\
& =1(0-1) \\
& =-1 .
\end{aligned}
$$

Da das Residuum von $\frac{d^{2}}{d z^{2}} \log \vartheta$ wegen

$$
\begin{aligned}
\operatorname{Res}_{L}\left(\frac{d^{2}}{d z^{2}} \log \vartheta\right) & =\lim _{z \rightarrow 0}\left(\frac{z^{2} \vartheta \vartheta^{\prime \prime}-z^{2}\left(\vartheta^{\prime}\right)^{2}}{\vartheta^{2}}\right)^{\prime} \\
& =\lim _{z \rightarrow 0} \frac{1}{\vartheta^{3}}\left(2 z \vartheta^{2} \vartheta^{\prime \prime}+z^{2} \vartheta \vartheta^{\prime} \vartheta^{\prime \prime}+z^{2} \vartheta^{2} \vartheta^{\prime \prime}-2 z \vartheta\left(\vartheta^{\prime}\right)^{2}\right. \\
& =0
\end{aligned}
$$

verschwindet und der konstante Term $c_{0}$ der Laurententwicklung von der Form

$$
\begin{aligned}
c_{0}= & \lim _{z \rightarrow 0}\left(\frac{d^{2}}{d z^{2}} \log \vartheta+\frac{1}{z^{2}}\right) \\
= & \lim _{z \rightarrow 0}\left(\frac{\left(a_{0} z+a_{1} z^{2}+\ldots\right)\left(2 a_{1}+6 a_{2} z+\ldots\right)-\left(a_{0}+2 a_{1} z+\ldots\right)^{2}}{z^{2}\left(a_{0}+a_{1} z+\ldots\right)^{2}}\right. \\
& \left.+\frac{\left(a_{0}+a_{1} z+\ldots\right)^{2}}{z^{2}\left(a_{0}+a_{1} z+\ldots\right)^{2}}\right) \\
= & \lim _{z \rightarrow 0}\left(\frac{\left(2 a_{0} a_{2}-a_{1}^{2}\right) z^{2}+z^{3}(\ldots)}{a_{0}^{2} z^{2}+z^{3}(\ldots)}\right) \\
= & \frac{2 a_{0} a_{2}-a_{1}^{2}}{a_{0}^{2}}
\end{aligned}
$$

ist, folgt

$$
\frac{d^{2}}{d z^{2}} \log \vartheta=-\frac{1}{z^{2}}+\frac{2 a_{0} a_{2}-a_{1}^{2}}{a_{0}^{2}}+z(\ldots) .
$$

Da die Laurententwicklung von $\operatorname{tr}\left([A, B]^{2}\right)$ gleich

$$
\operatorname{tr}\left([A, B]^{2}\right)=\frac{6}{z^{2}}+c_{0}+z c_{1}+\ldots
$$

ist, ist die Funktion $\operatorname{tr}\left([A, B]^{2}\right)+6 \frac{d^{2}}{d z^{2}} \log \vartheta$ holomorph auf der Jakobischen, also konstant. 


\section{Berechnung des konstanten Terms}

Um die Konstante $g:=\operatorname{tr}\left([A, B]^{2}\right)+6 \frac{d^{2}}{d z^{2}} \log \vartheta \mathrm{zu}$ berechnen, müssen wir zunächst die Laurententwicklungen $\frac{1}{z} A_{0}+A_{1}+z A_{2}+\ldots$ und $\frac{1}{z} B_{0}+B_{1}+z B_{2}+$ ... der Matrizen $A(z)$ und $B(z)$ entlang $\beta$ im Punkt $\mathcal{O}(m) \in \Theta$ berechnen. Die Residuen $A_{0}$ und $B_{0}$ hierzu haben wir in (5.1) und (5.2) bezüglich einer dort fest gewählten Rahmung bestimmt. Weitere Koeffizienten kann man durch das in Schritt S3 beschriebene Verfahren berechnen, wobei jedoch schon die Korrekturterme zu kanonischen Erweiterungen schnell sehr lang werden, beispielhaft siehe hierzu $v_{m-1}(j)$ aus Satz 4.38 und $w_{m}(j)$ aus Satz 4.43. Zur Berechnung des konstanten Terms von $\operatorname{tr}\left([A, B]^{2}\right)$ ist die Berechnung der ersten vier Koeffizienten $A_{0}, \ldots, A_{3}$ bzw. $B_{0}, \ldots, B_{3}$ nötig. Dies folgt aus $\left[A_{0}, B_{0}\right]=0$ und

$$
\begin{aligned}
& \operatorname{tr}\left([A, B]^{2}\right)=\operatorname{tr}\left(\left[\frac{1}{z} A_{0}+A_{1}+z A_{2}+\ldots, \frac{1}{z} B_{0}+B_{1}+z B_{2}+\ldots\right]^{2}\right) \\
& =\frac{1}{z^{2}} \operatorname{tr}\left(\left(\left[A_{0}, B_{1}\right]+\left[A_{1}, B_{0}\right]\right)^{2}\right) \\
& \quad+\frac{1}{z} \operatorname{tr}\left(2\left(\left[A_{0}, B_{1}\right]+\left[A_{1}, B_{0}\right]\right)\left(\left[A_{0}, B_{2}\right]+\left[A_{1}, B_{1}\right]+\left[A_{2}, B_{0}\right]\right)\right) \\
& \quad+\operatorname{tr}\left(2\left(\left[A_{0}, B_{1}\right]+\left[A_{1}, B_{0}\right]\right)\left(\left[A_{0}, B_{3}\right]+\left[A_{1}, B_{2}\right]+\left[A_{2}, B_{1}\right]+\left[A_{3}, B_{0}\right]\right)\right. \\
& \left.\quad+\left(\left[A_{0}, B_{2}\right]+\left[A_{1}, B_{1}\right]+\left[A_{2}, B_{0}\right]\right)^{2}\right)+z \ldots
\end{aligned}
$$

Man beachte hierbei, dass die Matrizen $A(z), B(z)$ nicht die Lax-Paar-Bedingung erfüllen müssen, da a priori keine Rahmung von kovariant konstanten Schnitten gewählt wurde.

Ist der dritte Schritt durchgeführt, sind die Berechnungen in Schritt S4 und S5 leicht: Der konkrete Punkt $\pi_{S}^{*} \mathcal{O}(m-1)=\pi_{S}^{*} \mathcal{O}_{\mathbb{P}^{1}}\left(\frac{1}{2}(k-3)\right) \in \Theta \subset J^{g-1}(S)$ ist bei der Identifizierung $J^{g-1}(S) \simeq J(S)$ der Ursprung, und nach Abschnitt 1.5 eine Thetacharakteristik. Damit folgt insbesondere $\Theta=-\Theta$, d.h. lokal im Ursprung ist die Thetafunktion von der Form

$$
\vartheta(z)=z^{N}\left(a_{0}+a_{2} z^{2}+a_{4} z^{4}+\ldots\right)
$$

mit einem $N \in \mathbb{N}$. Hierbei sind $a_{0}=\frac{1}{N !} \vartheta^{(N)}(0), a_{2}=\frac{1}{(N+2) !} \vartheta^{(N+2)}(0)$, und damit

$$
\begin{aligned}
\frac{d^{2}}{d z^{2}} \log \vartheta(z) & =-\frac{N}{z^{2}}+2 \frac{a_{0} \cdot a_{2}}{a_{0}^{2}}+\ldots \\
& =-\frac{N}{z^{2}}+\frac{2 \vartheta^{(N+2)}(0)}{(N+1)(N+2) \vartheta^{(N)}(0)}+\ldots
\end{aligned}
$$


Wir wissen bereits, dass $\operatorname{tr}\left([A, B]^{2}\right)+6 \frac{d^{2}}{d z^{2}} \log \vartheta(z)$ konstant ist. Damit folgt

$$
\begin{aligned}
N & =\operatorname{tr}\left(\left(\left[A_{0}, B_{1}\right]+\left[A_{1}, B_{0}\right]\right)^{2}\right), \\
0 & =\operatorname{tr}\left(2\left(\left[A_{0}, B_{1}\right]+\left[A_{1}, B_{0}\right]\right)\left(\left[A_{0}, B_{2}\right]+\left[A_{1}, B_{1}\right]+\left[A_{2}, B_{0}\right]\right)\right)
\end{aligned}
$$

und damit

$$
\begin{array}{r}
g=\operatorname{tr}\left(2\left(\left[A_{0}, B_{1}\right]+\left[A_{1}, B_{0}\right]\right)\left(\left[A_{0}, B_{3}\right]+\left[A_{1}, B_{2}\right]+\left[A_{2}, B_{1}\right]+\left[A_{3}, B_{0}\right]\right)\right. \\
\left.+\left(\left[A_{0}, B_{2}\right]+\left[A_{1}, B_{1}\right]+\left[A_{2}, B_{0}\right]\right)^{2}\right)+\frac{2 \vartheta^{(N+2)}(0)}{(N+1)(N+2) \vartheta^{(N)}(0)} .
\end{array}
$$




\title{
Kapitel 7
}

\begin{abstract}
Ausblick
Wir haben in Kapitel 6 beispielhaft die Funktion $\operatorname{tr}\left([A, B]^{2}\right)$ mittels Thetafunktionen ausgedrückt. Zur genauen Beschreibung des Schnittes der Menge $M_{S}$ aller Äquivalenzklassen von Paaren von Matrizen mit fester Spektralkurve $S$ mit der Menge $N_{C}$ aller Äquivalenzklassen von Paaren von Matrizen mit Kommutator $C$ ist die Darstellung weiterer auf $N_{C}$ konstanter Funktionen $f: N_{S} \rightarrow \mathbb{C}$ mittels Thetafunktionen sinnvoll. Interessant wäre hier die für die Berechnung des konstanten Faktors wichtigen Laurententwicklungen der Matrizen $A(z), B(z)$ entlang $\beta$ im Punkt $\mathcal{O}(m)$ explizit zu berechnen und hierzu die Painlevé-Analysis aus Kapitel 3 zu benutzen. Da $\mathcal{O}(m)$ ein singulärer Punkt des Thetadivisors ist, sind $A(z)$ und $B(z)$ formale Laurentlösungen in einer niedrigeren Balance. Durch eine geeignete Eichtransformation kann diese unter Umständen durch endlich viele Koeffizienten der Laurentreihen $A(z), B(z)$ berechnet werden. Eine Einschränkung an die in Frage kommenden niedrigeren Balancen erhält man evtl. bereits aus Dimensionsgründen, indem man $\mathcal{O}(m-1) \in \Theta \subset J^{g-1}(S)$ als Element eines geeigneten Linearsystems $W_{g-1}^{r}=\left\{L \in J^{g-1}(S) \mid h^{0}(S, L) \geq r+1\right\}$ darstellt. Durch die niedrigere Balance wären alle weiteren Koeffizienten der Laurentreihen durch das Gleichungssystem (3.14) bestimmt.
\end{abstract}




\section{Literaturverzeichnis}

[AHH90] Adams, M. R., Harnad, J., Hurtubise, J.: Isospectral Hamiltonian Flows in Finite and Infinite Dimensions. Commun. Math. Phys. 134, S. 555-585, 1990.

[AMV04] Adler, M., van Moerbeke, P., Vanhaecke, P.: Algebraic Integrability, Painlevé Geometry and Lie Algebras. Springer, Berlin, Heidelberg, 2004.

[ACGH85] Arbarello, E., Cornalba, M., Griffiths, P.A., Harris, J.: Geometry of Algebraic Curves, Volume 1. Springer, New York, 1985.

[A89] Artamkin, I. V.: Action of biregular automorphisms of the affine plane on pairs of matrices. Izvestija Math., 33, no. 2, S. 433-439, 1989.

[BBT03] Babelon, O., Bernard, D., Talon, M.: Introduction to Classical Integrable Systems. Cambridge monographs on mathematical physics, Cambridge, 2003.

[B90] Beauville, A.: Jacobiennes des courbes spectrales et systèmes hamiltoniens complètement intégrables. Acta Math. 164, S. 211-235, 1990.

[BL04] Birkenhake, C., Lange, H.: Complex Abelian Varieties. Springer, Berlin, Heidelberg, 2004.

[DRS01] Di Rocco, S., Sommese, A.: Line Bundles for which a projectivized jet bundle is a product. Proc. AMS, Vol. 129, No. 6, S. 1659-1663, 2001.

[E03] Eickhoff, A.: Paare von Matrizen und Linearsysteme auf algebraischen Kurven. Diplomarbeit, 2003.

[F98] Friedman, R.: Algebraic Surfaces and Holomorphic Vector Bundles. Springer, New York, 1998.

[GH78] Griffiths, P., Harris, J.: Principles of Algebraic Geometry. Wiley, New York, 1978. 
[G85] Griffiths, P. A.: Linearizing flows and a cohomological interpretation of Lax equations. Amer. J. of Math. 107, S. 1445-1483, 1985.

[H77] Hartshorne, R.: Algebraic Geometry. Springer, New York, 1977.

[Hi83] Hitchin, N. J.: On the Construction of Monopoles. Commun. Math. Phys. 89, S. 145-190, 1983.

[Hi98] Hitchin, N.: Integrable systems in Riemannian geometry. Surveys in Differential Geometry: Integral Systems, Volume IV, Editoren C. L. Terng, K. Uhlenbeck, International Press, 1998.

[Hi99] Hitchin, N. J., Segal, G. B., Ward, R. S.: Integrable Systems - Twistors, Loop Groups, and Riemann Surfaces. Clarendon Press, Oxford, 1999 .

[M83] Mumford, D.: Tata Lectures on Theta I. Birkhäuser, Boston, 2007 (reprint of 1983 Edition).

[P03] Pidstrygach, V.: On Action of Symplectomorphisms of the Complex Plane on Pairs of Matrices. Fields Institute Comm. 35, S. 157-164, 2003.

[V08] Vanhaecke, P.: Algebraic integrability: A survey. Philos. Transact. A Math Phys Eng Sci Vol 366 no 1867, 2008.

[W73] Wavrik, J. J.: Deforming Cohomology Classes. Trans. Am. Math. Soc. 181, S. 341-350, 1973.

[SD98] Shokurov, V. V., Danilov, V. I.: Algebraic Curves, Algebraic Manifolds and Schemes. Springer, Berlin, Heidelberg, 1998. 


\section{Lebenslauf}

\section{Persönliche Daten}

Annika Eickhoff-Schachtebeck

Blumenbachstraße 2

37075 Göttingen

Tel.: (05 51) 3819290

E-Mail: eickhoff@uni-math.gwdg.de

Geb. am 20.10. 1978 in Bremen

Verheiratet, deutsch

\section{Studium}

seit $04 / 2008$

Wissenschaftliche Mitarbeiterin der Mathematischen Fakultät - Pilotprojekt interdisziplinäre Nebenfachausbildung

04/2007-03/2008 Wissenschaftliche Mitarbeiterin am Mathematischen Institut der Georg-August-Universität

03/2004-02/2007 Stipendiatin des Graduiertenkollegs Gruppen und Geometrie

seit 03/2004 Promotionsstudium bei Prof. Dr. V. Pidstrygach am Mathematischen Institut der Georg - August - Universität Göttingen

01/2004 Diplom

10/1998-01/2004 Studium der Mathematik an der Georg - August - Universität Göttingen; Nebenfach Informatik

10/2000 Vordiplom Mathematik

10/2000-03/2003 studentische Hilfskraft am Mathematischen Institut

\section{Schulbildung}

1998

Abitur

$1991-1998$

Gymnasium Sulingen

1989 - 1991

Orientierungsstufe Kirchdorf

$1985-1989$

Grundschule Kirchdorf 